\title{
Terahertz Channel Propagation Phenomena, Measurement Techniques and Modeling for 6GWireless Communication Applications: a Survey, Open Challenges and Future Research Directions
}

This paper was downloaded from TechRxiv (https://www.techrxiv.org).

\section{LICENSE}

CC BY 4.0

SUBMISSION DATE / POSTED DATE

08-01-2022 / 12-01-2022

\section{CITATION}

Serghiou, Demos; Khalily, Mohsen; Brown, Tim; Tafazolli, Rahim (2022): Terahertz Channel Propagation Phenomena, Measurement Techniques and Modeling for 6GWireless Communication Applications: a Survey, Open Challenges and Future Research Directions. TechRxiv. Preprint.

https://doi.org/10.36227/techrxiv.18092522.v1

$\mathrm{DOI}$

10.36227/techrxiv.18092522.v1 


\title{
Terahertz Channel Propagation Phenomena, Measurement Techniques and Modeling for $6 \mathrm{G}$ Wireless Communication Applications: a Survey, Open Challenges and Future Research Directions
}

\author{
Demos Serghiou, Student Member, IEEE, Mohsen Khalily, Senior Member, IEEE, Tim \\ W.C. Brown, Member, IEEE, Rahim Tafazolli, Senior Member, IEEE
}

\begin{abstract}
The Terahertz (THz) band (0.1-3.0 THz) spans a great portion of the Radio Frequency (RF) spectrum that is mostly unoccupied and unregulated. It is a potential candidate for application in Sixth-Generation (6G) wireless networks as it has the capabilities of satisfying the high data rate and capacity requirements of future wireless communication systems. Profound knowledge of the propagation channel is crucial in communication systems design which nonetheless, is still at its infancy as channel modeling at $\mathrm{THz}$ frequencies has been mostly limited to characterizing fixed Point-to-Point (P2P) scenarios up to $300 \mathrm{GHz}$. Provided the technology matures enough and models adapt to the distinctive characteristics of the THz wave, future wireless communication systems will enable a plethora of new use cases and applications to be realized in addition to delivering higher spectral efficiencies that would ultimately enhance the Quality-of-Service $(Q o S)$ to the end user. In this paper, we provide an insight into $\mathrm{THz}$ channel propagation characteristics, measurement capabilities and modeling methods along with recommendations that will aid in the development of future models in the $\mathrm{THz}$ band. We survey the most recent and important measurement campaigns and modeling efforts found in literature based on the use cases and system requirements identified. Finally, we discuss the challenges and limitations of measurements and modeling at such high frequencies and contemplate the future research outlook toward realizing the $6 \mathrm{G}$ vision.
\end{abstract}

Index Terms-6G, channel measurements, channel modeling, channel sounding, deterministic modeling, diffuse scattering, molecular attenuation, propagation, Radio Frequency (RF), statistical modeling, stochastic modeling, Terahertz (THz), wireless communications.

\section{List OF ABBREVIATIONS}

$6 \mathrm{G}$ Sixth-Generation

AI Artificial Intelligence

AoD Angle of Departure

AoSA Array of Sub-Arrays

BER Bit-Error-Rate

CW Continuous-Wave

FDTD Finite-Difference Time-Domain

FFT Fast Fourier Transform

GBSM Geometry-Based Stochastic Model

Demos Serghiou, Mohsen Khalily, Tim W.C. Brown and Rahim Tafazolli are with the "Institute for Communications, home of the 6G Innovation Centre, University of Surrey, Guildford, Surrey GU2 7XH, UK. (E-mail: d.serghiou@surrey.ac.uk; m.khalily@surrey.ac.uk; t.brown@surrey.ac.uk; r.tafazolli@surrey.ac.uk
GMM

GVD

ICNIRP

IFFT

ISI

iWNSN

LDPC

LoS

$\mathrm{ML}$

MMIC

mmWave

MoM

NGBSM

$\mathrm{RFoF}$

RF

RS

RT

SC

SNR

SV

Tbps

TDS

$\mathrm{THz}$

ToA

UTC-PD

WLAN

$5 \mathrm{G}$

AoA

AP

B-D

B-K

BS

CIR

CTF

D2D

DFT

DSSS

EESS

FDS

FSPL

Gbps

GOA
Gaussian Mixture Model

Group Velocity Dispersion

International Commission on Non-Ionizing Radiation Protection

Inverse Fast Fourier Transform

Inter-Symbol Interference

In vivo Wireless Nano Sensor Network

Low-Density Parity Check

Line-of-Sight

Machine Learning

Monolithic Microwave Integrated Circuit millimeter Wave

Method of Moments

Non-Geometry-Based Stochastic Model

Radio Frequency over Fiber

Radio Frequency

Reed-Solomon

Ray-Tracing

Sliding Correlator

Signal-to-Noise

Saleh-Valenzuela

Tera-bit per second

Time-Domain Spectroscopy

Terahertz

Time of Arrival

Uni-Travelling-Carrier Photo-Diode

Wireless Local Area Network

Fifth-Generation

Angle of Arrival

Access Point

Birth-Death

Beckmann-Kirchhoff

Base Station

Channel Impulse Response

Channel Transfer Function

Device-to-Device

Discrete Fourier Transform

Direct Sequence Spread Spectrum

Earth Exploration Satellite Services

Frequency-Domain Spectroscopy

Free-Space Path Loss

Giga-bit per second

Giant Optical Activity 


$\begin{array}{ll}\text { HEMT } & \text { High Electron Mobility Transistor } \\ \text { HST } & \text { High-Speed Train } \\ \text { InP } & \text { Indium Phosphide } \\ \text { Inter-Satellite } & \text { Link ISL } \\ \text { LO } & \text { Local Oscillator } \\ \text { LTCC } & \text { Low Temperature Co-Fired Ceramic } \\ \text { MIMO } & \text { Multiple-Input Multiple-Output } \\ \text { MPC } & \text { Multi-Path Component } \\ \text { NLOS } & \text { Non-Line-of-Sight } \\ \text { P2P } & \text { Point-to-Point } \\ \text { PN } & \text { Pseudo Noise } \\ \text { QoS } & \text { Quality-of-Service } \\ \text { R-R } & \text { Rayleigh-Rice } \\ \text { RIS } & \text { Reconfigurable Intelligent Surface } \\ \text { RMS } & \text { Root Mean Square } \\ \text { Rx } & \text { Receiver } \\ \text { SI } & \text { International System of Units } \\ \text { SISO } & \text { Single-Input Single-Output } \\ \text { SV } & \text { Support Vector Machine } \\ \text { T2I } & \text { Train-to-Infrastructure } \\ \text { Tx } & \text { Transmitter } \\ \text { UM-MIMO } & \text { Ultra-Massive Multiple-Input Multiple-Output } \\ \text { V2I } & \text { Vehicle-to-Infrastructure } \\ \text { V2X } & \text { Vehicle-to-Everything } \\ & \end{array}$

\section{INTRODUCTION}

$\mathbf{A}^{\mathrm{s}}$ $\mathrm{S}$ the demand for higher data rates is growing exponentially, data rates that exceed 1 Tera-bit per second (Tbps) could be required beyond 2030 [1]. Very high data rates are anticipated in indoor locations where lower costs can be expected for extending wired backbone networks compared to using optical cables while ITU [2] reported that by the year 2030, wireless systems will be as significant as wired cellular systems. A recently published white paper by CISCO estimated that average Fifth-Generation (5G) cellular speeds will reach 575 Mbps by 2023, resulting in a 13-fold increase to the speeds recorded in 2018 [3]. Additionally, Ericsson highlighted the imminent need for capacity as global mobile data traffic is estimated to grow by a factor of 4.5 to reach 226 ExaBytes per month in 2026 [4]. This unprecedented need for higher speeds and larger bandwidths in sight of the currently saturated spectrum below $300 \mathrm{GHz}$ [5] has motivated research in parts of the Radio Frequency (RF) spectrum that have been faintly explored in order to meet the growing needs of the wireless communications industry [6], [7], [8]. A recently published white paper [9] derived within the "6G Flagship" by the university of Oulu in 2019, mentioned that SixthGeneration $(6 \mathrm{G})$ systems should be able to deliver Tbps speeds and that the way to achieve these is through efficient utilization of the $\mathrm{THz}$ spectrum. Nevertheless, initiatives for realizing the $6 \mathrm{G}$ vision have been recently launched such as the "Next G Alliance" project by the USA and "Heat-X" European 6G flagship project [10].

\section{A. Introducing the $\mathrm{THz}$ Band}

The frequency band that lies between the microwave and infrared regions of the electromagnetic spectrum spanning from $100 \mathrm{GHz}$ to $3,000 \mathrm{GHz}$ is termed as the $\mathrm{THz}$ band, with wavelengths ranging from $3 \mathrm{~mm}$ to $0.1 \mathrm{~mm}$ [11]. Recently, the $\mathrm{THz}$ band has received lots of attention as a promising candidate for various applications that can be classified in the fields of wireless cognition [12], [13], [14], sensing [15], [16] Imaging [17], [18], communications [19], [20] and positioning [21], [22], offering advantages such as ultra-fast data transmission [23], increased bandwidth [24] and microsecond latencies [25]. While the $\mathrm{THz}$ wave has proven to be successful in spectroscopy for sensing applications such as material characterization [26], weather characterization [27] and study of astronomical objects [28] due to its frequency selective nature, some first applications identified where the $\mathrm{THz}$ wave could be applied to in 6G networks for wireless communications include Point-to-Point (P2P) scenarios such as kiosk downloads [29], wireless links in data centres [30], intradevice communications [31] and wireless backhaul/fronthaul link extensions [32]. Nonetheless, as the technology continues to mature providing the appropriate beam-steering and tracking capabilities at $\mathrm{THz}$, along with the expected evolution of the semiconductor technology and the ability to enable higher levels of power generation to combat the high losses present in the atmosphere and free space, applications that include mobility and require longer range communication links can also be realized. Applications as such include Wireless Local Area Networks (WLANs), smart rail mobility [33], inter-satellite links (ISL) [34], Vehicle-to-Everything (V2X) communications [35] and Device-to-Device (D2D) communications [36]. Last but not least, $\mathrm{THz}$ has found great interest in future nano-networks within the medical industry for in-body via nano-machine molecular communications [37], [38].

The aforementioned opportunities are ones that arise due to the unique characteristics held by the $\mathrm{THz}$ wave in addition to the largely unoccupied and unregulated region that this end of the spectrum offers. For the development of an efficient communications system with high data rates, deriving the characteristics of the propagation channel by accurate modeling is a necessity. So far, most studies have been limited to frequencies up to the millimeter-wave (mmWave) spectrum (30-300 GHz) due to the lack of high frequency generators and sensitive detectors. At frequencies $>300 \mathrm{GHz}$ propagation models need to consider high path loss, molecular absorption and diffuse particle scattering (due to the short wavelength $<1 \mathrm{~mm}$ ), as the most important phenomena that are very prominent especially as we shift higher up in the $\mathrm{THz}$ frequency spectrum. These characteristics are crucial in the development of successful communications systems in terms of hardware, software and protocols [39].

\section{B. Enabling Technologies for $\mathrm{THz}$ waves}

THz research cannot be pondered as something "new". The first paper ever published [40] dates back to 1893 and it involved THz spectroscopy. However, the term "THz" was not common and instead it was referred to as "obscure" radiation. In the experiments conducted there was a source and detector of $\mathrm{THz}$ radiation and by placing different materials in between, absorption coefficients were measured, much like a lot of 


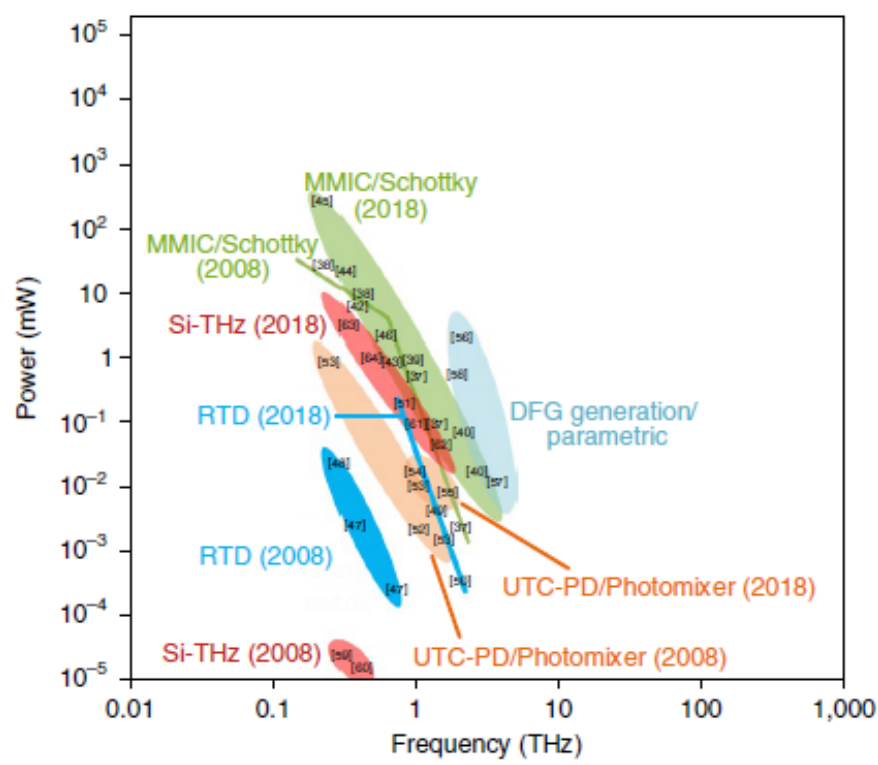

(a)

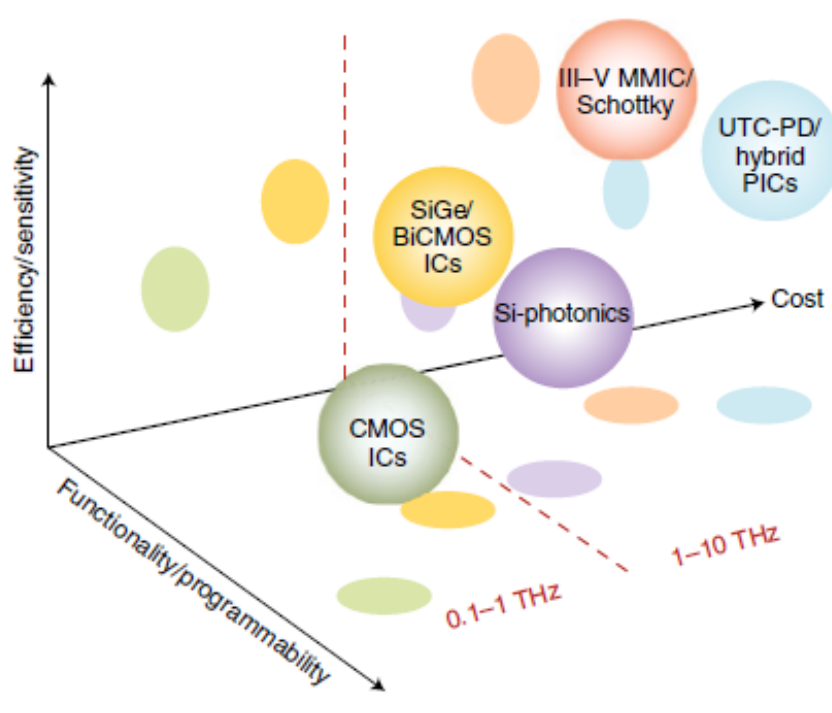

(b)

Fig. 1: Integrated THz systems evolution. (a) Power produced from different sources vs frequency, (b) Efficiency or sensitivity of detectors vs the ability to reconfigure and control these technologies [44].

the measurements conducted nowadays [41], [42], [43]. This shows that while a lot of work has been carried out and a lot of materials have been characterized, there is still a lot of interest and work to be done in basic spectroscopy at THz.

What has happened recently, is that there is a growing realization that the photonics and electronics research coincides and there is an ongoing merging of the electronics and photonics fuse of the world [44]. In recent years there has been a lot of progress in some key metrics and Fig. 1(a) shows one of these metrics in the form of power produced by various different sources vs frequency where e.g., the power produced by silicon sources in 2018 has increased by many orders of magnitude compared to the power levels produced by silicon in 2008, while other examples also show similar sort of improvement. This also shows that the production of integrated systems is possible and that enough power can be generated to do something useful with it. Various techniques are currently being carried out for the development of efficient sources and detectors of $\mathrm{THz}$ radiation [45], [46], [47] to bridge the 'gap' between conventional electronics and optics. Some of which, have made $\mathrm{THz}$ systems affordable and realizable by overcoming existing limitations through novel architectural designs and device structures. On the electronics side, solidstate electronics technologies such as GaAs-based Schottky diodes, Monolithic Microwave Integrated Circuit (MMIC), InP heterojunction bipolar transistors (HBTs), high electron mobility transistors (HEMTs) and Resonant-Tunnelling Diode (RTD) can now enable high power level generation from 100 $\mu \mathrm{W}$ up to the $\mathrm{mW}$ range [48], [49], [50], [51]. This shows an increase of more than double in a decade, especially at frequencies exceeding $1 \mathrm{THz}$. Likewise, various photonic technologies within the $\mathrm{THz}$ band have been developed recently that enable $\mu \mathrm{W}$ Continuous-Wave $(\mathrm{CW})$ power generation including photomixing approaches such as Uni-Travelling-
Carrier Photo-Diode (UTC-PD) and also Quantum Cascade Lasers (QCLs) which are diode lasers capable of operating below $2 \mathrm{THz}$ [52], [53], [54]. DFG/parametric generation within the 1-2 $\mathrm{THz}$ range is also another option at $\mathrm{THz}$ that enables $\mathrm{mW}$ radiated power levels to be obtained [55], [56].

On the other hand, there are many other important metrics besides power that require consideration such as, efficiency or sensitivity of detectors as illustrated in Fig. 1(b). These metrics are important as it is not just a matter of whether it is possible to generate a $\mathrm{THz}$ signal but to also manage to tune and control things such as the direction, spectral content and polarization of the $\mathrm{THz}$ beam [57], [58]. In the spectrum above $1 \mathrm{THz}$ reaching up to $10 \mathrm{THz}$, photonic based systems such as QCLs, Si-photonics and UTC-PD devices are expected to play an important part, especially where tuning of $>1$ $\mathrm{THz}$ is required. It is equally important however, whether these things can be done fast and in a programmable and reconfigurable manner which would require the use of phased arrays [59], [60], [61], [62] that are currently not commercially available. At THz, the beam is required to be very directive in order to combat the high path loss. In a mobile scenario, e.g., when someone walks across the room and the beam needs to follow them, such technology, is known as beam tracking and acquisition, or otherwise, "device discovery" [63], [64]. For the beam to know where to go and to do so quickly enough, phased arrays and processing techniques such as adaptive beamforming are essential [65].

\section{Standardisation Efforts}

A first standard on wireless communications at $300 \mathrm{GHz}$ has been defined by the IEEE $802.15 \mathrm{THz}$ Interest Group known as IEEE 802.15.3d-2017 [66], as an amendment to the IEEE 802.15.3-2016 [67] $60 \mathrm{GHz}$ standard, for operation up to 100 Giga-bits per second (Gbps). The $300 \mathrm{GHz}$ system can utilize 
8 different channel bandwidths between $252-321 \mathrm{GHz}$ as multiples of $2.16 \mathrm{GHz}$ [68], as illustrated in Fig. 2. Both single carrier and on-off modulation schemes are being employed which are combined with Low-Density Parity-Check (LDPC) and Reed-Solomon (RS) codes. Some of the applications identified for this range include P2P scenarios such as kiosk downloads, data centre communications, fronthaul/backhaul, intra-device communications and WLANs.

Spectrum allocation currently ends at $275 \mathrm{GHz}$ for active services, but is not completely unregulated as it is assigned for passive services such as Earth Exploration Satellite Services (EESS) and radio astronomy that gather information based on the resonant frequencies of the molecules and therefore these systems are only receiving and not transmitting [69]. These resonant frequencies are occupied within bands that cover most of the spectrum between $275-1,000 \mathrm{GHz}$, meaning that if we want to allocate these bands for active services and employ $\mathrm{THz}$ communications, this needs to happen by sharing the spectrum [70]. Furthermore, all frequencies between 1,000$3,000 \mathrm{GHz}$ may be also used for both passive and active services [70] though they have not yet been allocated to any service. In regards to the bands between $275-450 \mathrm{GHz}$, the ITU-R has invited its members to conduct sharing studies to see under which conditions and within which frequency bands sharing is possible. At WRC 2019 [69], four contiguous bands were identified between 275 and $450 \mathrm{GHz}(275-296$ $\mathrm{GHz}, 306-313 \mathrm{GHz}, 318-333 \mathrm{GHz}$, and $356-450 \mathrm{GHz}$ ) with large bandwidths i.e.- one of them with $94 \mathrm{GHz}$ available for unrestricted use in total, based on ITU-R studies [71] for land mobile and fixed services without the need to protect EESS. The Horizon 2020-EU-Japan joint project called ThoR has also conducted a sharing study [72] for interference mitigation taking into account the operational characteristic of both passive and active services and its findings are in line with the ITU-R studies. Three bands have been identified (296-306 $\mathrm{GHz}, 313-318 \mathrm{GHz}$ and $333-356 \mathrm{GHz}$ ) in line with bands where proper mitigation is required [73] to avoid harmful interference and allow for coexistence with other applications, while for radio astronomy, on the contrary, proper mitigation techniques are already available.

In conclusion, Frequency bands $>275 \mathrm{GHz}$ are inherently capable for implementing wireless systems with data rates of 100s of Gbps. Feasibility of wireless $\mathrm{THz}$ communication based on current developments and hardware demonstrations has been proven while the first standard is available and so is the spectrum and therefore $\mathrm{THz}$ communication is a real option for employing in future wireless $6 \mathrm{G}$ networks.

\section{Related Works}

To the authors' awareness, no survey paper exists in literature that covers thoroughly $\mathrm{THz}$ channel propagation phenomena, measurements and modeling in the $\mathrm{THz}$ band. Very interesting survey papers that cover channel measurements and modeling can be found in literature that mainly focus on the 5G mmWave bands < $100 \mathrm{GHz}$ [74], [75], [76]; however, propagation characteristics differ from those found at $\mathrm{THz}$ and therefore so do measurement capabilities and modeling

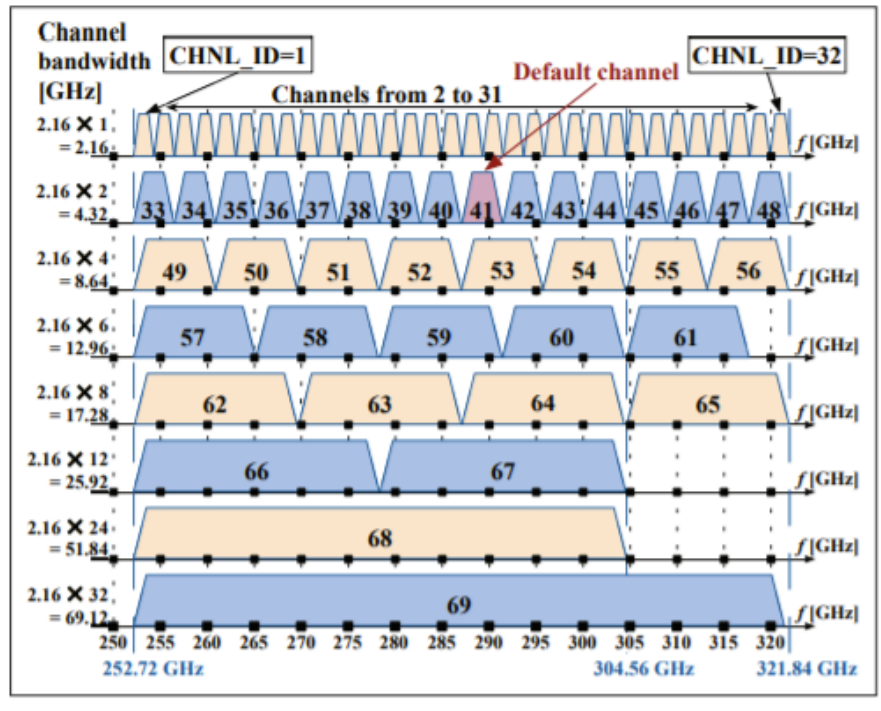

Fig. 2: IEEE 802.15.3d 252-321 GHz bandwidth allocation plan [68].

techniques. Many informative papers can be found that cover many aspects of $\mathrm{THz}$ communications such as applications, technologies and propagation characteristics [77], [78], while some other publications survey channel measurements and modeling for 6G applications [79], [80]; however, they do not focus on $\mathrm{THz}$ alone but cover various aspects of $6 \mathrm{G}$ channel modeling at different frequency ranges. Furthermore, a very useful article found in [81], provides guidelines and considerations for fixed P2P scenarios in the $300 \mathrm{GHz}$ band while in [82] an article can be found that considers modeling methods at $\mathrm{THz}$ along with current issues and future considerations. Moreover, an overview for communications within nano-networks in the $\mathrm{THz}$ band is presented in [83] that covers different aspects of nano-communications and includes a summary of the available methods in modeling for applications such as nano-device in-body communications and on-chip communications.

Much to the information provided on $\mathrm{THz}$ by different articles and surveys mentioned above that cover different aspects of $\mathrm{THz}$ communications related to characteristics, applications, technologies along with studies on the $6 \mathrm{G}$ vision and the role $\mathrm{THz}$ will play; an in-depth survey on measurement and modeling of the THz channel is still not available. In this survey, we aim to narrow this gap in a top-down (holistic) manner, by drawing all our efforts in identifying what has been done so far and what can be further done, as well as what the problems are and how to solve them. Finally, we bring together all of the knowledge we have gathered, as such, to provide a useful insight to the modeling scene in order to motivate and aid in the development of future $6 \mathrm{G}$ wireless communications systems operating at $\mathrm{THz}$ frequencies.

\section{E. Contributions of this Survey}

The aim of this paper is to provide a thorough review of the work found in literature that focuses on measurements and modeling of the propagation channel at $\mathrm{THz}$ that would aid in the development of future models to characterize 
the propagation environment for the deployment of future communications systems. Initially, we describe the different propagation characteristics in terms of specific attenuation due to path loss and precipitation as well as attenuation resulting from various interactions with different materials. We then describe the use cases at $\mathrm{THz}$ and identify what the system requirements for conducting measurements are. Next, we describe the methods that can be employed for channel sounding at $\mathrm{THz}$ as well as channel modeling techniques that can be applied to characterize wave propagation at THz. Furthermore, we survey important works found in literature related to channel characterization for potential $6 \mathrm{G}$ applications. Onward, we present open challenges along with solutions for modeling at $\mathrm{THz}$ frequencies higher than $300 \mathrm{GHz}(\lambda<1$ $\mathrm{mm}$ ), where the current spectrum allocation ends and modeling efforts are still in their early stages. The contributions of this survey are summarized as follows:

- We begin by presenting the different $\mathrm{THz}$ propagation phenomena experienced in free space at $\mathrm{THz}$ and their associated models and describe the unique interactions experienced by the $\mathrm{THz}$ waves in the surrounding environment at this frequency band.

- Secondly, we define and give a short description of the use cases identified and set the system requirements for conducting measurements at $\mathrm{THz}$ based on these use cases. Then, we describe the channel sounding options available that are required for performing measurements at $\mathrm{THz}$, discuss their advantages and disadvantages and discuss how they address the measurement requirements identified.

- Next, we explore the physical and analytical modeling approaches that could be employed at $\mathrm{THz}$ to describe the channel behaviour and discuss which methods are most popular for extracting the propagation channel's characteristics.

- We survey scenario oriented as well as general channel measurement campaigns and modeling efforts found in literature at $\mathrm{THz}$ for static $\mathrm{P} 2 \mathrm{P}$ and dynamic scenarios that include mobility.

- Finally, we present challenges in measurement and modeling at frequencies $>300 \mathrm{GHz}$ and discuss possible solutions and research directions that can contribute to the future modeling scene.

\section{F. Outline}

The structure of this paper is concisely illustrated in Fig. 3. The remainder of this paper is structured as follows; Initially, the main propagation phenomena at $\mathrm{THz}$ and their modeling is presented in Sec. II. Sec. III, introduces the use cases for wireless communications in the $\mathrm{THz}$ band as well as the system requirements for performing meaningful channel measurements and associated performance metrics. In Sec. IV, THz channel sounding methods are introduced which are necessary for conducting measurements based on the use cases identified in order to determine which method is best to be employed depending on each use case's requirements. Channel

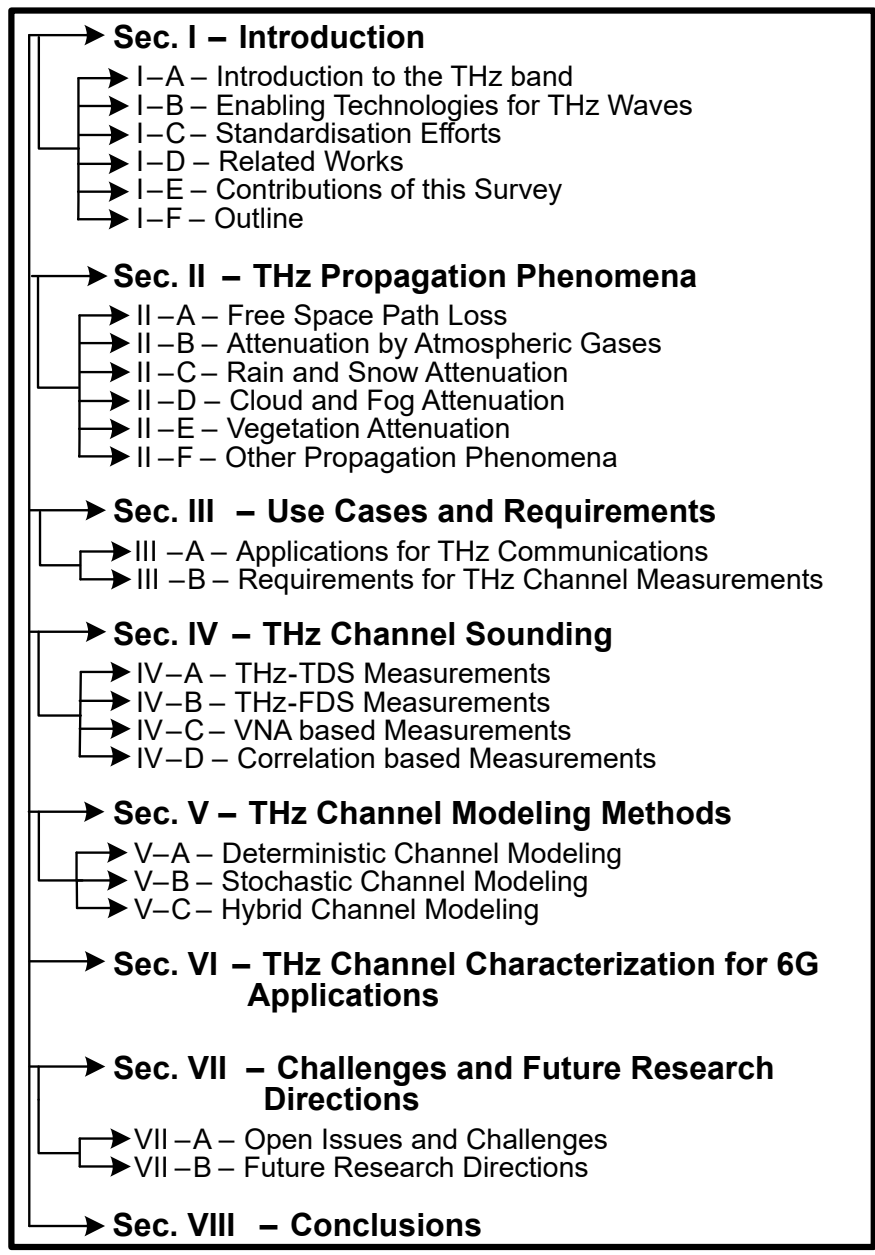

Fig. 3: Structure of this paper.

modeling methods that can be followed for extracting the propagation channel's characteristics in the $\mathrm{THz}$ band categorized as deterministic, stochastic and hybrid are described in Sec. V. In Sec. VI, the most recent and significant modeling efforts for potential static and dynamic $6 \mathrm{G}$ applications as well as for material roughness behavior are reviewed. Open issues and challenges being faced along with solutions and research directions for future modeling works are discussed in Sec. VII. Finally, we conclude the findings of this paper in Sec. VIII.

\section{THz Propagation PhenomenA}

Accurate channel modeling and characterization is required for successful deployment of $\mathrm{THz}$ systems that can comply with the demands of future wireless communication systems. To characterize the $\mathrm{THz}$ channel accurately and efficiently, it is of high importance that the nature of the propagation environment at this frequency range is considered. The THz wave possesses many unique characteristics that make it advantageous in comparison to microwave and mmWave communications such as increased speed and capacity. Propagation phenomena considered in the microwave and mmWave bands include path loss, shadowing, penetration loss and diffraction. In the $\mathrm{THz}$ band, different propagation conditions besides the high path loss become much more pronounced such as molecular 


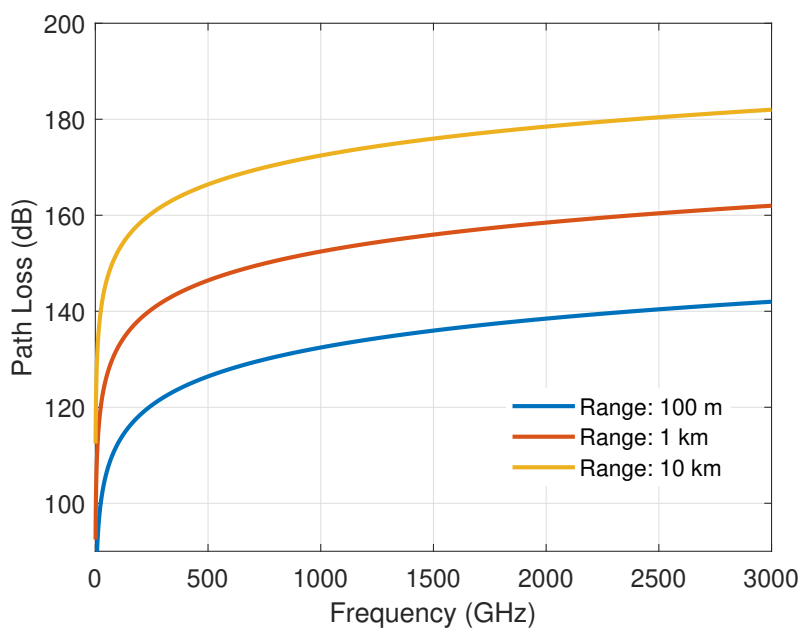

Fig. 4: Free space path loss for the frequency range $1-1,000 \mathrm{GHz}$ with unity gain $\mathrm{Tx}$ and $\mathrm{Rx}$ antennas.

absorption and particle scattering. The effects of rain, fog and cloud also need to be taken into account as in the mmWave bands, while the effects of dust [84] and turbulence [85] are also limiting propagation phenomena, though they are often not as significant.

\section{A. Free Space Path Loss}

Free Space Path Loss (FSPL) is generally defined as inevitable attenuation experienced by a wave propagating in free space between two isotropic antennas [86]. It is described by the Friis transmission formula given by Eq. (1),

$$
P_{\mathrm{r}}(d)=\frac{P_{\mathrm{t}} G_{\mathrm{t}} G_{\mathrm{r}} \lambda^{2}}{(4 \pi)^{2} d^{2}}
$$

where $\lambda$ is the wavelength, $d$ is the distance the wave will travel, with $P_{\mathrm{t}}$ being the transmit power and $G_{t}, G_{r}$ being the Transmitter (Tx) and Receiver (Rx) antenna gains. According to Eq. (1), the received power $P_{\mathrm{r}}(d)$ decreases with the square of the frequency and distance, i.e.- the FSPL attenuation increases proportionally with the decrease in $P_{\mathrm{r}}(d)$. Fig. 4 represents the FSPL for a $\mathrm{P} 2 \mathrm{P}$ link for three different separation distances up to $3 \mathrm{THz}$. Initially, substantial path loss well over $100 \mathrm{~dB}$ is formed at $100 \mathrm{GHz}$, with a $20 \mathrm{~dB}$ per decade increase resulting in an increase by $20 \mathrm{~dB}$ from 0.1 $\mathrm{THz}$ to $1 \mathrm{THz}$ to $10 \mathrm{THz}$ and nearly $10 \mathrm{~dB}$ increase from $1 \mathrm{THz}$ to $3 \mathrm{THz}$. However, in Fig. 4 it is assumed that the antenna gains are equal to unity as calculated from Eq. (2),

$$
G_{t, r}=\frac{4 \pi A_{\mathrm{et}, \mathrm{er}}}{\lambda^{2}}
$$

and the attenuation observed is simply the path loss. If the gain includes information on the aperture area $\left(A_{\mathrm{et}}\right)$ of the Tx $\left(A_{\mathrm{et}}\right)$ and $\mathrm{Rx}\left(A_{\mathrm{er}}\right)$ antennas as in Eq. (2), the term $\lambda^{2}$ from Eq. (1) moves from the numerator to the denomimator in Eq. (3),

$$
P_{r}(d)=\frac{P_{t} A_{\mathrm{et}} A_{\mathrm{er}}}{d^{2} \lambda^{2}}
$$

This means that if the ratios:

$$
\frac{A_{\mathrm{et}}}{\lambda} \text { and } \frac{A_{\mathrm{er}}}{\lambda}
$$

are maintained at any frequency, then the path loss does not change. Therefore the antenna size requirements as we go up in frequency reduce. But at the same time it is generally not possible to increase $A_{\text {et }}$ or $A_{\text {er }}$ at any single frequency beyond a corresponding limit because the aperture efficiency falls consistently in cases such as horn antennas or parabolic dish reflectors. Array antennas as the other solution also have an equivalent limitation in that the increase in number of elements will result in higher feed losses. Also at $\mathrm{THz}$ the closer physical spacing where feeds do not physically size down will cause substantial practical difficulties and further lossy characteristics. Finally, an omnidirectional antenna cannot increase its aperture, which is already nearly as low as an isotropic antenna, by virtue of its design. Therefore based on the above observations, though in theory an increase of $A_{\mathrm{et}}$ and/or $A_{\text {er }}$ will overcome path loss, it is inhibited by physical limitations. To compensate this attenuation, highly directional and high gain antennas are required at the $\mathrm{Tx}$ and $\mathrm{Rx}$ ends which will be much needed, considering the output power decreases with inverse square proportionality to frequency and transmit power will most likely not exceed the order of a few $\mathrm{dBm}$ [87] any time soon.

\section{B. Attenuation by Atmospheric gases}

At $\mathrm{THz}$ the main form of attenuation of the signal is expected due to meteorological attenuation known as atmospheric absorption. The spectrum below $1 \mathrm{THz}$ is considered as the most beneficial and attractive for wireless communications since above that the attenuation is severe and hence, substantially limits the communication distance. When the wavelength of the $\mathrm{THz}$ wave approaches the dimensions of water and oxygen molecules, resonances are generated at selective carrier frequencies resulting in large attenuation peaks that degrade the signal severely playing a major role in link budget calculations [27]. In Fig. 5 we can observe the atmospheric

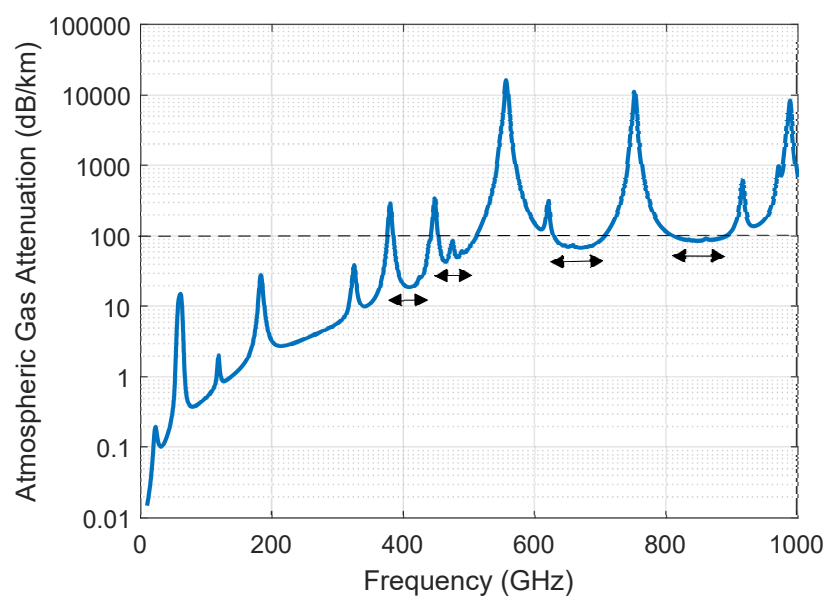

Fig. 5: Atmospheric attenuation in free space for the range 1-1,000 GHz. 
attenuation by water and oxygen molecules over the 1-1,000 $\mathrm{GHz}$ frequency range. This specific attenuation $\gamma$ is calculated by the ITU-R model [88] by aggregating the spectroscopic resonant behaviour of water $\gamma_{0}$ and oxygen molecules $\gamma_{\omega}$ as in Eq. (4),

$$
\gamma=\gamma_{0}+\gamma_{\omega}=0.1820 f N^{\prime \prime}(f) \quad(\mathrm{dB} / \mathrm{km})
$$

where $N^{\prime \prime}(f)$ is the imaginary part of the complex refractive index that can be directly obtained from $\mathrm{THz}$ Time-Domain Spectroscopy (TDS) measurements.

As illustrated in Fig. 5, below $300 \mathrm{GHz}$ the attenuation is minimal reaching up to a few $\mathrm{dB}$ while in the underlined spectral windows between $385-440 \mathrm{GHz}, 453-510 \mathrm{GHz}, 630$ $705 \mathrm{GHz}$ and $815-890 \mathrm{GHz}$ the attenuation ranges up to $100 \mathrm{~dB} / \mathrm{km}$ which still is a substantially massive amount of attenuation, comparable with the attenuation we expect from a Faraday cage. Therefore even with $1 \mathrm{~km}$ we already lose 100 $\mathrm{dB}$ on top of the $\sim 150 \mathrm{~dB}$ in free space. So $250 \mathrm{~dB}$ is far too much attenuation to achieve long distance communications. In addition, different frequency-selective attenuation peaks are observed due to the resonant behavior of molecules in the atmosphere causing severe attenuation levels some of which exceeding $10,000 \mathrm{~dB} / \mathrm{km}$ that would have to be strictly avoided. In the region between 1 to $3 \mathrm{THz}$ the signal undergoes significant attenuation by water and oxygen molecules in the atmosphere and the next atmospheric windows are found between 1.3 and $1.6 \mathrm{THz}$ where the signal is attenuated in the order of a few 100s of dB and may be potentially utilized only for near-field communications $(<0.1 \mathrm{~m})$ [89].

Nonetheless, the applicability of the ITU-R model for the frequency range $450-1,000 \mathrm{GHz}$ was questioned by O'Hara and Grischkowsky [90], as alternative sources that modeled the $>1 \mathrm{THz}$ resonant frequency line contributions to the frequencies $<1 \mathrm{THz}$ by molecular response theory and the absorption continuum using the two-parameter continuum approach, showed more consistency resulting in a closer match to THz-TDS experimental results of total absorption [91], [92]. Moreover, molecular absorption noise is another contributing factor that degrades the signal strength that requires further analysis and appropriate modeling [93].

\section{Rain and Snow Attenuation}

Additional forms of meteorological attenuation that the $\mathrm{THz}$ wave may experience when propagating in an outdoor environment are those of rain and snow [94]. The ITU model provided in [95], describes the rain attenuation, $\gamma_{R}$ in $\mathrm{dB} / \mathrm{km}$ experienced by the $\mathrm{THz}$ wave over the $1-1,000 \mathrm{GHz}$ frequency range given by the power-law relationship in Eq. (5),

$$
\gamma_{R}=k R^{a} \quad(\mathrm{~dB} / \mathrm{km})
$$

calculated by the rain rate $R(\mathrm{~mm} / \mathrm{h})$ where $k$ and $a$ are frequency dependent coefficients derived from scattering calculations at vertical and horizontal polarizations. As the rain droplets' shape and size are in similar order to the wavelength, the propagation losses must be represented as a function of polarization and wavelength. In Fig. 6 the rain attenuation

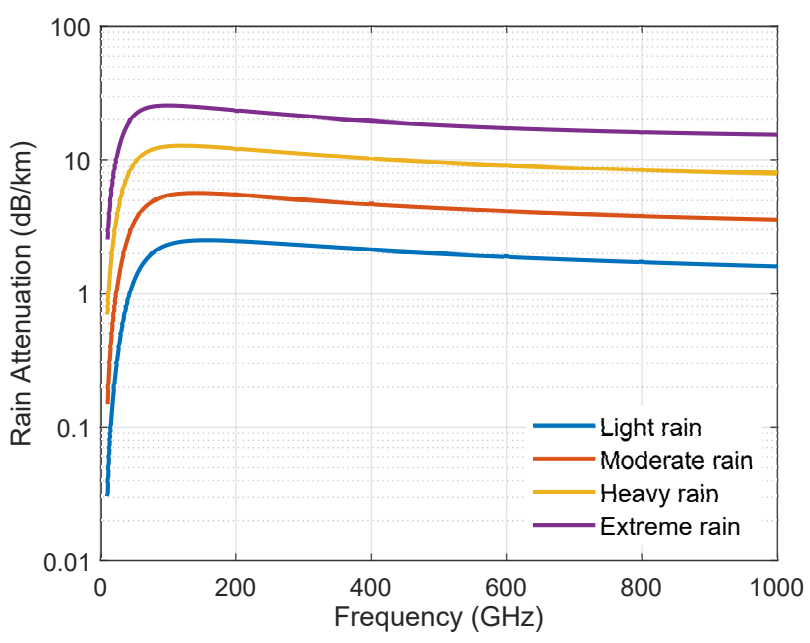

Fig. 6: Rain attenuation for horizontal polarization in the frequency range 1-1,000 GHz.

for horizontal polarization regarded as parallel to ground propagation for the 1-1,000 $\mathrm{GHz}$ frequency range can be observed, representing the worse case of attenuation. Four different scenarios of rain are shown where the rain rate can vary from less than $0.25 \mathrm{~mm} / \mathrm{h}$ for light rain to over $50 \mathrm{~mm} / \mathrm{h}$ for extreme rain conditions. These losses are seen to flatten out around $100 \mathrm{GHz}$, beyond which no additional attenuation is expected within the bands of interest up to $3 \mathrm{THz}$ [96]. The reason for the constant attenuation at higher frequencies is that particle scattering has moved from Rayleigh to Mie scattering [97] due to the shorter wavelength making it more applicable, indicating that particle scattering with pattern similar to that of a dipole radiation pattern (Rayleigh scattering) changes to a more direct scattering pattern with a forward lobe (Mie scattering). These losses may not be as significant as the path losses and atmospheric losses but will have to be considered regardless for accurate channel characterization. With regards to attenuation due to snow, an equivalent ITU propagation model does not exist and the snow particles are merely considered as rain droplets [98]. Therefore, Eq. (5) can also be used to compute the specific attenuation due to snow even though it tends to slightly overemphasize the loss [99].

\section{Cloud and Fog Attenuation}

Another meteorologically induced condition similar to that of rain and snow that can cause fading in an outdoor environment, is that of fog and cloud. Fog and cloud may be considered as the same atmospheric phenomena, that vary only by height above ground. Both phenomena are defined by the ITU model [100] under the assumption that the wave travels solely through a uniform fog or cloud environment. The specific attenuation $\gamma_{c}$ can be computed by Eq. (6),

$$
\gamma_{c}(f, T)=K_{l}(f, T) M \quad(\mathrm{~dB} / \mathrm{km})
$$

where $K_{l}$ is the cloud water specific attenuation coefficient $\left((\mathrm{dB} / \mathrm{km}) /\left(\mathrm{g} / \mathrm{m}^{3}\right)\right)$ found by applying a Rayleigh based doubleDebye scattering model that is used to extract the dielectric 


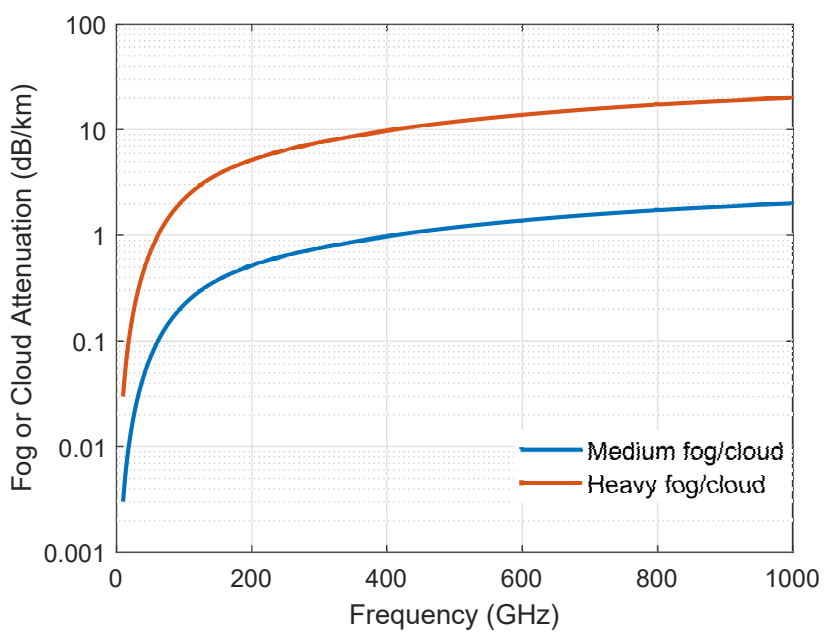

Fig. 7: Fog or cloud specific attenuation for the range 1-1,000 GHz.

permittivity of water and which holds for frequencies between $1-1,000 \mathrm{GHz} . M\left(\mathrm{~g} / \mathrm{m}^{3}\right)$ is the liquid water density in the fog or cloud while $T$ and $f$ are the temperature and carrier frequency, respectively. In Fig. 7 we can see the specific attenuation effects of fog/cloud at a temperature of $20^{\circ} \mathrm{C}$ for $100 \mathrm{GHz}$ carrier frequencies that are typically around 0.05 $\left(\mathrm{g} / \mathrm{m}^{3}\right)$ for medium fog/cloud where the visibility range is in the order of $300 \mathrm{~m}$, and $0.5\left(\mathrm{~g} / \mathrm{m}^{3}\right)$ for heavy fog/cloud where the visibility range drops to $50 \mathrm{~m}$. The losses, similar to those of rain and snow, are much smaller than those of path loss and atmospheric absorption and can be easily compensated for as the attenuation forming at $100 \mathrm{GHz}$ is around $5 \mathrm{~dB}$ with an increase of about $15 \mathrm{~dB}$ from 0.1 to $1 \mathrm{THz}$ for the worst case condition of heavy fog/cloud. Beyond this region and up to 3 $\mathrm{THz}$, the attenuation formed does not vary significantly and increases by just a few dB [96].

\section{E. Vegetation Attenuation}

The presence of vegetation in an outdoor environment plays a significant role in signal attenuation at $\mathrm{THz}$ and may reduce the maximum achievable data rates [101]. High amounts of absorption would be expected due to the high water content while the effects of scattering need consideration as to whether vegetation can contribute to the received signal by Non-Lineof-Sight (NLOS) propagation. Currently, no specific model exists that computes the propagation loss due to vegetation other than the ITU model [102] which holds for frequencies up to $60 \mathrm{GHz}$. This is because the lack of measurement data makes it difficult to develop an appropriate model at $\mathrm{THz}$ frequencies. The THz-TDS technique could be utilized to measure the water content on leaves and the data collected could be later on fed into models that characterize the outdoor propagation environment [103].

\section{F. Other Propagation Phenomena}

Similar to both microwave and mmWave radiation, $\mathrm{THz}$ waves can penetrate through different non-conducting mate- rials such as plastic, clothing and paper [104], [105]. However, strong absorption of the wave, depending on material structure and thickness is extremely possible. Penetration at $\mathrm{THz}$ is limited by the low output power levels and varies by thickness and composition of the material it interacts with [106], [107], [108], while the $\mathrm{THz}$ wave is non-invasive to the human body and is therefore highly susceptible to link blockage in indoor locations (i.e.- when a user is moving across the room) which becomes a major issue when employing communications systems with highly directive antennas. Furthermore, diffraction effects common in the lower bands $<100 \mathrm{GHz}$ that can be described e.g., by the Knife-edge and/or Uniform Theory of Diffraction (UTD) models, are much reduced by comparison at $\mathrm{THz}$ due to the higher directivity, but nonetheless, may be considered as a form of multipath propagation that could contribute to the overall signal strength [109].

Moreover, the scattering behaviour of $\mathrm{THz}$ waves from different surfaces with different roughness levels is unique at $\mathrm{THz}$ owing to the short wavelength and a good understanding of it is required for the development of future channel models. A representation of the different interactions of the $\mathrm{THz}$ waves at a material's surface are illustrated in Fig. 8. EM waves that are directed on a smooth surface should have an equal angle of incidence and reflection (specular reflection). However, when the surface is rather rough, diffuse scattering of the EM waves happens resulting in a loss of intensity in the specular direction. As the $\mathrm{THz}$ wave has a very short wavelength, the scattering of the waves off a rough surface will cause multipath propagation. These rough surfaces can be quantified for $\mathrm{THz}$ frequencies, as having edges that vary between them less than $1 \mathrm{~mm}$ for frequencies $>300 \mathrm{GHz}$. Otherwise, for edges greater than $1 \mathrm{~mm}$ the surface may be considered as smooth. Additionally, multi-layer reflections and reflections within in-homogeneous materials are also another possibility at $\mathrm{THz}$ especially as the permittivity $\left(\epsilon_{r}\right)$ of the material gets closer to unity resulting in additional diffuse components [110]. Other factors that influence the amount of scattering besides wavelength include the size, shape and refractive index of the scatterer [111].

The received impulse response vs time delay for different propagation cases where Line-of-Sight $(\mathrm{LoS})$ is the strongest form of propagation are seen in Fig. 9. Diffuse reflections from rough surfaces cause Multipath Components (MPCs). to propagate randomly and arrive at slightly different times and angles; thus, forming clusters at the peak of the most significant arriving component in contrast to the clear peak expected from a specular reflection off a smooth surface and therefore require careful consideration [112], [113], [41]. Two well known methods that can be employed to predict particle scattering are the Rayleigh-Rice (R-R) [114] and BeckmannKirchhoff (B-K) [115] models that consider smooth and rough material surface interactions, respectively. Temporal broadening is another effect observed at $\mathrm{THz}$ that causes multipath dispersion over time due to the high frequency selectivity as well as fading in the received power levels as also seen in Fig. 9. 


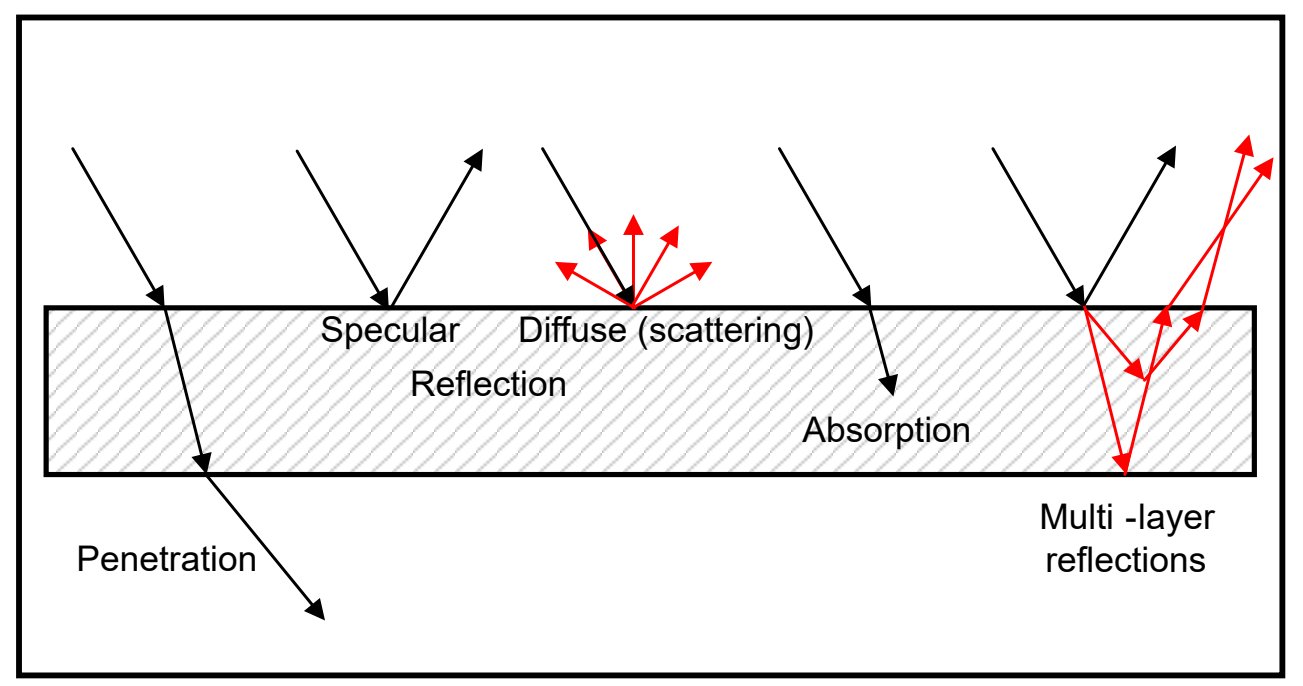

Fig. 8: Material surface interactions in the THz band.

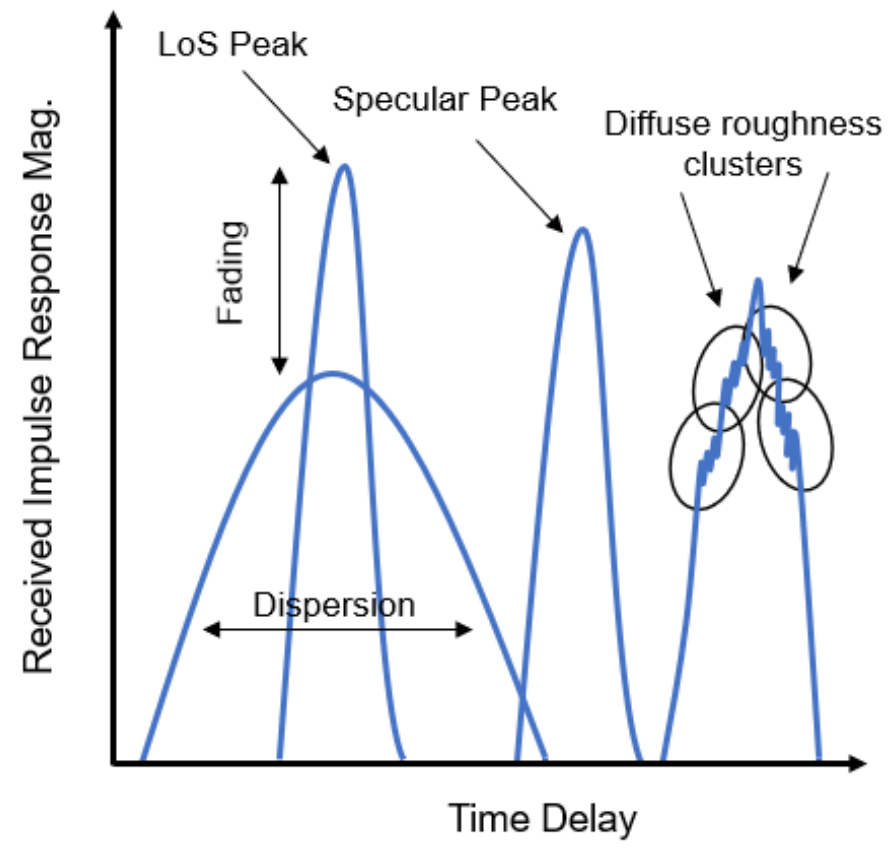

Fig. 9: Received impulse response magnitude vs time delay demonstrating signal arrival and propagation effects experienced in terms of dispersion, fading, specular and diffuse reflection.

\section{USE CASES AND REQUIREMENTS}

For future communication systems deployment, the use cases identified lay a set of requirements and limits for performing meaningful channel measurement campaigns. On that account, in this section the applications for $\mathrm{THz}$ communications are briefly presented. Onward, a general set of system requirements for conducting practical channel measurements are described in order to help in the selection of the appropriate measurement equipment for each of the use cases identified.

\section{A. Applications for $\mathrm{THz}$ Communications}

The governing factor that drives a meaningful measurement campaign is the application scenario under investigation. Generally, all applications identified would benefit from $\mathrm{THz}$ links that would enable high data rate transmissions. Initially, it would be sensible to investigate scenarios that can be characterized by fixed P2P links where the Tx and Rx antennas are considered to be stationary for the whole duration of the measurement such as intra-device, backhaul/fronthaul, data centre communications and kiosk downloads (though in this scenario the Rx side may be subject to minor displacement as the user would have to align the device to the Tx side).

In the foreseeable future, advancing from fixed $\mathrm{P} 2 \mathrm{P}$ scenarios to mobile scenarios, other applications are also envisioned in the $\mathrm{THz}$ band such as communications within $\mathrm{V} 2 \mathrm{X}$ networks, WLANs, D2D communications, ISLs and High Speed Train (HST) communications. These applications would require Multiple-Input Multiple-Output (MIMO) capabilities to achieve extremely high data rates and also large capacities to accommodate a large number of users. Beam steering algorithms for device discovery requiring a high degree of Angle of Arrival (AoA) and Angle of Departure (AoD) estimation as the Tx and Rx will have no a priori knowledge of each other's position, would become essential in order to realize modeling of such applications.

Table I establishes the requirements for channel modeling as to what exactly they are in terms of use cases in relation to expected ranges and environments as well as expected propagation conditions and required antenna characteristics. The use cases identified in the $\mathrm{THz}$ band are briefly described as follows:

- Kiosk/Desktop Downloads: Users downloading a large number of data files from a "vending" type machine to their device such as phone or laptop over a short range in a very short period of time.

- Data Center Networks: Wireless network seamless connectivity between racks in a data center at ultra-high speeds replacing wired connections to simplify data cen- 
TABLE I

Targeted Application Scenarios for THz Communications and Minimum Requirements for Conducting Channel Measurements.

\begin{tabular}{|c|c|c|c|c|}
\hline Application & Propagation Environment & Typical Range & Propagation Characteristics & $\begin{array}{l}\text { Antenna Alignment } \\
\text { Requirements }\end{array}$ \\
\hline Kiosk Downloads & $\begin{array}{l}\text { Indoor; Limited } \\
\text { movement by users }\end{array}$ & $\sim 0.5 \mathrm{~m}$ & LoS, NLoS & $\begin{array}{l}\text { Manual alignment } \\
\text { of Rx by user }\end{array}$ \\
\hline $\begin{array}{l}\text { Data-Center } \\
\text { Networks }\end{array}$ & $\begin{array}{l}\text { Communications between } \\
\text { servers in data center }\end{array}$ & $\sim$ a few 10 s of $\mathrm{m}$ & LoS; NLoS & Manual alignment \\
\hline $\begin{array}{l}\text { Intra-Device } \\
\text { Communication }\end{array}$ & $\begin{array}{l}\text { Communication within } \\
\text { devices; fixed links }\end{array}$ & $\sim$ a few $\mathrm{cm}$ & $\begin{array}{l}\text { LoS; NLoS with significant } \\
\text { multipath contribution }\end{array}$ & Fixed alignment \\
\hline Backhaul/Fronthaul & Fixed wireless link extensions & $\sim 100 \mathrm{~m}$ & $\begin{array}{l}\text { LoS; Atmospheric attenuation; } \\
\text { fog and Rain attenuation; } \\
\text { very high path loss }\end{array}$ & $\begin{array}{l}\text { Highly directive } \\
\text { antennas; fixed alignment }\end{array}$ \\
\hline WLAN & Indoor; mobile users & $\sim 20 \mathrm{~m}$ & LoS; NLoS; scattering & Dynamic beam steering \\
\hline HST Communication & $\begin{array}{l}\text { Communications within trains } \\
\text { and between station } \\
\text { infrastructure and train }\end{array}$ & $<100 \mathrm{~m}$ & $\begin{array}{l}\text { LoS; Directed-NLoS; dynamically } \\
\text { varying conditions; atmospheric } \\
\text { attenuation; high path loss }\end{array}$ & $\begin{array}{l}\text { Dynamic beam steering; } \\
\text { high AoA estimation } \\
\text { accuracy }\end{array}$ \\
\hline $\begin{array}{l}\text { Inter-Satellite } \\
\text { Links }\end{array}$ & $\begin{array}{l}\text { Communications between } \\
\text { satellites in orbit }\end{array}$ & $\sim 1 \mathrm{~km}$ & LoS; satellite drift & dynamic beam steering \\
\hline V2X Communication & $\begin{array}{l}\text { Communications between } \\
\text { vehicles }\end{array}$ & $<100 \mathrm{~m}$ & $\begin{array}{l}\text { LoS; reflected path; dynamically varying } \\
\text { conditions; atmospheric attenuation; } \\
\text { fog and rain attenuation; } \\
\text { high path loss }\end{array}$ & $\begin{array}{l}\text { Dynamic beam steering; } \\
\text { high AoA estimation } \\
\text { accuracy }\end{array}$ \\
\hline D2D Communication & $\begin{array}{l}\text { Communications between } \\
\text { user devices }\end{array}$ & $\sim 1 \mathrm{~m}$ & $\begin{array}{l}\text { LoS; dynamically varying } \\
\text { conditions; molecular attenuation; }\end{array}$ & dynamic beam steering \\
\hline $\begin{array}{l}\text { Nano-Network } \\
\text { Communication }\end{array}$ & $\begin{array}{l}\text { Nano-device communication } \\
\text { within nano-networks; in-vivo; }\end{array}$ & $\sim$ a few $\mathrm{cm}$ & Tissue penetration loss; scattering & Dynamic beam steering \\
\hline
\end{tabular}

ter deployment and maintenance in terms of installation costs and hardware configuration/reconfiguration and also to improve cooling.

- Intra-Device Communications: Chip-to-Chip, board-toboard as well as on-board and on-chip wireless $\mathrm{THz}$ links within devices that eliminate the need for wired connections, thus reserving more space within the device and reducing energy consumption.

- Wireless Backhaul/Fronthaul: Fronthaul THz links to extend usually beyond where the fibre can't reach to give broadband access to the end user. Backhaul $\mathrm{THz}$ links used to connect the base station to the core network.

- WLAN: Wireless connectivity that would require connections between various access points by replacing fibreoptic cables and thus enabling higher data rates.

- HST Communications: High data rate communications between train and infrastructure as well as intra-wagon communications that will enable seamless connectivity, safer travel and lower costs.

- Inter-Satellite Links: Communication links between satellites that lie outside the earth's atmosphere that do not suffer by molecular attenuation. Unlike the optical bands at $\mathrm{THz}$, strict beam alignment requirements are less imposing and enough to maintain a good alignment as satellites shift away from their orbits. Along with the large bandwidth that is currently not available in the lower bands, the $\mathrm{THz}$ band is a good candidate to be employed for future ISLs.

- V2X Communications: THz Communications within vehicular networks that can support a wide range of scenarios involving vehicles, infrastructure and pedestrians offering benefits such as enhanced user experience, improved road safety and air quality.

- D2D Communications: D2D communications have surfaced as a major technology for enhancing spectrum utilization in the next generation of cellular networks. D2D exploits the close proximity of various devices in order to share resources efficiently so that higher data rates, reduced latency and higher levels of system capacity can be achieved.

- Nano-Networks: Nano-machine enabled communications such as in-vivo communications within sub-mm sized regions inside the human body that are otherwise not accessible by any other medical device.

\section{B. Requirements for $\mathrm{THz}$ Channel Measurements}

a) Bandwidth: While the spectrum is vastly available above $300 \mathrm{GHz}$ in the $\mathrm{THz}$ regime, channels at multiples of $2.16 \mathrm{GHz}$ have been introduced that allow for contiguous bandwidths of a few 10 s of $\mathrm{GHz}$ targeting data rates of 100 Gbps. More specifically, as per [66], data rates around 100 Gbps can be achieved with an 8-PSK modulation scheme with about $50 \mathrm{GHz}$ bandwidth. Higher order modulation schemes should be employed to give higher data rates, such as 64QAM that is able to provide data rates $>200 \mathrm{Gbps}$ for the same bandwidth $(\sim 50 \mathrm{GHz})$ [66]. To satisfy the capacity requirements and achieve high data rates, channels composed of many 10 s of $\mathrm{GHz}$ of contiguous spectrum that can be freely allocated for active services with high order modulation and sophisticated coding schemes will be required.

b) Antenna Directivity/Alignment: Side-lobe suppression and spatial filtering are the main requirements for antennas operating at $\mathrm{THz}$, something that cannot be provided by omni-directional antennas used in the lower frequency bands. Therefore, highly directive antennas with high gain are required which are very much necessary to combat the high path loss and large atmospheric absorption effects of the highly frequency selective $\mathrm{THz}$ channels and also provide strong enough signal components that would be detected by the receivers. The antenna selected should be easily rotated and 
measurements should adapt to each scenario by interchanging the antennas for accurate channel characterization. Additionally, with the use of multiple antennas in the form of phased arrays and in conjunction with technological advancements in beam-forming and beam-steering along with improvements in AoA estimation algorithms, high directionality and high gain could be acquired that would be used to achieve the desired data rates.

c) Link Distance: The measurement distance separating the Tx and Rx side of the communications channel determines the extent to which accurate channel measurements at $\mathrm{THz}$ frequencies can be conducted. Indoor measurements require Tx-Rx separation distances of a few meters while in outdoor scenarios 10s and even 100s of meters of measurement distances will be required where various effects would have to be catered for such as a large number of reflections from buildings, vegetation and fading from meteorologically induced attenuation. Cabling is a largely negative factor that currently affects measurements over long distances. A Radio Frequency Over Fiber (RFoF) extension for frequency channel sounding was developed for the first time in [116] to extend the Tx-Rx channel measurement distance above $100 \mathrm{~m}$ and was successfully demonstrated in an outdoor environment. An important factor here is the system limitation when a $\mathrm{THz}$ radio link has several $\mathrm{GHz}$ of system bandwidth. The standard definition for noise figure is derived from the added noise by the receiver, $N_{\text {Rec }}$ :

$$
F=1+\frac{N_{\mathrm{Rec}}}{N_{0}}=1+\frac{T_{\mathrm{Rec}}}{T_{0}}
$$

where $N_{0}$ is the the noise power added by the receiver and both have corresponding noise temperatures from use of kTB. Therefore taking an ambient temperature $T_{0}$ of $290 \mathrm{~K}$, this calculates to form $-84 \mathrm{dBm}$ for $1 \mathrm{GHz}$ bandwidth then up to $-74 \mathrm{dBm}$ for $10 \mathrm{GHz}$ bandwidth. This noise floor would increase by up to another $10 \mathrm{~dB}$ based on the noise figure at the receiver defined as:

$$
\text { Noise Figure }=10 \log _{10}\left(1+\frac{T_{\mathrm{Rec}}}{T_{0}}\right)
$$

where $T_{\mathrm{Rec}}$ is the temperature of the added noise from the receiver. So what is measured over fibre might exceed what is achieveable actually by a $\mathrm{THz}$ system.

d) Dynamic Range: The dynamic range of the equipment used for measurements is very important in determining the signal strength of different MPCs. A high order of diffuse reflections coming from the very short wavelength of the $\mathrm{THz}$ wave interacting with irregularities on surfaces similar in magnitude to the wavelength and indeed significant contributions of specular reflection components resulting from the highly directive beam are expected. For that reason, the dynamic range of the measuring equipment will help in identifying these resulting components that will be very helpful to determine the effect they have on the overall received signal strength. Therefore, the noise floor must be at a low enough level to achieve a high dynamic range, quantified as the difference between the strongest power component received and noise level. e) Doppler Sampling Rate: The Doppler effect is where the propagating waves' frequency changes based on the velocity of a moving object in relation to the sources vicinity. E.g., a user moving across the room and blocking the signal transmission in an indoor scenario or equivalently a vehicle moving away from a stationary source located on another vehicle in a nearby lane in an urban scenario. The maximum Doppler shift, $f_{\text {d,max }}$ can be described by Eq. (9),

$$
f_{\mathrm{d}, \max }=\frac{v_{\max }}{\lambda}=f_{\mathrm{c}} \cdot \frac{v_{\text {max }}}{c_{0}}
$$

where $v_{\max }$ is the maximum velocity of the moving object, $\lambda$ is the wavelength, $f_{\mathrm{c}}$ is the carrier frequency and $c_{0}$ is the speed of light.

For a user moving across the room with a velocity of 6 $\mathrm{km} / \mathrm{h}$ with a wavelength of $1 \mathrm{~mm}$ at $300 \mathrm{GHz}$, that would result in a $f_{\mathrm{d} \text {,max }}$ of $1.6 \mathrm{kHz}$. Similarly, for a vehicle travelling at a velocity of $60 \mathrm{~km} / \mathrm{h}$ for the same wavelength that would give us a $f_{\mathrm{d} \text {,max }}$ of $16.7 \mathrm{kHz}$. This parameter is extremely important to consider when measuring the dynamic behavior of the channel to obtain its time-variant characteristics while its effects will be much more significant at $\mathrm{THz}$ compared to the lower frequency bands. In order to avoid aliasing effects where signals become indistinguishable from one another and cannot be reconstructed at the $\mathrm{Rx}$, the Doppler spectra must be sampled sufficiently fast at a rate $\geqslant 2 f_{\mathrm{d} \text {,max }}$ in accordance with the Nyquist-Shannon sampling Theorem [117], [118].

f) Multi-Path Resolution: High timing accuracy that will enable accurate characterization with high delay resolutions is expected with equipment capable of capturing the characteristics of the rich multipath environment when measuring over large bandwidths where clusters of MPCs would be easily detected by their Time of Arrival (ToA). As an example, in [119] measurements over an ultra-wide frequency range of $250 \mathrm{GHz}$ were conducted using a VNA that allowed for a very high delay resolution of 4 picoseconds given 1600 data points. Clear and similar in shape LoS and specular peaks could be seen, while distorted peaks with different clusters of distinguishable MPCs appeared as a consequence of diffuse particle scattering from rough surfaces of various test materials' placed intentionally as reflectors in the propagating environment. High precision rotational [120] and translational [121] stages are also required to capture multipath propagation within the angular and spatial domains when performing such measurements.

g) Other Requirements: The choice of channel sounder to be used largely depends on the given application scenario e.g., whether its for material characterization, a fixed P2P communication scenario or a mobile scenario and what other requirements the targeted application has, such as bandwidth, speed, accuracy etc. What is a common need, is that of a relatively cheap and compact channel sounder that is easy to transport and one where cabling between the channel sounder and $\mathrm{Tx} / \mathrm{Rx}$ would be as less complicated as possible to avoid calibration issues that are very common when performing these measurements. Furthermore, for short range scenarios, the channel sounder itself when large in size may act as an undesired reflector. In addition, to allow for accurate 


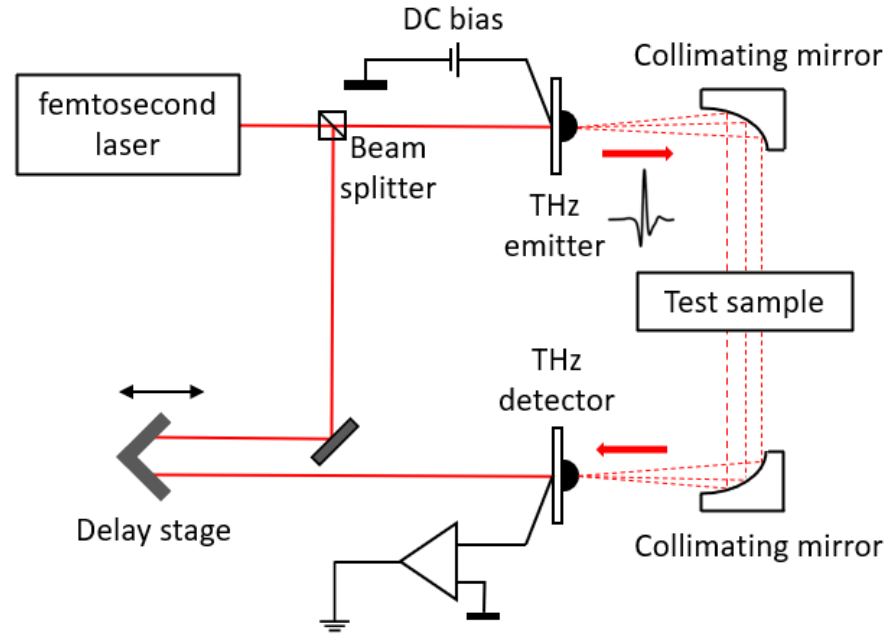

(a)

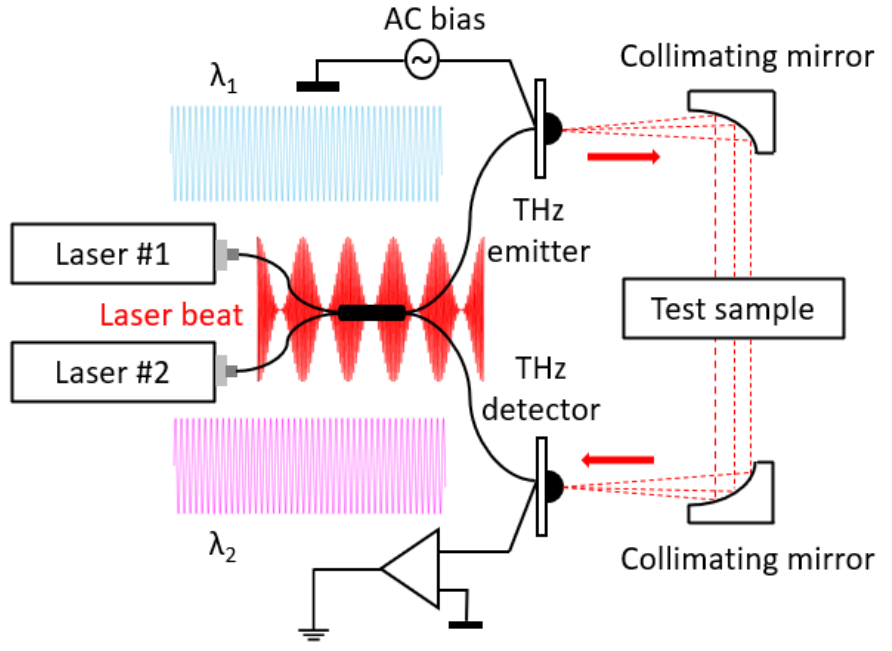

(b)

Fig. 10: Experimental setup for THz signal generation. (a) THz-TDS, (b) THz-FDS

$\mathrm{THz}$ channel measurements the channel sounding system has to be characterized itself in order to establish metrological traceability to the International System of units (SI) [122].

\section{THz Channel Sounding Techniques}

As a requirement to communications system design, profound knowledge of the propagation environment is essential. In order to develop future channel models, channel measurements are the first and most important step that require state of the art sounding equipment and correct methods to be available. $\mathrm{THz}$ measurements have been mainly conducted by three distinct channel sounding methods. These include the well known THz-TDS [123], Vector Network Analyser (VNA) [124] and Sliding Correlator (SC) channel sounder [125], while THz-FDS (Frequency-Domain Spectroscopy) [126] is another very promising channel sounding option. In this section, the performance of these channel sounding methods is described and compared with measurement requirements that may vary depending on the application scenario.

\section{A. THz-TDS Measurements}

THz-TDS [123], as depicted by Fig. 10(a) is a channel sounding technique where material properties are being probed by ultra-short light pulses. These pulses are converted to the $\mathrm{THz}$ range using photo-conductive switches (GaAs or InGaAs/InP) irradiated by femtosecond lasers at the source of the Tx and the received optical impulses detected at the Rx are converted to an electrical signal [127]. The received time domain signal is then processed by Fast Fourier Transform (FFT) to the frequency domain as a much more efficient implementation of the Discrete Fourier Transform (DFT) used to extract its spectral content. Moreover, since the received signal is complex (i.e.- containing both amplitude and phase information) it can be used to calculate the complex refractive index whose real part is used for measuring material thicknesses and the imaginary part used for calculating resonant absorption and extracting conductivity directly without the need for additional modeling [123]. THz-TDS has the advantage of large bandwidth measurements where bandwidths up to $10 \mathrm{THz}$ have been achieved [128] allowing for a wide range of spectral fingerprints of different dielectric materials to be identified, supplemented by a very good dynamic range $(\sim 100$ $\mathrm{dB}$ ) and tunable timing resolution that is achieved by adjusting the distance the two pulses will propagate; hence, enabling paths to be resolved within femtoseconds [123]. Additionally, since this is a pulsed technique, it allows for time-gating that can increase the Signal-to-Noise (SNR) ratio which normally decreases with thickness or number of layers, and allows for content extraction between multi-layered structures of sub$\mathrm{mm}$ thicknesses; thus, proving to be excellent at measuring the scattering parameters and electrical properties of various material samples [129].

\section{B. THz-FDS Measurements}

THz-FDS [126] is a channel sounding technique that employs $\mathrm{CW} \mathrm{THz}$ radiation to probe the material properties. The THz-FDS measurement setup is illustrated in Fig. 10(b). It is a technique that utilizes optical heterodyne detection in highbandwidth photo-conductors that involves optical signal and oscillating wave mixing where the combined product gives an electrical signal at the difference frequency of the two lasers. It offers an excellent dynamic range $(\sim 100 \mathrm{~dB})$ for large bandwidth measurements $(\sim 6 \mathrm{THz})$. Two lasers at adjacent frequencies are used to illuminate the photo-mixer and the applied bias creates a photo-current that is modulated at the beat frequency. This allows for the antenna to transmit the $\mathrm{THz}$ wave and produce a monochromatic beam. Furthermore, by tuning the lasers, the $\mathrm{THz}$ wavelength can be adjusted. The main advantage of THz-FDS compared to THz-TDS is that is offers the user a very high frequency resolution (10 $\mathrm{MHz}$ typ.) at a user determined range or fixed frequency and a comparatively lower cost [126].

Nevertheless, the THz-FDS technique as with THz-TDS is limited by its very small output power and large size and 
cannot be used for long range channel characterisation and for a wider range of scenarios. Furthermore, this technique cannot capture the dynamic behaviour of the channel such as Doppler spread due to moving scatterers blocking the direct path, as it requires the channel to be stationary for the whole duration of the measurement, which may take several minutes.

\section{VNA Measurements}

The main technological advancements related to capturing the characteristics of the propagation channel are namely, VNA [124] and SC [130] channel sounders. The VNA, whose operation is more well known works by sending a stimulus signal from the Tx to the $\mathrm{Rx}$ and processing the received signal to obtain the channel properties. It measures the frequency response, which is essentially a measure of the phase and magnitude of the received signal by processing sequentially a great number of Channel Transfer Functions (CTFs) as a function of increasing frequency within the band of interest and then aggregating them together to generate a broadband CTF, i.e.- the $S_{21}$ parameter which is a measure of the signal coming out of port 2 in relation to the signal entering port 1 of the VNA and is used to characterize distance-varying measurements. Onward, using Inverse Fast Fourier Transform (IFFT) the Channel Impulse Responses (CIRs) are generated as a function of time delay similar to those shown earlier in Fig. 9, section II. A VNA can provide full synchronization between the received and transmitted signal enabling individual calibration and coherent averaging over long times for the suppression of noise because of the large number of narrowband measurements [131]; hence, making it ideal for narrowband channel characterization. To extend the frequency range of the VNA to the $\mathrm{THz}$ range, frequency extensions are employed for the up conversion of the signal [132], [133].

A VNA based measurement setup is depicted in Fig. 11. The setup consists of a VNA connected at the Tx and $\mathrm{Rx}$ sides to frequency extender modules which in turn are connected to a waveguide and horn antenna that must be fixed precisely to avoid causing any signal reflections back to the frequency extender. To realize such a setup practically and enable an accurate measurement campaign and account for system errors in transmission and also due to source mismatches, system directivity and reflection tracking [119], the VNA/ extender heads require lengthy calibration techniques,

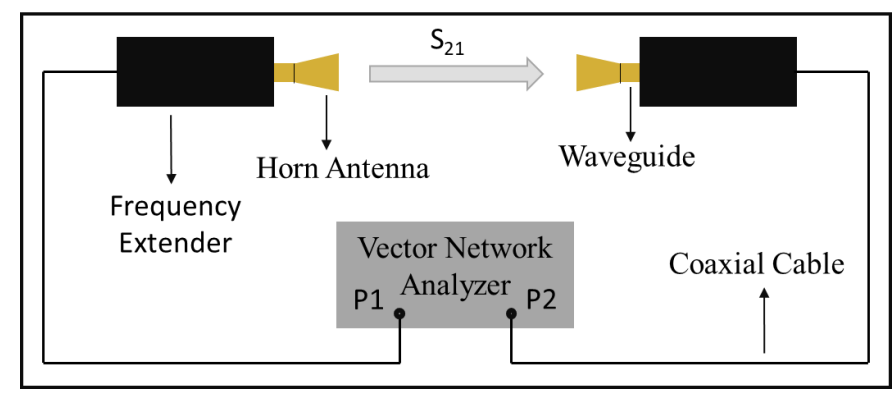

Fig. 11: Schematic of a VNA based measurement setup with frequency extenders employed for up-conversion of the signal. e.g., Short, Offset-short, Load and Thru (SOLT) [134]. One of the key limitations related to measurements using VNAs is that the frequency extenders have to be connected via physical connections to maintain phase synchronization; thus, the measurements are constrained in terms of Tx-Rx separation distance while at these short distances the VNA must be placed somewhere near the Tx and Rx ends therefore, acting as an unwanted reflector and scatterer. Furthermore, the cabled connections between $\mathrm{Tx}$ and $\mathrm{Rx}$ are very sensitive and may affect the calibration if they are moved around. Another key limitation of the VNA is the ability to measure the propagation characteristics over ultra-wide frequency ranges of a timevariant channel influenced by moving scatterers i.e.- humans that cause blockage and scattering of the signal. In such complex cases, the applicability of VNAs is no longer viable since they take too long to perform a full frequency sweep surpassing the channel coherence time [130], and therefore not allowing for dynamic channel variations such as Doppler shifts to be captured in order to analyze the effects of link blockage. Nevertheless a VNA can provide an excellent dynamic range $(\sim 120 \mathrm{~dB})$ [134] and a very high delay resolution [110] allowing for a very large number of MPCs to be resolved over significantly broad bandwidths; Nonetheless, the bandwidth is directly dependent upon the selected waveguide interface [135] used for Single-Input Single-Output (SISO) measurements. Frequency extender modules that are commercially available can operate between $50 \mathrm{GHz}$ to $1.1 \mathrm{THz}$ with varying bandwidths that reach up to $350 \mathrm{GHz}$ in the higher end [136].

\section{Sliding Correlator Measurements}

The most popular channel sounding method that can yield real-time results is the SC based measurement method that utilizes the Direct Sequence Spread Spectrum (DSSS) technique that was first introduced by "Cox" of the Bell Laboratories [137]. The SC based time-domain channel sounding method, operates by transmitting a broadband Pseudo-Noise (PN) sequence $S_{\mathrm{Tx}}(t)$ along the channel $h(t)$ from the Tx to the $\mathrm{Rx}$ as a stimulus signal resulting to the received signal $b(t)$ given by Eq. (10),

$$
b(t)=\int h(\zeta) \cdot S_{\mathrm{Tx}}(\tau-\zeta) d \zeta
$$

and calculated by convolution of the propagating signal with the time-varying channel. At the receiving side, crosscorrelation of the received signal with an identical but delayed by $\tau$ complex conjugate of the PN sequence $S_{\mathrm{Rx}}^{*}(t-\tau)$ takes place as in Eq. (11),

$$
\begin{aligned}
E\{b(t) & \left.S_{\mathrm{Tx}}^{*}(t-\tau) d \zeta\right\} \\
& =E\left\{\int h(\zeta) S_{\mathrm{Tx}}(t-\zeta) S_{\mathrm{Rx}}^{*}(t-\tau) d \zeta\right\} \\
& =\int h(\zeta) S(\tau-\zeta) d \zeta .
\end{aligned}
$$

Given the auto-correlation function $P(\tau-\zeta)$ of the PN sequence can be described by a Dirac-impulse shape which 
TABLE II

COMPARISON OF THE Four DifFerent CHANNEL SOUNDing TECHNiQues.

\begin{tabular}{llll}
\hline \hline & TDS / FDS & VNA & SC \\
\hline Measurement Domain & Time / Frequency & Frequency & Time \\
Bandwidth & $>5 \mathrm{THz}$ & a few 100s of GHz & $8 \mathrm{GHz}$ \\
Dynamic range & $\sim 100 \mathrm{~dB}$ & $\sim 120 \mathrm{~dB}$ & $\sim 30 \mathrm{~dB}$ \\
Speed & milliseconds to 1 min / several mins & several minutes per sweep & $1,000 \mathrm{~s}$ of CIRs generated per sec \\
Frequency resolution & $10 \mathrm{GHz}$ typ. / 10 MHz typ. & $10 \mathrm{MHz}$ typ. & - \\
Real-time & Yes / No & No & Yes \\
Range & $<1 \mathrm{~m}$ & a few 10s of m & a few 10s of m \\
Spectral selectivity & No / Yes & Yes & No \\
\hline \multirow{2}{*}{ Use Cases/Applications } & Intra-device links, scattering properties; & Kiosk downloads, WLAN, & V2X communications, HST communications, \\
& molecular attenuation & outdoor, backhaul/fronthaul & dynamic link blockage, D2D communications \\
\hline \hline
\end{tabular}

then equals to the CIR, a maximum length sequence ( $m$ sequence) can therefore be used as a PN sequence to be transmitted as a reference signal in order to approximate the Dirac-impulse shaped function [118], [138], [139]. The advantage of this channel sounding method is that it can capture all the frequency points at the same time so that it is not as slow as frequency scanning measurements conducted using a VNA.

In Fig. 12 a simplified schematic of a SC based channel sounder is presented. The pseudo-noise sequence is mixed up to achieve the targeted frequency range before modulation, known as the baseband. In order to estimate the complex CIRs in the baseband, the $\mathrm{Tx}$ and Rx sides need to be in synchronization to achieve phase recovery at the $\mathrm{Rx}$ side, something that is achieved by the clock source that is used to drive the Local Oscillator (LO) on the Tx and Rx sides with a high degree of precision. Hence, an SC can measure a dynamic channel but not a mobile channel due to the clock source.

Time-domain SC operates at much higher speeds and allows for dynamic variations of the channel to be captured caused i.e.- by moving scatterers. However, strong thermal noise consequently may affect the precision in obtaining the results due to the lack of measurement bandwidth that is limited by the semiconductor technology [130]. SC channel sounding in the mmWave bands below $100 \mathrm{GHz}$ with realtime capabilities that allow for fast electronic steering of the $\mathrm{THz}$ beam through the use of phased antenna arrays has been recently demonstrated [140], [141], [142]. Nevertheless, the first SC channel sounders at $\mathrm{THz}$ have also been introduced at $140 \mathrm{GHz}$ [106] with $4 \mathrm{GHz}$ bandwidth and at $300 \mathrm{GHz}$ [125] at an ultra-broad bandwidth of $8 \mathrm{GHz}$, with the latter being

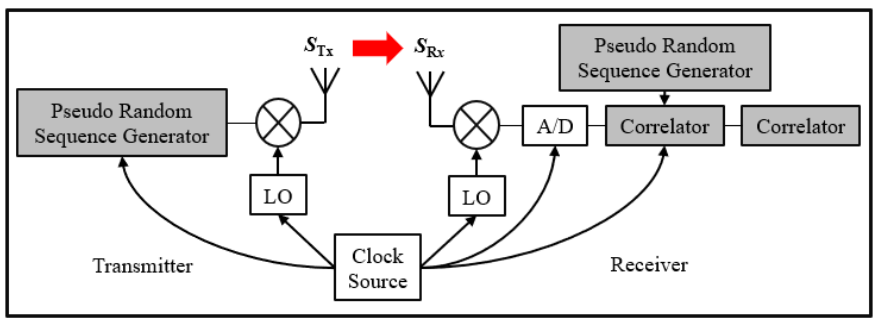

Fig. 12: Schematic of a Correlation based channel sounder. able to capture 17590 CIRs per second, while also having $4 \times 4$ MIMO capabilities.

\section{E. Summary}

Table II summarizes and compares the capabilities of the four main channel sounding techniques described in this section and names a few of the applications that these methods would work best for. Ideally channel sounders in the future would maximise these capabilities that are imperative for achieving better characterization of the propagation channel and would adapt to a wider range of scenarios and potential applications in the $\mathrm{THz}$ band.

\section{THz Channel Modeling Methods}

Deriving the channel characteristics by appropriate modeling is the next step after conducting measurements in communication systems design. Current modeling approaches can be either described as deterministic or stochastic in the form of Geometry-Based Stochastic Models (GBSMs) and NonGeometry-Based Stochastic Models (NGSMs). Deterministic models such as Ray-Tracing (RT) [143] require precise modeling of the propagating environment in terms of positioning of the antennas, geometry and properties of the scatterers present, in a mapped $3 \mathrm{D}$ environment to determine the power received at each point in space. Deterministic models, even though they can accurately predict the characteristics of the propagating environment, suffer from high computational complexity and large simulation times. On the other hand, stochastic models, are ones that use measurement data to give a statistical description of the propagation channel. NGBSMs are those where the scatterers are not modeled explicitly and the propagation channel is considered as a number of delay taps where by deriving probability density functions through extensive empirical data collection we can establish the channel model. In GBSMs probability distributions are used to describe the properties of scatterers and then fundamental laws of wave propagation are used to characterize the propagation process. The delay taps in NGBSMs are replaced by clusters in GBSMs that represent a collection of MPCs with similar properties where the clusters are distinguishable from one another. In the following sub-sections we describe these models in more detail as to their applicability at $\mathrm{THz}$ frequencies. 


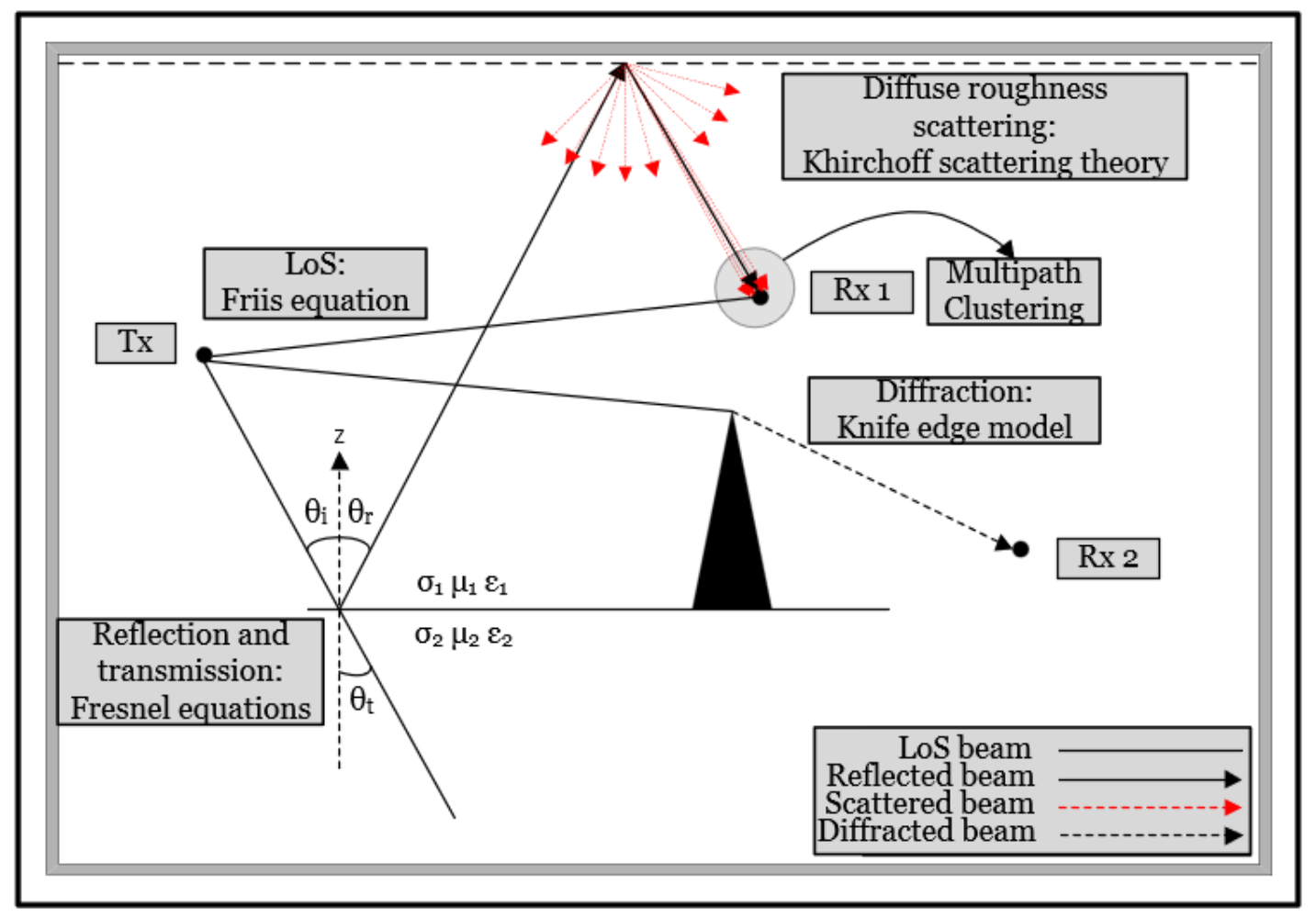

Fig. 13: The RT method for deterministic channel modeling.

\section{A. Deterministic Channel Modeling}

Deterministic channel models are ones that use the electromagnetic laws of wave propagation to determine the signal power received at a particular location in a 3D mapped environment. Deterministic channel modeling approaches in the $\mathrm{THz}$ band involve accurate geometric modeling of the wave propagation environment by EM theory and require exact knowledge of the boundary conditions. Accurate positions of the $\mathrm{Tx}$ and $\mathrm{Rx}$ antennas along with geographical positions and properties of different material present such as shape and dielectric constant are needed to ensure the measured and expected results are in conjunction. The most popular and common deterministic channel modeling technique that has been employed successfully is known as RT [144].

$\mathrm{RT}$ in the THz spectrum can be employed to model various scenarios related to wireless communication scenarios within e.g., indoor office rooms, data centres and nano-networks. It is a method by which rays are launched at regular time intervals and are detected based on propagation in free space where effects such as reflection, scattering from rough surfaces as well as edge diffraction and clustering are accounted for as illustrated in Fig. 13. Advanced acceleration and high efficiency 3D RT techniques need to be developed that can increase the simulator's performance and which consider the propagation environment characteristics precisely. Detailed geographic and geometric knowledge of the propagation environment in each scenario is essential for accurate modeling. In order to accurately employ RT to estimate the channel characteristics, the model requires parameters such as angle of incidence to the reflective surface, propagation distance and the complex permittivity of the materials considered as reflectors in the model. Depending on the complexity of the model higher accuracy in the estimated results may be achieved. For this, the scattering behaviour considering a high order of diffuse reflections needs to be accounted for, which requires knowledge of how well each material absorbs energy and how rough the material is. Moreover, when RT needs to be applied to simulate practical MIMO systems where high data rates can be achieved, the computational complexity increases massively as the simulation needs to account besides scattering, communication between multiple antennas over ultrawide frequency bands [145]. Furthermore, it is a challenge to be able to analyse the time-varying properties of the channel. This accounts for switching between LoS and NLoS scenarios, varying the $\mathrm{Tx}$ and $\mathrm{Rx}$ locations as well as varying conditions in the atmosphere and of the surrounding scatterers in the propagation environment.

On the other hand, Finite-Difference Time-Domain (FDTD) [146] and Method of Moments (MoM) are numerical methods that can be used to account for back-scattering of the rays. They are methods that can solve Maxwell's equations precisely by integral (FDTD) and differential (MoM) equation analysis. They can provide accurate estimates of spatial distributions when detailed geometric knowledge is required in terms of roughness of materials, shape etc. However, The amount of computational resources required for simulations are extensively large and the processes are time consuming therefore limiting the size of the environment to be modeled. This is because the solution to Maxwell's equations requires a set of points spaced out in the order of a wavelength so that a 
numerical solution to the equations can be found. This means that the computational complexity of the problem increases massively as the frequency and amount of scatterers increases. Both the FDTD and MoM approaches mentioned are very attractive and may be combined with RT to provide a more reliable estimation method. Although; combining the two models together may be quite challenging since they are devised using different tools and assumptions. Furthermore, large-scale and small-scale interactions are tightly coupled and thus the hybrid model developed needs to consider mutual coupling before deployment. For the development of an efficient model at $\mathrm{THz}$, additional parameters need to be considered such as polarization, indoor-to-outdoor propagation and channel variations caused by moving scatterers [147].

\section{B. Stochastic Channel Modeling}

Stochastic channel models are ones that use empirical data to provide a statistical analysis of the channel by a spatiotemporal approach. These models are based on measurement campaigns and require much less complexity in terms of computing resource requirements compared to deterministic models. Although, measurement time to obtain good accuracy in the results is a limiting factor. Due to the higher order frequencies and larger bandwidths, wideband channels in the $\mathrm{THz}$ regime require multipaths to be resolved in the time domain. Narrow-band fading models such as Rayleigh and Ricean may therefore no longer be required and a statistical model that can generate the CIRs needs to be developed. This would require us to obtain the spatio-temporal characteristics of the propagation channel which are described in more detail as follows:

- Spatial Characteristics: Spatial parameters can be extracted from a Power Angle Profile (PAP) that involves statistical distributions of the different multipaths in the form of power received over AoA and AoD. PAP measurements can be conducted by varying a highly directional antenna in azimuth and elevation using high precision rotational and translational stages and recording the power received with the advantage that no phase information is required to steer the beam in the wanted direction in contrast to electronic beam-steering [148]. Misalignment of the antennas and signal arriving from different angles will cause power losses, and therefore it is important to know how much power is received at a given point in time. Root Mean Square (RMS) angular spread is a spatial parameter that can be extracted from the PAP which describes the power arrival dispersed over the angles of incidence the antenna is being varied. Despite the high directivity of antennas at $\mathrm{THz}$, this angular spread may prove to be significant especially at longer distances, i.e.- in an urban environment, where spreads in the order of $+/-40^{\circ}$ where recorded when measured using a $30^{\circ}$ beamwidth horn antenna [116].

- Temporal Characteristics: The temporal behavior of the channel can be described in the form of a Power Delay Profile (PDP) which records power received over ToA of the different arriving MPCs where each component

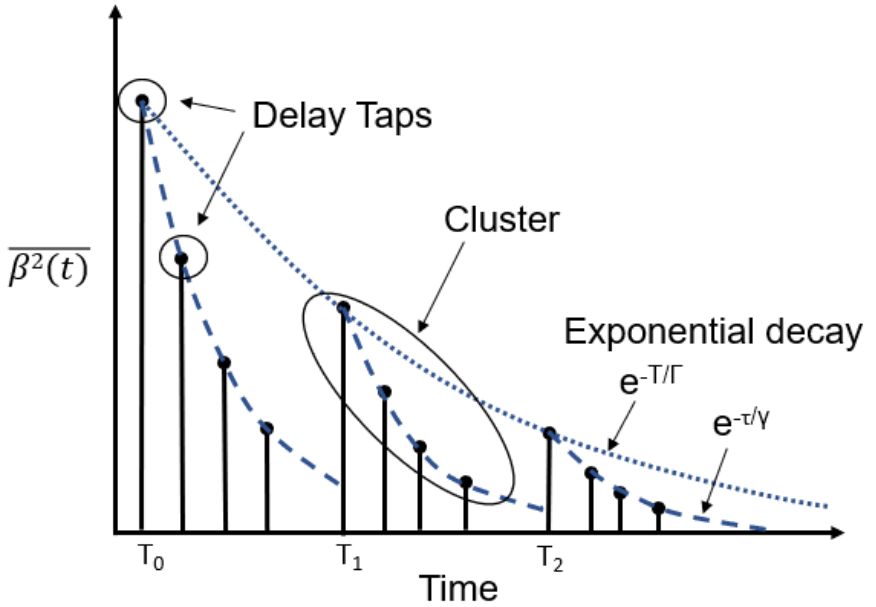

Fig. 14: SV model representing exponential decay of clusters and rays over time.

represents a single channel delay tap. It is important that the channel sounding method employed offers a high delay resolution to separate the different multipaths over very small periods of time. Time dispersion parameters such as RMS delay spread and coherence bandwidth are parameters that can be extracted from the PDP and are used to provide information for the design of transmitters and receivers that can overcome the effects of fading. At $\mathrm{THz}$ frequencies, very small delay spreads would be observed, where up to 4 ns delay spreads were measured experimentally in an indoor environment at $300 \mathrm{GHz}$ [149].

Many factors need to be considered in the future for accurate channel modeling. Clustering behaviour is observed when the arriving MPCs or otherwise intra-cluster components have similar distributions in terms of ToA and AoA/AoD. Saleh and Valenzuela [150] proposed a widely acceptable NGBSM known as the Saleh-Valenzuela (SV) model that provides a cluster based impulse response based on reflections from different surfaces where MPCs are modeled as delay taps. It assumes that MPCs vary uniformly in phase and have powers that are Rayleigh distributed and which decay exponentially based on a Poisson distributed ToA of MPCs and clusters. Fig. 14 shows the SV model's impulse response that represents the mean square power $\left(\overline{\beta_{2}(t)}\right)$ over time where clusters are seen to decay with a rate of $\Gamma$ with cluster arrival time $T_{l}$ for $l=0,1,2 \ldots$ and MPCs are seen to decay with a rate of $\gamma$ with MPC arrival rate $\tau_{k l}$, where $k$ is the MPC arrival time for $k=0,1,2 \ldots$ within the $l^{\text {th }}$ cluster. Extensions of the SV model have already been developed at lower frequencies [151], [152], [153] to account for the cluster AoA in addition to its temporal behavior. Thus, PDP and PAP measurements may be used as a basis from which the SV model can be applied to at $\mathrm{THz}$ to fit and verify the resultant clustering behavior [154], [155], [156].

In order to further analyze the multipath behavior within individual clusters, a second order profile for exponentially distributed multipaths such as the Gaussian mixture model 


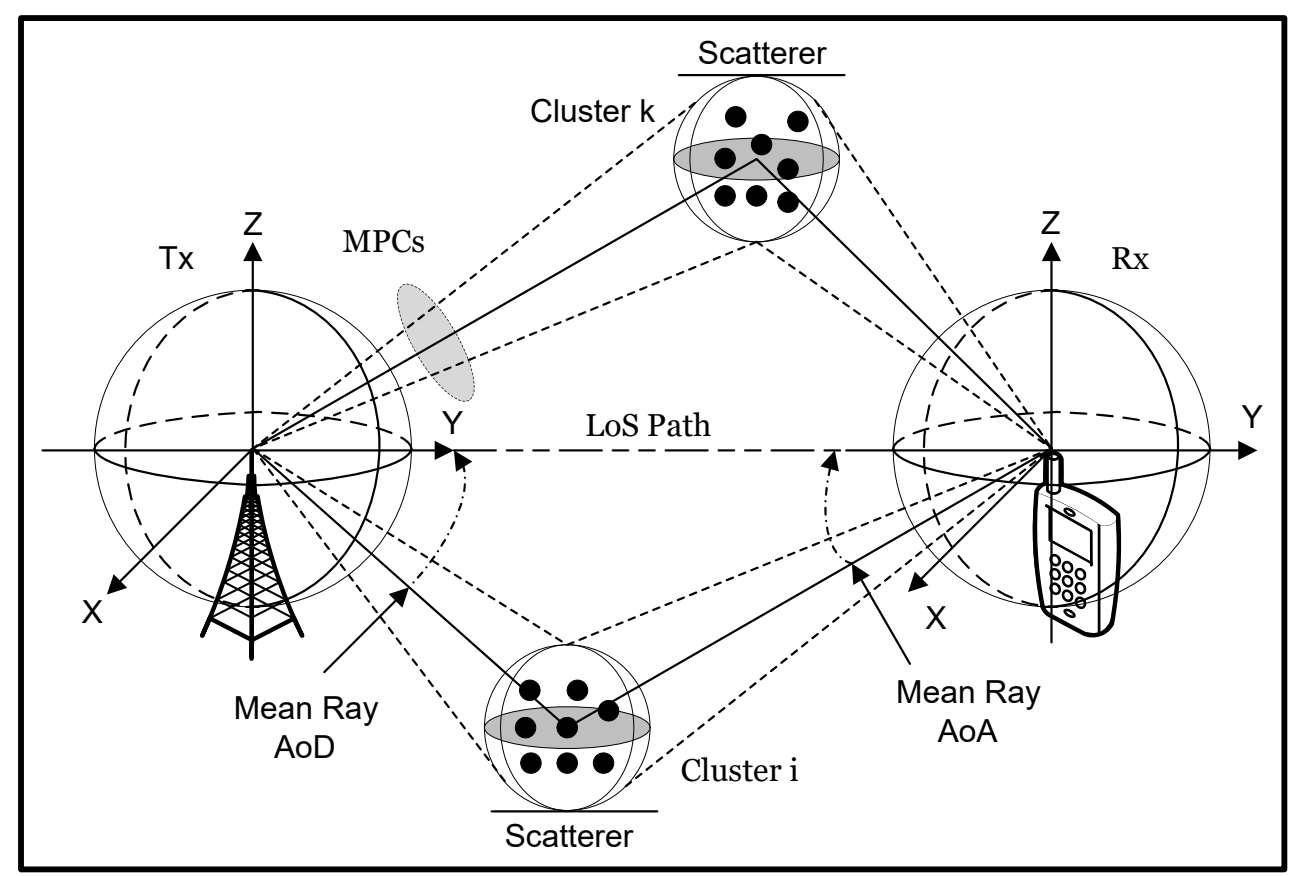

Fig. 15: The cluster oriented GBSM.

(GMM) [157] [158] which is a cluster oriented GBSM that can be adopted for approximating AoA/AoD and identifying similarities between the different MPCs within a cluster or otherwise inter-cluster components. A generalized representation of a cluster based GBSM is depicted in Fig. 15 that shows a collection of rays being launched from the Tx with similar AoD, reflected at the surface of a scatterer and detected at the $\mathrm{Rx}$ at some AoA and ToA. As per Eq. (12),

$$
\sum_{n}^{M}\left(\alpha_{\mathrm{Tx}}^{I}\right)\left(\alpha_{\mathrm{Rx}}^{2}\right)\left(e^{\mathrm{j} \theta_{n}}\right)\left(e^{\mathrm{j} \phi_{n}}\right)\left(e^{\mathrm{j} \omega_{\alpha}}\right) \quad[\bar{\Phi}]
$$

these individual rays $n$ are summed up to form a single cluster with a phase at the Tx $\left(e^{\mathrm{j} \theta_{n}}\right)$ and mean $\operatorname{AoD}$ of $\theta$, and phase at the $\operatorname{Rx}\left(e^{\mathrm{j} \phi_{n}}\right)$ with mean AoA of $\phi$, where the total number of rays is denoted as $M$. Then by computing the gain of each individual ray at the Tx $\left(\alpha_{\mathrm{Tx}}^{1}\right)$ and $\mathrm{Rx}\left(\alpha_{\mathrm{Rx}}^{2}\right)$ sides as a function of polarization $\bar{\Phi}$, a single complex number is formed representing a single delay tap of the multipath channel, where the real and imaginary parts are Gaussian distributed and therefore the single complex number formed is Rayleigh distributed. The Doppler effect could also be accounted for by adding the term $e^{\mathrm{j} \omega_{\alpha}}$ to the equation where $\omega$ relates to the Doppler frequency.

\section{Hybrid Channel Modeling}

Now what seems to be happening at $\mathrm{THz}$, is that a hybrid modeling approach, that is, a combination of both deterministic and stochastic methods is currently being preferred. To capture the received power at every point in space and time, channel sounding at $\mathrm{THz}$ often makes use of mechanically translational and rotational antennas; though, taking a very large sample of measurement data is often extremely time consuming and modeling the environment in a stochastic manner with high levels of accuracy is extremely difficult. Therefore, in order to extend the sampling intervals within a 3D environment to further extract the statistical behavior of the channel, software simulators such as RT are employed which by themselves often appear rather simplistic due to lack of knowledge of the propagating environment and limited computational resources. When empirical measurements are conducted, there are often different components and effects that appear which are not captured by RT simulators such as dense MPCs. Thus, by feeding the RT simulator a set of empirical data, it can be then calibrated by replicating this data through extensive simulations to enable a much larger data set as an extension to the limited data sets collected by measurements. Therefore, statistical modeling with stochastic scatterer placement and RT Hybrid channel modeling could inevitably satisfy all the propagation conditions and may prove to be a promising method for the development of future models that combine the advantages held by deterministic and empirical models so that a balance can be obtained between accuracy of the results held by deterministic models and reduced complexity held by stochastic models generated from empirical data. Nonetheless, the hybrid approach still fails to address the problem of complexity due to the need of extensive measurement and long simulation times. Therefore, it should be pointed out, that this hybrid approach could benefit from machine learning to reduce the extent of measurements required [159].

\section{THz CHANNEL CHARACTERIZATION FOR 6G APPLICATIONS}

In this section we highlight the main channel measurements and modeling contributions found in literature for describing the stationary as well as dynamic behavior of the $\mathrm{THz}$ channel. 
We categorize these models based on the application scenarios identified in section III for future $\mathrm{THz}$ communications.

1) Kiosk/Desktop Downloads: Kiosk downloading may be considered as a short range communication scenario where file exchange between user devices such as smartphones or PCs and "vending" type machines can be pondered as prospective use cases with the potential to provide the so called "information showers" and achieve extremely high data rates targeting $100 \mathrm{Gbps}$ [160]. Propagation in such use cases may be assumed to be LoS over a short distance $(<1 \mathrm{~m})$ with the uncertainty of user equipment antenna misalignment where the user would have to essentially point the receiving device towards the transmitting end. The presence of a front cover window would induce specular reflection while the Tx side would have to be equipped with a high gain antenna to compensate for propagation losses as antennas placed i.e.within a smartphone would have their signal strength reduced significantly because of the smartphone casing. Song $e t$ al. [161] presented a kiosk data downloading model based on a MMIC for the transmitter with Indium Phosphide (InP) based High Electron Mobility Transistor (HEMT) semiconductor technology along with concepts and considerations for small packaging for receiver modules based on the Low Temperature Co-Fired Ceramic (LTCC) technologies, where 20 Gbps error free transmission was achieved over $1 \mathrm{~m}$. This Kiosk downloading system was further prototyped for operation at 300 $\mathrm{GHz}$ and tested experimentally and a link budget plan was also presented [162]. He et al. [29], [163] presented a stochastic channel model for 3 different Kiosk downloading scenarios at $300 \mathrm{GHz}$ where up to $6^{\text {th }}$ order reflections were considered and channel parameters were obtained based on measurement and calibrated ray-tracing results. Salhi et al. [164] investigated broadband angular channel characteristics by conducting measurements for 5 different frequency bands up to 321 $\mathrm{GHz}$ for a short range indoor desktop download scenario that can be considered as a very similar use case to the kiosk download scenario. Recently, short range characterization was extended to carrier frequencies of $625 \mathrm{GHz}$ with $250 \mathrm{GHz}$ bandwidth to measure the channel properties in the the ultrawideband [119], [110]. It was found that specular components from nearby scatterers are significant while reflections from various materials can induce diffuse components that form clusters due to the high frequency selectivity at $\mathrm{THz}$, which nonetheless, is extremely unpredictable.

2) Data Center: A data center can be referred to as the infrastructure within a building that contains racks with servers with links connecting together the different racks as well as the servers within these racks [165]. A potential application of the $\mathrm{THz}$ wave because of the high bandwidth availability is wireless data center communications where the cabled connections would be replaced by ultra-fast and high data rate wireless links. This would offer the advantages of flexibility and low costs as well as easier to maintain environmental conditions provided by cooling technologies for equipment operation. Cheng et al. [166] presented a THz MIMO channel sounding measurement campaign in a data centre like environment at $300-312 \mathrm{GHz}$ where LoS and Directed-NLoS (DNLoS) propagation were considered and the data was modeled in the form of delay dispersion, shadowing gain and path loss. Similarly, an amplitude fading analysis and Doppler modeling was considered for a $4 \times 4$ system, and a $2 \mathrm{D}$ geometrical model based on moving scatterers was also presented and validated with measurement data [167]. Characterization of different propagation phenomena for a wireless data centre was considered in [30] while PAP and PDP measurements in an actual data centre were presented in [168] for various $\mathrm{P} 2 \mathrm{P}$ scenarios. In [169] a stochastic channel model was developed based on the ray tracing approach that was used to derive the statistical properties of the channel where up to 21 different paths with their associated delays and losses were considered in the simulation along with dispersion effects caused by phase changes from small movement of the Tx and Rx. An evaluation of the propagation channel in a $\mathrm{THz}$ wireless data centre was presented in [170] where the effects of humidity were considered. Finally, in [156] a cluster based model as a modification of the SV model was presented where the effects of cooling on signal transmission were accounted for along with path loss and shadowing effects. The model was further validated with measurement data.

3) Intra-Device: The continuous rise in data transfer within modern day devices where communications between components, different layers of motherboards and within on-chip networks are expected, has lead to complicated layout structures, reduced processor space and high energy level consumption [171]. As such, high data rate intra-device $\mathrm{THz}$ wireless communication links may prove to be a solution. The propagation environment to be considered for channel modeling is very small, in the order of a few $\mathrm{cm}$ due to the casing of the device. The antenna requirements may be more flexible with less directive characteristics but communications are expected to rely not only on LoS propagation but also by significant multipath scattering contributions within the small enclosure by NLoS propagation. Examples of intra-device communications include board-to-board, chip-to-chip [172], [31] as well as in-chip communications [173], [174], [175]. Fu et al. [31], presented measurements conducted within a metal casing at $300 \mathrm{GHz}$ with the results showing that the received power is sensitive to the heights of the Tx and Rx sides. In [176] characterization of transmission scenarios for $\mathrm{THz}$ intradevice communications was presented. Measurements were conducted in the frequency range between $270-320 \mathrm{GHz}$ with LoS and NLoS scenarios being considered where the narrow propagation environment was found to induce less multipath contribution while the impact of printed circuit boards was found to play a role in NLoS communication but has not been yet investigated in more detail. Data rates of $0.95 \mathrm{Tbps}$ were achieved by full-wave simulations in [177] for wireless on-chip communications that interconnect cores in a multiprocessor. In [178] the effects of multipath propagation on the link budget due to multi-layer housing structures for on-chip communications were studied and may prove to be beneficial if careful chip layer structure is considered.

4) Backhaul/Fronthaul: Wireless backhaul/fronthaul links are potential use cases for future $\mathrm{THz}$ communications with LoS as the main propagation characteristic. A backhaul link is a fibre based or wireless link that connects the base station 
to the core network and is in the $\mathrm{km}$ range. Current wireless backhaul links are not sufficient for future capacity and latency requirements and therefore a potential solution might be that of $\mathrm{THz}$ wireless transmission as a wireless extension of the optical fiber between rural and highly populated urban areas to ensure high-speed connectivity everywhere in a cost effective manner [179]. Fronthaul links can be used to give broadband/high speed access to the end user when operating at higher frequencies at places where the broadband customer does not have fiber connectivity and where the link distance is much smaller compared to the backhaul. $\mathrm{THz}$ transmission can be applied to $5 \mathrm{G}$ fronthaul links in order to optimise and speed up the implementation of $5 \mathrm{G}$ connectivity in urban areas and thus; can provide more flexibility to avoid the expensive fibre connections currently required for $5 \mathrm{G}$ system deployments. Automatic planning algorithms [32], [180] for the backhaul/fronthaul links are essential in designing guidelines for sucessfull deployment of these links. Planning for these scenarios should involve identifying the shortest LoS link where highly directive antennas that will minimize multipath propagation will be employed. For terrestrial links we can assume that communications will be below the height of clouds; however, the effect of attenuation due to fog may be significant. The applicability and feasibility of such transmission has already been proven by MMIC based technology and demonstrated at large distances of $850 \mathrm{~m}$ at carrier frequencies of $240 \mathrm{GHz}$ where data rates of $64 \mathrm{Gbps}$ were achieved [181].

5) WLAN: Typical indoor communications in the $\mathrm{THz}$ band will involve scenarios such as office rooms and conference rooms. Such scenarios would have to consider LoS, link blockage, and scattering as the main propagation phenomena and will be mainly applied for interconnecting access points and not for user access. Notably, considering that the WiGig $60 \mathrm{GHz}$ standard [182] is of such limited range, then $\mathrm{THz}$ cannot be expected to go any further with communication links ranging up to 20 metres. Calibrated RT simulations were presented in [184] for indoor radio channels by taking account different frequency points within the evaluated range due to varying propagation conditions and a novel calibrating method was employed that uses both spatial and temporal information that significantly improve the accuracy of the RT tool [185] and extend its applicability to much wider frequency ranges. The RT tool developed was built upon common image theory for reflections [186] and Kirchhoff scattering [187] analysis and the results were further validated against measurement data collected at $300 \mathrm{GHz}$. Choi et al. in [188], proposed a novel GBSM for $\mathrm{THz}$ channels that mathematically describes the specular component of a single induced cluster from indoor reflections that can be utilized when the main LoS component is blocked. The model also accounts for the scattering loss and has the advantage of reduced complexity in comparison to RT. The model was verified against a mirror-image procedure to aid in evaluating the physical characteristics and evaluate the system-level performance of the THz channels. In [189], RT simulations were carried out in an indoor office room where clustering due to multipath reflections from materials' of known permittivity were identified and the effects of multipath propagation on the PAP and PDP were considered. The effects of clustering were validated against the SV model as they were found to follow an exponential decay pattern.

In [190] RT modeling of short-range wireless communications in a typical office environment was presented for the atmospheric windows below $1 \mathrm{THz}$. Statistical modeling was considered in terms of AoA, AoD and ToA and Up to $4^{\text {th }}$ order reflections were considered in the simulation. It can be deduced that LoS components have the greatest contribution in received power while in the NLoS case the power received from reflections was much less, though it could supplement the LoS when it is not available (e.g., due to blockage) but a high reliability communications system cannot be dependent on these components.

The effects of antenna misalignment on the received power will play a very important role at $\mathrm{THz}$ due to their high directivity, and as such, they are modeled as a random process in [191] where monte carlo simulations were used to evaluate these effects while RT was used to determine the resolvable paths and their associated gains and losses as a result of propagation in an indoor office room. Similarly, the effects of different antenna directivities on resolvable paths for different applications in terms of propagation losses, fading and delay spreads were investigated in [192] and it was found that antenna directivities for fixed P2P applications should be higher than applications with positional uncertainty such as communications on board a desktop in the presence of nonstationary devices such as laptops where lower directivities could cater for antenna misalignment.

In [193] indoor channel measurements were conducted in the recently standardised bands between $275-321 \mathrm{GHz}$ for $\mathrm{THz}$ communications in a typical office scenario. Calibrated RT was performed, and a spatial and temporal analysis of the results was provided based on measurement data. MIMO channel capacity was calculated for a $2 \times 2$ system where a capacity of $6.21 \mathrm{bit} / \mathrm{s} / \mathrm{Hz}$ was obtained. RT results were further compared against measurement data and were found to be in good agreement. Nonetheless; diffuse scattering was not considered in the analysis. A modified version of the SV model used to characterize the $\mathrm{THz}$ channel for indoor $\mathrm{THz}$ communications with antenna sub-arrays operating at $300 \mathrm{GHz}$ was presented in [194]. An upper bound on the ergodic channel capacity was obtained in relation to the number of antenna sub-arrays and as a function of distance where the capacity decreased with an increase in the distance and the array gain decreased with the increase in sub-array number. The ergodic channel capacity at a distance of $10 \mathrm{~m}$ with 40 antenna sub-arrays was found to be 0.125 Tbps. Stochastic modeling of the indoor propagation channel by considering both time and frequency domains [195] in a statistical analysis of ultra-wideband channels in the 275$325 \mathrm{GHz}$ band was provided for a realistic office scenario and the results were verified against calibrated RT simulations. The model accounts for AoA/AoD, ToA and phase together and considers channel dispersion for each resolvable path and so can be used to simulate practical MIMO systems. Angular spreads of a few degrees were observed while $3 \times 3 \mathrm{MIMO}$ capacities ranging between $5.5-7.3 \mathrm{bit} / \mathrm{s} / \mathrm{Hz}$ were measured.

Furthermore, Priebe et al. [196] conducted channel measurements at $300 \mathrm{GHz}$ with a $10 \mathrm{GHz}$ bandwidth. Mea- 
surements of diffraction and absorption coefficients of different materials placed between the transmitter and receiver were conducted such as glass, MDF and Plexi-glass where absorption coefficients of $9.98 \mathrm{~cm}^{-1}, 4.31 \mathrm{~cm}^{-1}$ and 2.59 $\mathrm{cm}^{-1}$ were measured, respectively. Measurements were also conducted for two separate indoor scenarios, a P2P link of devices on a desktop and the connection of a laptop to an access point within the office room. LoS and DirectedNLoS (DNLoS) propagation scenarios were considered up to $2^{\text {nd }}$ order reflections. An interference investigation was also demonstrated by using a two-ray model [197] while an empirical narrowband path loss model was proposed for the investigated scenarios. Important to mention, that DNLoS propagation that was initially proposed in [198], differs from the traditional concept of NLoS that is mainly used for sub-6 $\mathrm{GHz}$ communications as non-directed NLoS contributions to the received signal strength may be in many cases insignificant at THz. However, as we have mentioned previously for cases such as intra-device communications where wave propagation is confined to a small space, the NLoS components will play a major role. In [199] a coverage and achievable data rate analysis was conducted for an indoor environment at $\mathrm{THz}$ frequencies. A Single Frequency Network (SFN) was adopted where multiple AP's transmit the same information over the channel within the same frequency range. This can be used to combat the distance problem and extend the transmission range. The minimum data rates for each user are considered for different resource allocation schemes. The results show an increase in the minimum achievable data rates from $25 \%$ using a single AP to $95 \%$ using a total of 20 AP's.

Zhao et al. [155] extended THz indoor channel characterization to higher frequencies by providing results for the $650 \mathrm{GHz}$ band in comparison to the $350 \mathrm{GHz}$ band. An indoor scenario was considered with a $10 \mathrm{GHz}$ bandwidth at both the low band and high band. A temporal and spatial analysis was performed and from the results it was found that the NLoS component is sufficiently strong to compensate for the received signal in case of a moving user blocking the main LoS component, though achievable data rates were reduced by about $50 \%$ at both bands for the NLoS case in comparison to the LoS case. Channel dispersion effects were also considered with delay spreads of less than 80 ps being recorded due to the high directivity while hardly any diffraction was observed. It can be deduced that RT simulations may prove to be efficient at evaluating indoor channels at frequencies beyond $300 \mathrm{GHz}$ due to the high correlation between the identified paths and indoor environment, though knowledge of the materials present in the environment is required to obtain accurate results.

Shafie et al. [200] presented a 3D model accounting for the effects of dynamic user blockage, antenna directivity and interference caused by nearby access points by considering factors such as molecular absorption, the height of the devices and transmission distance. The effect they have on coverage was considered and the authors provide suggestions for performance enhancement considerations. A 3D dynamic channel model for tracking the LoS beam between user equipment and access point in an indoor scenario described by a Kalman filtering algorithm and Markov Process by considering varia- tions over time in the propagation channel was described by Nie and Akyildiz [201]. The results were further validated against RT simulations where the effects on the capacity were addressed generating satisfying results. A 3D MIMO nonstationary indoor GBSM was proposed by Wang et al. [202] where the channel parameters were varied spatially and over time and the impulse responses were generated. LoS, NLoS and diffuse scattering were considered in the model and different characteristics were studied in the form of time, space and frequency correlation functions. The theoretical GBSM developed was verified against simulations and were found to be in good accordance. A statistical model based on empirical data collected from measurements using a channel sounder recording over 15000 thousand PDPs and a detailed clustering analysis was presented by $\mathrm{Ju}$ et al. [203] that indicates cluster paths, powers and delays while the data collected was also validated using simulation for the cases of LoS and NLoS propagation.

When highly directive antennas are employed, even the slightest change of degree in movement may cause beam misalignment. In cases where the user e.g., is playing a game on his phone which requires dynamic rotational movements and micro-displacements, the effects of beam misalignment may severely affect the reliability of a high data rate link. Petrov et al. [204] characterized these micro interactions between the user and his mobile phone in terms of beam misalignment and its effect on the achievable data rates were modeled mathematically in the form of angular and Cartesian micro-mobility where it was found that these effects may have a serious impact on the capacity of the THz link while dynamic beam alignment in contrast to periodic alignment may increase the spectral efficiency by $10-15 \%$.

Peng et al. [205] introduced a novel 3D AoA estimation method for dynamic indoor $\mathrm{THz}$ channels using a forwardbackward algorithm for calculating the Bayesian interference. AoA movement in the algorithm was represented as a Markov process and the Bayesian interference was used to combine the likelihood and a priori information providing a better estimation rather than the likelihood itself. The model considers human movement in terms of translation and rotation of user equipment (i.e.- smartphone) from which the statistical probabilities were obtained. A ray launching simulator for Bayesian interference is adopted to generate the channel model for the indoor scenario. Going one step further, Peng et al. [206] proposed an AoA estimation model for dynamic indoor propagation that considers spatial correlations to describe the dynamic properties of a user moving within the given environment due to time information correlations resulting from the low user speed and statistical movement pattern as well as space information correlations due to similar user displacement in case of a MIMO scenario. The Bayesian filter was fitted to improve accuracy of estimation of AoA, while simulation results show that this cooperative estimation method considering space and time correlations can improve the performance in each scenario. Very Recently, A two-step AoA estimation method was also devised by Peng et al. [207], to solve the problem of received power due to unknown locations of user equipment and access points. After initial 
AoA is estimated by considering that the power received over different angles is correlated over different frequencies. In this way the model could be applied at lower frequencies, and then used to provide a rough estimation at the $\mathrm{THz}$ range, thus, reducing computational complexity which is much higher at THz. The results were also validated using channel sounding measurement data.

The unavoidable densification of access points expected at $\mathrm{THz}$ because of the limited output power and need for high gain and antennas that limit the communications distance requires efficient deployment to avoid complexity and resource limitations. Singh and Sicker [208] addressed these effects by proposing efficient access point deployment through numerical modeling considering the maximum radius of coverage to minimize the number of access points deployed and the use of repeaters to assist for coverage. Similarly, Shafie et al. [209] considered access point densification and its effects on maximum achievable capacity. A 3D model was developed that considers basic propagation characteristics along with access point locations and human movements that block the signal modeled as a Poisson processes. The model framework consists of LoS as well as reactive connectivity modeling where users switch between access points in case of blockage, hence, overcoming molecular absorption effects and enhancing link reliability.

6) HST Communications: In a new era of smart, green and intelligent transport, high data rate communications and high speed broadband access to HST users is expected to be delivered by the next generation of mobile communications to realize the vision of "smart rail mobility" that consists of communications between trains, passengers and infrastructure [33]. The most promising application of $\mathrm{THz}$ however, is in the Train-to-Infrastructure (T2I) channel to deliver very high data rates of $100 \mathrm{Gbps}$ etc. but with very narrow beamwidths in comparison to mmWave. Regarding the intra-wagon channel, for links between users and access points, the large bandwidth at $\mathrm{THz}$ could be potentially exploited in the future for high data rate transmissions and to accommodate a large number of users though mmWave should be sufficient to provide HD video applications at present [33].

For any given complex communications scenario that includes a high order of mobility, new paradigms of channel modeling have to be developed. Due to limited channel sounding, there is a need for extensive calibrated ray-tracing simulations in order to develop the stochastic channel model. Limited measurements means a limited degree of freedom as full dimensional channel information are required from measurement directly and hence, the need for simulation. For instance, The T2I inside-station channel was characterized in [210] for the first time. The measurements were conducted using a channel sounder at $304.2 \mathrm{GHz}$ centre frequency with $8 \mathrm{GHz}$ bandwidth. The results extracted from the PDP were presented in terms of Rician k-factor and RMS delay spread. Two scenarios were considered to emulate the channel when the train is stopping and a ray-tracing simulator was developed for the given scenarios that was calibrated bases on the measurements. The results obtained show that the $2_{\text {nd }}$ order of reflection is sufficient to account for in the absence of the main LoS component while the inner wall of wagon when the door opens can reflect multipaths with unexpected long delays and has to be considered before system deployment.

Guan et al. in [211], employed calibrated-RT simulations to investigate the intra-wagon scenario for the first time. Similar to the inside-station channel the $2_{\text {nd }}$ order reflections were found to be strong enough to compensate the main delay component. In order to make something useful out of this characteristic of HST channels, some reflectors or a Reconfigurable Intelligent Surface (RIS) [212] can be used in a DNLoS scenario to generate the first order reflection when the LoS path is not available. For system design there may be some special propagation elements to consider such as multipaths arriving with the same delay but from very different directions. Onward, an detailed analysis of the calibration and validation processes for the RT simulator was presented in [213] and extended to a wider range of scenarios where the Rx positions were varied and for a greater degree of freedom. From the analysis it is obvious that at $\mathrm{THz}$, propagation characteristics differ from the lower bands and channel multipath sparsity is a major characteristic with much smaller delay spreads expected at $300 \mathrm{GHz}$ when compared to the $60 \mathrm{GHz}$ band. In addition, Furthermore, Guan et al. [214] presented a capacity analysis for all five smart rail mobility applications identified at 300 $\mathrm{GHz}$.

7) Inter-Satellite Links: The increasing demand for improved coverage in the Internet-of-Things (IoT) field has encouraged the industry to support research to achieve a highly reliable and fast communications network to deliver space and aerial links on top of the existing terrestrial networks. This is expected to be achieved by the deployment of CubeSats/nanosatellites that are preferred to conventional satellites typically deployed in Low-Earth-Orbit (LEO) Medium-EarthOrbit (MEO) and Geosynchronous-Equatorial-Orbit (GEO) constellations [215] as they are lower in size, weight and cost. Currently, communications within the LEO, MEO and GEO constellations are accommodated within the microwave bands up to $75 \mathrm{GHz}$ while they are also achieved within the optical bands using lasers. Due to the saturated spectrum in the microwave bands [216] where these constellations operate as well as the limitations in beam alignment to achieve LoS propagation and synchronisation of satellites within the optical bands [217], an alternative solution is required. The $\mathrm{THz}$ band poses as a candidate for future ISL links as it holds a number of advantages. Firstly, atmospheric attenuation in the uppermost layers of the earth's atmosphere is a lot less prominent due to the thin air, while effects such as rain, cloud and fog are non-existent resulting in much longer communications distances. Furthermore, the $\mathrm{THz}$ band can provide higher data rates and capacity than the lower bands while it holds less strict beam alignment requirements in comparison to communication links operating within the optical bands. Nevertheless, channel propagation for ISL's differ in comparison to terrestrial links. Channels that have to be considered for ISL deployment include the near-earth channel and the deep-space channel. Parameters that are used to describe propagation within the ionosphere in the near-earth channel include the plasma frequency, collision frequency and depolarization of 
electrons and ions caused by the Faraday rotation [218]. Furthermore, for deep-space channel propagation, factors that require consideration involve cosmic microwave background radiation, noise effects due to solar brightness, and solar effects such as flares and wind.

Some early studies have explored the possibilities of ISL communications [219], [220], and a capacity analysis in [221] for an ISL was conducted where $100 \mathrm{Gbps}$ were achieved at a relatively short-range communications distance of $1 \mathrm{~km}$ operating at $340 \mathrm{GHz}$ and $2.5 \mathrm{Gbps}$ at $100 \mathrm{~km}$ inter-satellite distance, with the later requiring a $62 \mathrm{dBi}$ gain antenna, $50 \mathrm{~cm}$ aperture area and an output power of $1 \mathrm{~W}$. Nie et al. [222], considered the propagation effects present in near-earth and deep-space channels and developed a channel model to investigate diversity techniques used to achieve higher throughput. Their analysis has shown that near-earth channel propagation in the ionosphere does not affect the $\mathrm{THz}$ link significantly while for the deep-space channel only major solar activity can cause angular instabilities. Large Doppler variations due to motion of the satellites are also expected as observed using FDTD simulations in [223]. In [224] a performance analysis for LEO ISL's was conducted where it was found that the Rician fading channel model best describes the channel in terms of LoS propagation and Doppler variations while results were presented in terms of packet arrival rate and violation probability indicating the impact they have on backlog and delay performance. Finally, diversity techniques such as space and polarization diversity as explored in [225] should be carefully utilized to maximize the throughput.

8) V2X Communications: The next generation of mobile communications is one where it is expected that the requirements of $\mathrm{V} 2 \mathrm{X}$ communications will be fulfilled. A network that is extremely intelligent and able to conquer the requirements of high data rates, ultra-fast, ultra-reliable and ultra-low latency exchange of information is a future vision for 6G V2X communications [226]. The need for enhanced road safety and inter-connectivity that satisfies the requirements mentioned, directs us to communications in the $\mathrm{THz}$ band that are vital in achieving huge amount of throughput, though ultrareliable information exchange may be difficult to achieve with the current technology [227]. Particularly, highly directive Thz links in the lower end of the spectrum may be utilized for large information exchange within vehicular fleets of semiautonomous vehicles performing cooperative driving [228] as well as for data relaying in congested networks to enhance connection reliability [229]. Efforts in developing models at $\mathrm{THz}$ for $\mathrm{V} 2 \mathrm{X}$ communications so far have been limited by advancements in technologies of transceiver design, new waveforms, design of phased arrays along with dynamic beamforming and beam-steering. Therefore, taking into consideration such Doppler and beam-forming capabilities being still too bad at mmWave, at $\mathrm{THz}$ it may be necessary to have such communications between vehicles at points where they are stationary, such as to do 'vehicle kiosk downloading/uploading'.

Petrov et al. [230], conducted an interference study that considered the impact in terms of direct transmission and multipath reflections from vehicles on the side lane for an urban and highway V2V communications scenario at 300
GHz. A 2D geometric model was developed to predict the interference and it was found that the interference largely depends on the angle of the antennas radiation pattern where for angles greater than $40^{\circ}$ multipath reflections from the side lanes contribute significantly to the interference, while for angles less than $20^{\circ}$ the interference caused was minimal. Then, Eckhardt et al. [231] conducted a detailed propagation analysis based on measurement data obtained at $300 \mathrm{GHz}$ for $\mathrm{V} 2 \mathrm{~V}$ communications in a stationary environment. The scenarios considered include LoS propagation in a singlelane scenario and reflections from nearby vehicles for various multi-lane scenarios as well as under-vehicle propagation. These measurements may contribute to future propagation modeling for vehicular communications. Nonetheless, more complex scenarios have to be investigated in the future that take into consideration factors such as road surface properties, vegetation and a large number of mobile vehicles. In [232], an initial study of signal losses resulting from material penetration and reflections at $300 \mathrm{GHz}$ for various vehicular scenarios was conducted, though a much more detailed analysis is required and for a greater range of scenarios.

Busari et al. [233], described a generalized 3D model for communication between mobile users cars and infrastructure for an outdoor scenario by making use of the hybrid beam forming architecture to realize massive MIMO. The model considers a single-path LoS channel where different equidistant access points are placed along the road with a fixed speed for the mobile user and vehicle. Large and small scale fading were investigated along with the Doppler frequency. Moreover, Yi et al. [234] examined Vehicle-to-Infrastructure (V2I) channel for urban and highway scenarios at $300 \mathrm{GHz}$ with $8 \mathrm{GHz}$ bandwidth. Measurements were conducted using a SC channel sounder and RT was employed for the analysis of the propagation environment. The attenuation due to rain and snow in the propagation channel was also considered as it contributes to the path loss and were found to be $26 \mathrm{~dB} / \mathrm{km}$ and $348 \mathrm{~dB} / \mathrm{km}$, respectively. Notably, the attenuation due to snow completely differs from what is expected from the theory presented in section II where it is expected to be similar to that of rain, indicating that more measurements need to be taken to account for this effect. The authors have concluded that future works need to account for traffic flow since LOS communication cannot be guaranteed between different vehicles. A time-varying propagation model that aims at characterizing the V2I urban channel was proposed in [235]. The timevarying characteristics were captured using a Birth-Death (BD) process in terms of the multipath behavior of the channel within an RT model. Based on the RT model, a time-varying GBSM was developed that considers multipath propagation as a result of specular reflections and spherical wave propagation from which a statistical analysis was derived.

A 3D channel model based on propagation resulting from a reflect-array antenna composed by graphene material that is dynamically reconfigurable by tuning its chemical composition was presented in [236] that considers LoS, NLoS and scattered paths which could be adopted in vehicular networks. The model which was simulated using RT techniques, due to the presence of the reflect-array achieved a delay spread reduction 
by over $1 \mathrm{~ns}$ to less than $0.1 \mathrm{~ns}$ showing an improvement to the coherence bandwidth and very small angular spreads $<0.12^{\circ}$ that were one tenth of the spreads found without beam-forming. However, the beam-forming gain of $18 \mathrm{~dB}$ achieved in the main direction requires a very small variation in beam alignment angle of $<11^{\circ}$.

9) D2D Communications: D2D communications can be considered as a promising technology by which the spectral efficiency within a cellular network can be maximised by utilizing devices that are in close proximity to each other without the need to go through the Access Points (AP's) or base stations (BS's) [237]. The communicating devices can share resources efficiently in order to achieve higher data rates, reduced latency and higher throughput in order to improve the Quality-of-Service (QoS) and enhance user experience [238]. It has several use cases including local services, emergency communications, mobile tracking and positioning, extending cellular coverage etc. An envisioned scenario would be devices communicating at a very short-range (e.g. two devices placed on the same table) in a Wireless Personal Area Network (WPAN). Toward realizing D2D communications in the $\mathrm{THz}$ band, there are certain challenges faced in characterizing D2D propagation channels that have to be addressed. Some of these challenges include neighboring device discovery, mobile device tracking, managing interference between neighboring devices and enhancing the link reliability [36].

Abbasi et al. [239], presented urban double-directional measurements for D2D applications in the 140-141 GHz band covering distances up to $15 \mathrm{~m}$ on a linear route. A statistical analysis was carried out in the spatial and angular domains where delay spreads and angular spreads were calculated. RMS delay spread was found to have a relation with direction with small delay spreads in low power directions and larger delay spreads in high power directions recorded, while angular spreads were found to increase by $5 \times$ at the end of the route compared to the beginning.

Kim and Zajić described a 2D GBSM for short-range D2D communications in [240]. The geometric model consists of concentric sectors with the presence of uniformly distributed scatterers around the Tx and Rx and accounts for LoS, and NLoS while considering first and second order rays. Based on the geometric model, a time-invariant statistical model was developed for short range multipath fading channels that was analysed in terms of PDP and frequency correlation function. To validate the model two scenarios were considered; the first scenario considered LoS desktop propagation at a centre frequency of $300 \mathrm{GHz}$ with $20 \mathrm{GHz}$ bandwidth and the second scenario involved NLoS desktop propagation in the D-band (110-170 GHz). The results were compared with theory and simulated using a novel sum-of-sinusoidal method were a good agreement has been found with the approximations of the geometric model.

10) Nano-Networks: The $\mathrm{THz}$ channel has been found to be a candidate for many applications including those in the medical domain. For modeling the channel for in-vivo applications, the human tissue needs to be considered. Path loss can be computed based on different molecular characteristics, scattering from cells within the tissue, distance between the nano-machines and frequency of operation. Different models have recently been developed for in-vivo nano-machine communication within the human tissue. Jornet and Akyildiz [241] presented a channel modeling and capacity evaluation for EM wireless nano networks in the $\mathrm{THz}$ band between 0.1-10 THz. They assumed that since communication between nanomachines will be made over a very short range, the concepts of molecular absorption should be heavily accounted for and the concept radiative transfer theory [242] was employed for modeling the path loss and system noise while the model developed can be easily modified fur use in various nanonetwork applications as it takes contributions from different concentrations and types of molecules. In [243] a channel model along with a link budget analysis was presented for In vivo Wireless Nano Sensor Networks (iWNSN's) in the $\mathrm{THz}$ band. Propagation phenomena inside the human body were analysed mathematically, the path loss was computed while taking into account molecular absorption from human tissue, wave scattering from body particles and the effects of spreading on the propagation of the wave. The results of the model were validated through electromagnetic wave simulations. The model developed may facilitate the design and deployment of future iWNSNs.

Yang et al. [244] investigated the characteristics of wave propagation inside the human body in the $\mathrm{THz}$ band and performed an initial study of the nano-network system performance. A path loss model was developed, and the results were verified through software simulations. Based on the path loss and noise level for propagation of the wave, the channel capacity was studied to give an insight on the performance of nano machines within the nano-network showing that at very small distances $(\sim \mathrm{mm})$ rates as high as 100 Tbps can be achieved. Abbasi et al. [245] proposed a statistical path loss model for channel characterization inside the human tissue for In-Vivo networks. The model aims to provide a deeper understanding of wave propagation inside the human tissue and the losses associated with it. It considers the frequency of operation, the distance separating nano-machines within the network and number of sweat ducks in the form of a numerical skin model considering the different skin layers and their thickness. The accuracy of the channel model proposed was validated by comparing the results to simulation and THz-TDS measurements of the skin sample between 0.1-4 THz. The statistics of error prediction in terms of standard deviation and mean were presented and the results were found to agree well with the measured ones. A stochastic model that considers different forms of noise that may impact propagation within nano scale systems was considered by Elayan et al. [246] by deriving probability distributions of the noise sources. It was found that Doppler induced noise has the greatest contribution compared to other sources of noise such as black body noise and Johnson-Nyquist noise. Vegni and Loscri [247] investigated the chiral parameters such as DNA chains and biomolecules and their effects on LoS and NLoS propagation as well as their effects on the capacity within a given medium in the $4-10 \mathrm{THz}$ frequency range where the frequency selectivity at $\mathrm{THz}$ creates an effect known as Giant Optical Activity (GOA) [248]. 
11) General Models: In this part we present some noteworthy modeling contributions that target to extract the main channel characteristics at $\mathrm{THz}$ and which can adapt to a wide range of scenarios. Han et al. [249] presented a multi-ray deterministic model that was developed based on RT techniques for the frequency range of 0.06-10 THz. A narrow-band model was initially created and the multi-ray model was formed based on superposition of the sub-bands. A thorough analysis on propagation effects such as LoS, scattering, diffraction, reflection and broadening effects along with common theory such as Kirchhoff scattering and knife edge diffraction were employed to analyze simulated results by varying the distance and observing the frequency selectivity of the channel. RMS Delay spreads of $0.19 \mathrm{~ns}$ were found at $0.3 \mathrm{THz}$ that dropped significantly to $51.7 \mathrm{ps}$ at $0.7 \mathrm{THz}$ over a $3 \mathrm{~m}$ distance resulting to coherence bandwidths of $1.06 \mathrm{GHz}$ and $3.87 \mathrm{GHz}$ respectively. The broadening effects were found to increase with an increase in frequency because of the higher frequency selectivity resulting from the short wavelength, hence, limiting the achievable data rates. The wideband channel capacity was also estimated over the $0.06-1 \mathrm{THz}$ range using the water-filling power allocation method to be $75 \mathrm{Gbps}$ with a $10 \mathrm{dBm}$ power at a $3 \mathrm{~m}$ distance. The simulated results were compared against experimental measurements found in literature below $1 \mathrm{THz}$ where some material properties are accessible, though at higher frequencies the frequency selectivity is highly unpredictable to assume the validity of the model. Moldovan et al. [250] proposed an indoor channel model for 0.1-10 THz considering LoS and NLoS propagation where specular as well as diffuse roughness components were considered. The LoS component was found to be dominant even though at larger distances the NLoS component becomes more significant and in some cases dominant due to constructive interference between the indirect paths. Data rates of $1 \mathrm{Tbps}$ were simulated over a $1 \mathrm{~m}$ distance with a power of $1 \mathrm{~W}$, while the capacity of just the NLoS component was found to be $100 \mathrm{Gbps}$ when modeled by a water-filling approach between 0.1-1 THz. Tsujimura et al. [251] illustrated a casual channel model for the THz band. This work aimed to address the issue of deriving the impulse response from transmittance by assuming linear phase that leads to a symmetric impulse response before and after the most significant LoS component where it is not realistically possible for the signal to arrive before the main delay tap since it makes the model noncasual. The model developed can address this issue and was validated with THz-TDS measurement data $<2 \mathrm{THz}$ that agree well with the results. A variation can be observed in the coherence bandwidth obtained from the impulse response as a function of the centre frequency that may be used to determine the frequency selection for wireless communication systems. A generalised 3D THz channel model was presented in [252] for 6G Ultra-Massive MIMO wireless communication systems that considered non-stationarities in the channel and and cluster evolution in frequency, space and time domains and which can be applied for a variety of applications such as indoor, D2D communications.

12) Surface Roughness Modeling: Surface roughness is a measure of the finely spaced micro-irregularities on the surface texture which is composed of three components namely, roughness, waviness, and form [253]. The effect surface roughness will have on the scattering behavior depends on the surface height relative to the wavelength. Factors that describe the roughness of a surface are the RMS height that allows for a fair comparison of the roughness of materials with similar structures and correlation length which is the length from a point on the surface to a short distance where the heights of the rough surface are correlated with each other.

In [254] rough surface scattering measurements were conducted in the low $\mathrm{THz}$ range for automotive radar applications at frequencies up to $1.1 \mathrm{THz}$. Back-scattering coefficients were measured and varied over the angle of incidence, to determine the reflective properties of sandpaper of known permittivity at different grit levels. The backscattering coefficients were measured at frequency of $300 \mathrm{GHz}$ over a bandwidth of 8 GHz. The authors of the paper have deduced that by increasing the frequency, the EM roughness of an individual surface also increases causing diffuse scattering and an increase in the signal back-scattered from the road surface. In addition, the backscattering coefficient decreases significantly as the incidence angle increases. Furthermore, in [255] the authors examined the backscattering of EM signals at frequencies of $300 \mathrm{GHz}$ and $680 \mathrm{GHz}$ with $4 \mathrm{GHz}$ bandwidth from surfaces with different roughness. The backscattering coefficients were measured for over a range of incident angles for different samples of sandpaper of grit levels 50, 120 and 220 . At $300 \mathrm{GHz}$ the backscattering from sandpaper with grits 120 and 220 were found to be specular and the signal quickly decreased with the increase in angle. At $680 \mathrm{GHz}$, backscattering from sandpapers with grit 50 and grit 120 were diffuse, while backscattering from sandpaper with grit 220 combines specular and diffuse reflections.

In [256] the roughness parameters of metallic surfaces were characterized using $\mathrm{THz}$ reflection spectra. Kirchhoff's approximation was used as a theoretical model and was compared to the results obtained from measuring the reflection spectra of aluminium using Fourier transform spectroscopy. The RMS roughness levels were measured, and a good agreement was found between the theoretical and experimental results. The reflectance measurements were made using a spectrometer while the RMS roughness was measured using a stylus surface profilometer. The range of frequencies considered in the experiment varied between 0.3 to $3 \mathrm{THz}$. The results were presented in terms of reflectance and wavenumber where the RMS roughness curve was compared to the Gaussian fit.

In [257] a theoretical and empirical evaluation of the effects sub-micrometer roughness has on the effective conductivity in the $\mathrm{THz}$ regime was presented. Two frequency ranges were considered at 400 and $650 \mathrm{GHz}$ that were achieved using an open quasi-optical resonator where the $\mathrm{THz}$ waves are usually incident to the sample. The statistical analysis performed using the measurement data was validated using two closed-form models and a finite-element simulation. It was found that the Mie-scattering based and finite element approaches are more accurate for smoother samples relative to the skin depth. For surface considerations greater than the skin depth it was 
found that the Hammerstad and Bekkadal models provide more accurate predictions of effective conductivity. The results presented in this paper can be set as benchmark for new and improved models of effective conductivity in the $\mathrm{THz}$ regime.

In [258] the influence of surface roughness on $\mathrm{THz}$ reflection measurements is considered. This paper aimed at providing an insight in the performance of THz TDS for the detection of hazardous substances for security applications. The measurements undertaken involve smooth and rough metallic and dielectric surfaces varied over angle of incidence and degree of surface roughness (grit). A Maitai femto-second laser in a THz-TDS setup was employed for the measurements. The frequency range considered was $0.1-3.0 \mathrm{THz}$ and the results were validated based on radar theory.

In [259] a study was performed to provide an insight to the different measurements conducted used to determine the properties of different material in the $\mathrm{THz}$ channel. The absorption coefficient and the refractive index were calculated for the frequency range of 70 to $110 \mathrm{GHz}$. Surface roughness measurements were also performed for frequencies beyond $100 \mathrm{GHz}$ (up to $410 \mathrm{GHz}$ ) to predict reflection and scattering of the wave more accurately by using equipment available for optical 3D micro and nano-metrology [260].

In [261] rough surface scattering was investigated for both indoor and outdoor scenarios at carrier frequencies of 100, 200, 300 and $400 \mathrm{GHz}$. NLOS and LOS scenarios are considered based on signal reflections from building walls. The Bit Error Rate (BER) performance was measured relative to the output power. It was shown that the losses caused by scattering off the rough surface of the walls were much less than the losses caused by absorption at all measured carrier frequencies, while the losses caused by absorption were seen to increase with frequency. To achieve error-free transmission the transmitted power needs to be higher to account for absorption and scattering.

\section{A. Summary for 6G Channel Modeling Efforts}

Table III summarizes and compares the most important scenario-specific modeling contributions found in literature. Since channel models in the $\mathrm{THz}$ band mainly rely on channel measurements, many more measurement campaigns must be undertaken in order to realize $6 \mathrm{G}$ applications and make use of the vastly available spectrum between $0.1-3.0 \mathrm{THz}$ for future communication systems design. Having a closer look, we can deduce that these efforts lack characterization of the channel particularly above $300 \mathrm{GHz}$ and are quite sparse for some of the envisioned applications. Furthermore, it is obvious that there is a lack of sounding equipment with MIMO capabilities that are imperative for realizing long distance communications and achieving high throughput levels. A more thorough analysis regarding requirements of future channel modeling efforts follows in section VII.

\section{Challenges and Future Research Directions}

\section{A. Open Issues and Challenges}

From the work analyzed in this survey paper, there are some open issues and challenges in modeling techniques that need to be improved upon while other future research directions are set.

1) Diffuse Particle Scattering: Models such as R-R and $\mathrm{B}-\mathrm{K}$ that are used to characterize scattering from material surfaces may no longer be applicable and therefore require further consideration at THz. The R-R model while it performs well at predicting scattering from various angles at generally smooth surfaces and takes into consideration polarization of the waves, it fails doing the same for rough surfaces. On the other hand, the B-K model performs better for rough surfaces but is not as effective at wider angles due to effects such as absorption and shadowing, while it does not account for effects such as polarization [262]. Also very important at $\mathrm{THz}$ is that there is a very thin Fresnel zone. When different echoes arrive but the Fresnel zone is blocked by scatterers, this causes quite separate specular rather than diffuse reflections of some rough surfaces as diffuse scattering happens where the tip of the Fresnel zone is incident on the rough surface. This is an important characteristic that opens up more work.

2) Atmospheric Channel Dispersion: Atmospheric Group Velocity Dispersion (GVD) is a broadening effect observed in $\mathrm{THz}$ atmospheric channels which can affect significantly the maximum achievable data transmission rates [263]. GVD is a measure of how different channels, media may affect the duration of the pulse propagating through them. As the $\mathrm{THz}$ channel is dominated by atmospheric effects such as water vapor, the resonances occurring within the water particles introduce GVD due to different frequency components propagating at different velocities. Another property that requires consideration are the Doppler shifts that may also cause the broadening of $\mathrm{THz}$ pulses in presence of moving scatterers within a multipath environment and is extremely important when modeling radar sensing applications such as vehicle tracking [264]. Since the $\mathrm{THz}$ channel is highly frequency selective, GVD is caused leading to a distortion and broadening of the pulse waveform along with Inter-Symbol Interference (ISI). Factors that may limit transmission rates and cause GVD that need to be explored further include link distance, water vapor density and channel bandwidth. In [265] a novel method is developed for compensating atmospheric channel dispersion for $\mathrm{THz}$ wireless communication using a cohort of stratified media reflectors.

3) Capturing Time-Varying Channel Characteristics: So far channel characterization was mainly focused on static scenarios where P2P links will be employed. But in reality most cases will have to adapt to dynamic variations of the channel characteristics due to the highly directive characteristic of the $\mathrm{THz}$ waves. Link blockage will be a major issue, especially when planning for deployment of $\mathrm{THz}$ communications in scenarios where the presence of moving scatterers would affect coverage massively or in scenarios where dual mobility is involved when two devices are communicating with each other, i.e.- D2D communications. Moreover, in more advanced cases, i.e.- two vehicles communicating with each other, where both the $\mathrm{Tx}$ and $\mathrm{Rx}$ side are moving and at very high speeds, significant channel variations would occur continuously and large Doppler shifts would have to be accounted for over extremely short periods of time. With 
TABLE III

Important Modeling EFforts Characterizing the Terahertz Channel for 6G Wireless Communication ApPliCATIONS.

\begin{tabular}{|c|c|c|c|c|c|c|c|}
\hline Ref & Application & Scenario & Freq. (GHz) & Modeling approach & Antenna & Channel Statistics & $\begin{array}{l}\text { Measurement Cam- } \\
\text { paign }\end{array}$ \\
\hline $\begin{array}{c}\text { [163], [29], } \\
{[162]}\end{array}$ & Kiosk download & $\begin{array}{l}\text { LoS, parallel, tilted, } \\
\text { tilted and parallel, } \\
\text { multiple order reflection }\end{array}$ & $220-340$ & RT-stochastic & SISO & $\begin{array}{l}\text { Rician K-factor, RMS delay spread, } \\
\text { fading amplitude, ToA, AoA, AoD, } \\
\text { BER, path loss }\end{array}$ & VNA \\
\hline [164] & Desktop download & LoS, varying receiver angle & $\begin{array}{c}50-75,75-110, \\
110-170,140-220 \\
220-325\end{array}$ & Stochastic & SISO & ToA, AoA, fading amplitude & VNA \\
\hline$[119],[110]$ & Indoor & LoS, Specular reflection, scattering & $500-750$ & Stochastic & SISO & PDP, fading amplitude, ToA & VNA \\
\hline $\begin{array}{c}{[166],[167],} \\
{[30]}\end{array}$ & Data center & $\begin{array}{l}\text { LoS, NLoS, DNLoS, Rack-to-Rack, } \\
\text { Blade-to-Blade }\end{array}$ & $300-312$ & Stochastic & $\begin{array}{l}4 \times 4 \text { MIMO, } \\
\text { SISO }\end{array}$ & $\begin{array}{l}\text { path loss, shadowing gain, RMS } \\
\text { delay spread, doppler shift, } \\
\text { Rician K-factor, delay dispersion, } \\
\text { coherence bandwidth }\end{array}$ & VNA \\
\hline$[168]$ & Data center & $\begin{array}{l}\text { inter-rack, intra-rack, top of rack, } \\
\text { P2P }\end{array}$ & $300.2-308.2$ & Stochastic & SISO & $\begin{array}{c}\text { AoA, AoD, ToA, FSPL, } \\
\text { path attenuation, polarization }\end{array}$ & SC \\
\hline [169] & Data center & LoS, NLoS & $300-350$ & RT-stochastic & SISO & $\begin{array}{l}\text { Delay spread, angular spread, } \\
\text { amplitude fading, PL-angle correlation, } \\
\text { PL-delay correlation, phase and } \\
\text { frequency dispersion }\end{array}$ & VNA \\
\hline$[170]$ & Data center & LoS, scattering & $190-310$ & theoretical & SISO & $\begin{array}{l}\text { atmospheric absorption, path loss, } \\
\text { capacity }\end{array}$ & - \\
\hline$[156]$ & Data center & LoS, NLoS, OLoS & $300-320$ & Stochastic & SISO & $\begin{array}{l}\text { Delay spread, path loss, cluster powers, } \\
\text { shadowing gain, correlation }\end{array}$ & VNA \\
\hline [172], [31] & Chip-to-Chip & LoS, DNLoS, NLoS & $300-314$ & Stochastic & SISO & $\begin{array}{l}\text { Path loss, delay spread, coherence } \\
\text { bandwidth, amplitude fading }\end{array}$ & VNA \\
\hline$[176]$ & Intra-device & LoS, NLoS & $270-320$ & Stochastic & SISO & Amplitude fading, delay spread & VNA \\
\hline$[177]$ & WiNoC & & $0-1.0 \mathrm{THz}$ & Deterministic & & $\begin{array}{l}\text { path loss, capacity, BER, ISI, } \\
\text { field intensity }\end{array}$ & - \\
\hline$[178]$ & OWiNoC & NLoS & & FDTD-empirical & & Free space path gain, link budget & Laser source \\
\hline$[32]$ & Backhaul & LoS & $275-325$ & RT & SISO & $\begin{array}{l}\text { Interference, SINR, rain, fog and cloud, } \\
\text { atmospheric attenuation }\end{array}$ & - \\
\hline [181] & Urban & LoS & $224-256$ & NGBSM & SISO & $\begin{array}{c}\text { atmospheric attenuation, rain } \\
\text { and fog attenuation, path loss, error } \\
\text { vector magnitude }\end{array}$ & - \\
\hline$[116]$ & Urban & LoS, NLoS & $141-148.5$ & NGBSM & SISO & $\begin{array}{c}\text { Path loss, AoA, AoD, } \\
\text { angular spread, cluster spread }\end{array}$ & \\
\hline [184] & Indoor & LoS, NLoS, scattering & $300-310$ & Calibrated-RT & SISO & $\begin{array}{c}\text { AoA, AoD, ToA, delay spread, } \\
\text { cost function, vertical/horizontal } \\
\text { polarizations. }\end{array}$ & VNA \\
\hline$[188]$ & Indoor & LoS, NLoS, DNLoS, scattering & 300 & GBSM & SISO & $\begin{array}{l}\text { Atmospheric attenuation, path loss, AoA } \\
\text { in azimuth and elevation, clustering }\end{array}$ & - \\
\hline$[189]$ & Indoor & LoS, NLoS & 300 & RT & SISO & AoA, AoD, ToA, clustering & - \\
\hline [190] & Indoor & LoS, NLoS & $\begin{array}{l}300,350,410 \\
670,850\end{array}$ & NGBSM, RT & SISO & FSPL, atmospheric attenuation, ToA & - \\
\hline [191] & Indoor & LoS, NLoS & 300 & RT & SISO & path loss, SNR, BER, antenna gains & - \\
\hline [192] & Indoor & LoS, NLoS & 300 & RT & SISO & $\begin{array}{c}\text { amplitude fading, delay spread, } \\
\text { propagation losses, antenna directivities, } \\
\text { path loss, k-factor }\end{array}$ & - \\
\hline$[193]$ & Indoor & LoS, NLoS & $275-321$ & Calibrated-RT & $2 \times 2$ МाMО & $\begin{array}{l}\text { AoA, AoD, ToA, path loss, delay } \\
\text { spread, angular spread, polarization }\end{array}$ & $\mathrm{SC}$ \\
\hline [194] & Indoor & LoS & 300 & NGBSM & MIMO & $\begin{array}{l}\text { Capacity, array gain, clustering, ToA, } \\
\text { path gain, AoA/AoD, array stering } \\
\text { vector }\end{array}$ & - \\
\hline [195] & Indoor & LoS, NLoS & 300 & Calibrated-RT & $3 \times 3$ MIMO & $\begin{array}{l}\text { amplitude fading, delay spread, } \\
\text { angular spread, k-factor, vertical, } \\
\text { horizontal polarizations }\end{array}$ & VNA \\
\hline [196] & Indoor & LoS, NLoS & 300 & GBSM & SISO & $\begin{array}{l}\text { Interference, amplitude fading, } \\
\text { diffraction, ToA, AoA, AoD,path loss, } \\
\text { FSL, delay spread }\end{array}$ & VNA \\
\hline$[155]$ & Indoor & LoS, NLoS, scattering & 350,650 & Calibrated-RT & SISO & $\begin{array}{c}\text { Clustering, path loss, AoA, AoD, } \\
\text { SNR, Capacity, delay spread, coherence } \\
\text { bandwidth }\end{array}$ & VNA \\
\hline [200] & Indoor & LoS, NLoS & 1070 & GBSM & SISO & $\begin{array}{l}\text { Molecular absorption, interference, } \\
\text { coverage probability, SINR, polarization, } \\
\text { blockage, AP-UE distance, directivity }\end{array}$ & - \\
\hline$[195]$ & Indoor & LoS, NLoS & $60-10000$ & RT & SISO & $\begin{array}{l}\text { K-factor, capacity, path gain, path loss, } \\
\text { doppler }\end{array}$ & - \\
\hline [202] & Indoor & LoS, NLoS & $300,325,350$ & GBSM & M-MIMO & $\begin{array}{l}\text { Autocorrelation, spatial correlation, } \\
\text { frequency correlation, non-stationarity, } \\
\text { AoA, AoD, cluster power and delay, } \\
\text { fading amplitude }\end{array}$ & - \\
\hline [203] & Indoor & LoS, NLoS & 140 & NGBSM & MIMO & $\begin{array}{l}\text { Delay spread, angular spread, path loss, } \\
\text { number of clusters, cluster delays and } \\
\text { powers, ToA, AoA, AoD, spatial lobe } \\
\text { and subpath number and power }\end{array}$ & $\mathrm{SC}$ \\
\hline [204] & Indoor & LoS & - & GBSM & SISO & $\begin{array}{l}\text { Capacity and outage, micro-mobility } \\
\text { rotational effects and beam powers }\end{array}$ & - \\
\hline $\begin{array}{c}{[205],[206],} \\
{[207]}\end{array}$ & Indoor & $\begin{array}{l}\text { Sitting with smartphone, walking } \\
\text { with smartphone, notebook } \\
\text { on legs,LoS,NLoS }\end{array}$ & 300 & $\begin{array}{l}\text { Ray launching, } \\
\text { empirical }\end{array}$ & SISO,MIMO & $\begin{array}{l}\text { Bayesian interference, dynamic } \\
\text { AoA estimation, shadowing, } \\
\text { log-likelihood, mean antenna gain, } \\
\text { path loss, space-time correlations, } \\
\text { eigenvalue }\end{array}$ & $\mathrm{sC}$ \\
\hline [208], [209] & $\begin{array}{l}\text { Indoor AP } \\
\text { deployment }\end{array}$ & $\begin{array}{l}\text { Static and dynamic user movement, } \\
\text { LoS path }\end{array}$ & $\begin{array}{c}320-9570 \\
1-1080\end{array}$ & Deterministic & SISO & $\begin{array}{l}\text { outage, handoff, AP-AP distance, } \\
\text { path loss, required power, cell } \\
\text { packing, repeater assistance, } k \text {-factor, } \\
\text { spectral efficiency, antenna beamwidth, } \\
\text { cell radius, spreading loss, } \\
\text { molecular absorption, capacity }\end{array}$ & - \\
\hline $\begin{array}{l}{[210][211],} \\
{[213],[214]}\end{array}$ & $\begin{array}{l}\text { T2I/inside-station, } \\
\text { Intra-wagon, I2I }\end{array}$ & LoS, light-NLoS,deep-NLoS & $300-308$ & $\begin{array}{l}\text { quasi-deterministic, } \\
\text { calibrated RT }\end{array}$ & SISO & $\begin{array}{c}\text { path loss, shadow fading, } \\
\text { RMS delay spread, Rician K-factor, } \\
\text { azimuth/elevation angular spread } \\
\text { of arrival/departure, channel gain } \\
\text { cross-polarization ratio, cross correlations } \\
\text { capacity }\end{array}$ & $\mathrm{sC}$ \\
\hline [222] & ISL & LoS & $100-10000$ & GBSM & SISO & $\begin{array}{l}\text { Polarization and frequency diversity, } \\
\text { beam misalignment loss, link } \\
\text { capacity, solar wind effects, attenuation } \\
\text { and absorption by atmosphere, rain, } \\
\text { cloud and fog, plasma frequency, electron } \\
\text { density }\end{array}$ & - \\
\hline [223] & ISL & LoS & - & Theoretical & SISO & $\begin{array}{l}\text { Doppler wavelength shift, time delay } \\
\text { number of laser link hops }\end{array}$ & - \\
\hline [224] & ISL & LoS & - & $\begin{array}{l}\text { Stochastic network } \\
\text { calculus }\end{array}$ & SISO & $\begin{array}{l}\text { Doppler frequency shift, noise, delay } \\
\text { and backlog performance, Rician fading }\end{array}$ & - \\
\hline
\end{tabular}




\begin{tabular}{|c|c|c|c|c|c|c|c|}
\hline Ref & Application & Scenario & Freq. (GHz) & Modeling approach & Antenna & Channel Statistics & $\begin{array}{l}\text { Measurement Cam- } \\
\text { paign }\end{array}$ \\
\hline [225] & ISL & LoS & - & deterministic & SISO & $\begin{array}{l}\text { Polarization diversity, Q-factor, BER, } \\
\text { eye opening, received power }\end{array}$ & \\
\hline [230] & $\mathrm{V} 2 \mathrm{~V}$ & LoS, reflection, scattering & 300 & $\begin{array}{l}\text { deterministic, } \\
\text { stochastic }\end{array}$ & SISO & $\begin{array}{l}\text { Multipath interference, SNR, link } \\
\text { capacity, SIR, reflection and } \\
\text { scattering losses }\end{array}$ & THZ-TDS \\
\hline [232] & $\mathrm{V} 2 \mathrm{~V}$ & LoS, reflection, penetration & 300 & NGBSM & SISO & $\begin{array}{c}\text { Reflection and penetration losses, } \\
\text { path loss, AoD, AoA }\end{array}$ & $\mathrm{SC}$ \\
\hline [231] & $\mathrm{V} 2 \mathrm{~V}$ & $\begin{array}{c}\text { LoS, side reflection, rear } \\
\text { refletion, under-vehicle propagation, } \\
\text { blockage }\end{array}$ & 300 & NGBSM & $\begin{array}{l}4 \times 4 \text { MIMO, } \\
\text { SISO }\end{array}$ & $\begin{array}{l}\text { Path loss, path gain, AoA, AoD, ToA, } \\
\text { delay spread, angular spread }\end{array}$ & $\mathrm{SC}$ \\
\hline [235] & V2I & LoS, NLoS & 110 & $\begin{array}{l}\text { RT, time-varying } \\
\text { GBSM }\end{array}$ & SISO & $\begin{array}{c}\text { Path loss, ToA, DoA, correlation } \\
\text { probability, delay spread, MPC Birth-death } \\
\text { process anaysis. }\end{array}$ & - \\
\hline [236] & $\begin{array}{c}\text { intra-vehicle, } \\
\text { inter-vehicle, } \\
\text { vehicle-to-roadside }\end{array}$ & LoS, reflection, scattering & $\begin{array}{l}60-1000 \\
300-400 \\
800-900\end{array}$ & RT & SISO & $\begin{array}{l}\text { AoA, AoD, Capacity, path gain, } \\
\text { delay spread, coherence bandwidth }\end{array}$ & - \\
\hline [234] & V2I & LoS, NLoS & $300-308$ & Calibrated-RT & SISO & $\begin{array}{l}\text { Path loss, Rician k-factor, AoA, AoD } \\
\text { delay spread, angular spread, rain, snow }\end{array}$ & $\mathrm{SC}$ \\
\hline [240] & D2D desktop & $\begin{array}{l}\text { LoS, NLoS, single-reflection, } \\
\text { double-reflection }\end{array}$ & $0-60$ & GBSM & SISO & ToA, frequency correlation function & - \\
\hline [241] & Nano-network & LoS & $0-10000$ & Stochastic & SISO & $\begin{array}{l}\text { Path loss, molecular absorption noise, } \\
\text { capacity, power allocation, radiative } \\
\text { transfer theory }\end{array}$ & - \\
\hline$[252]$ & D2D Urban & LoS & $140-141$ & NGBSM & SISO & path loss, delay spread, angular spread & VNA \\
\hline [243] & iWNSN & LoS, scattering & $100-10000$ & Stochastic & SISO & $\begin{array}{l}\text { Intra-body Spreading, } \\
\text { molecular absorption, } \\
\text { scattering loss by human tissue }\end{array}$ & - \\
\hline [244] & Intra-body & LoS & $500-1500$ & $\begin{array}{c}\text { Deterministic, } \\
\text { stochastic }\end{array}$ & SISO & $\begin{array}{l}\text { Capacity, path loss related to } \\
\text { frequency, distance and dielectric } \\
\text { loss of human tissue }\end{array}$ & - \\
\hline [245] & Intra-body & LoS & $500-1500$ & $\begin{array}{c}\text { Deterministic, } \\
\text { stochastic }\end{array}$ & SISO & $\begin{array}{l}\text { Tissue absorption, spreading loss, error } \\
\text { prediction between model and data }\end{array}$ & THZ-TDS \\
\hline [246] & iWNSN & LoS, scattering & - & Stochastic & SISO & $\begin{array}{c}\text { Johnson-Nyquist noise, } \\
\text { black body noise, } \\
\text { Doppler shift noise, total noise } \\
\text { power spectral density } \\
\end{array}$ & - \\
\hline [247] & In-cell & LoS, NLoS & 4000-10000 & Deterministic & SISO & $\begin{array}{c}\text { path loss, reflection loss, } \\
\text { giant optical activity, } \\
\text { chirality effects, power allocation }\end{array}$ & - \\
\hline [249] & Indoor & $\begin{array}{l}\text { LoS, NLoS, scattering, } \\
\text { diffraction }\end{array}$ & $60-10000$ & $\begin{array}{l}\text { Theoretical, } \\
\text { empirical }\end{array}$ & SISO & $\begin{array}{l}\text { path gain, AoA, ToA, } \\
\text { delay spread, coherence bandwidth, } \\
\text { channel capacity, path loss, } \\
\text { broadening factor }\end{array}$ & - \\
\hline$[250]$ & Indoor & LoS, NLoS, scattering & $100-10000$ & Deterministic & SISO & Path loss, capacity & - \\
\hline [251] & General & LoS, reflected path & $0-2000$ & $\begin{array}{l}\text { Theoretical, } \\
\text { empirical }\end{array}$ & SISO & $\begin{array}{c}\text { Fading amplitude, coherence } \\
\text { bandwidth, roughness standard } \\
\text { deviation, absorption coefficient, } \\
\text { transmittance, delay, linear phase } \\
\end{array}$ & - \\
\hline [252] & Indoor/D2D & LoS, NLoS, scattering & $300-350$ & GBSM & UM-MIMO & $\begin{array}{c}\text { angular spread, delay spread, } \\
\text { space-time-frequency non-stationarities, } \\
\text { cluster angle-power- } \\
\text { delay, cluster angular spread, delay spread }\end{array}$ & - \\
\hline [254] & $\begin{array}{l}\text { Rough surface } \\
\text { Characterization }\end{array}$ & $\begin{array}{l}\text { Back-scattering for various } \\
\text { grit levels }\end{array}$ & 300 & Empirical & SISO & $\begin{array}{l}\text { Backscattering coefficient, } \\
\text { received power, RMS of surface } \\
\text { roughness, EM roughness, relative } \\
\text { permittivity, polarization varying }\end{array}$ & THz-TDS \\
\hline [255] & $\begin{array}{l}\text { Rough surface } \\
\text { characterization }\end{array}$ & $\begin{array}{l}\text { Backscattering for various } \\
\text { grit levels }\end{array}$ & $79,300,680$ & Empirical & SISO & $\begin{array}{l}\text { RMS of surface roughness, } \\
\text { scattering coefficients, } \\
\text { EM roughness } \\
\end{array}$ & VNA \\
\hline [256] & $\begin{array}{c}\text { Rough surface } \\
\text { characterization }\end{array}$ & $\begin{array}{l}\text { Metallic surface reflection } \\
\text { spectra }\end{array}$ & $300-3000$ & $\begin{array}{c}\text { Theoretical, } \\
\text { empirical }\end{array}$ & SISO & $\begin{array}{c}\text { Reflectance, RMS roughness, } \\
\text { fitting coefficient }\end{array}$ & $\begin{array}{l}\text { Spectroscopy, stylus } \\
\text { surface profilometer }\end{array}$ \\
\hline [257] & $\begin{array}{c}\text { Rough surface } \\
\text { characterization }\end{array}$ & Scattering & 400,650 & $\begin{array}{c}\text { Theoretical, } \\
\text { empirical }\end{array}$ & SISO & Conductivity & $\begin{array}{c}\text { Hemispherical quasi- } \\
\text { optical resonator }\end{array}$ \\
\hline [41] & $\begin{array}{l}\text { Rough surface } \\
\text { characterization }\end{array}$ & Reflection, scattering & $100-1000$ & $\begin{array}{c}\text { GBSM, } \\
\text { deterministic }\end{array}$ & SISO & $\begin{array}{l}\text { Absorption coefficient, } \\
\text { refractive index, reflection } \\
\text { coefficient calculations }\end{array}$ & THz-TDS \\
\hline$[42]$ & $\begin{array}{c}\text { Rough surface } \\
\text { characterization }\end{array}$ & Reflection, diffuse scattering & $100-4000$ & Empirical & SISO & Path gain, AoA, AoD & THz-TDS \\
\hline [43] & $\begin{array}{c}\text { Rough surface } \\
\text { characterization }\end{array}$ & Reflection, diffuse scattering & $60-4000$ & Empirical & SISO & Fading amplitude, ToA & THz-TDS \\
\hline
\end{tabular}

advances in the beam tracking and beam-steering capabilities and phased array technologies at $\mathrm{THz}$, we may be able to capture these extremely sensitive channel parameters, given that proper channel sounder architecture enables us to capture this information extremely fast in real-time by generating thousands of PDPs every second and over large bandwidths that is limiting factor when it comes to dynamic channel sounding architecture.

4) $\boldsymbol{R} \boldsymbol{F}$ EMF Exposure: The International Commission on Non-Ionizing Radiation Protection (ICNIRP) has so far defined exposure guidelines up to $300 \mathrm{GHz}$ [266]. Subsequently, it becomes a case of what happens beyond that range that will allow limits to be set. $\mathrm{THz}$ radiation is generally considered as a form of non-ionizing radiation due to the low photon energy ( 0.1 to $12.4 \mathrm{meV}$ compared to $12 \mathrm{eV}$ required for an electron to be released from an atom for radiation to become ionizing [267], [268], [269]), making it far less likely to cause health hazards in the form of gene mutations and illnesses such as cancer. In fact, it can be expected that if you point a $\mathrm{THz}$ source towards e.g., a human hand and the power levels are sufficiently large i.e.1 Watt it would actually hurt causing a burning sensation. Recent studies however, have shown that with high intensity transmission, different biological molecules exhibit absorption spectra inducing resonances that may damage but also repair DNA and protein cells [270], [271]. Research is still at its early stages and due to the lack of measurement data we are unable to determine $\mathrm{THz}$ health effects to establish radiation limits caused by short-term and long-term exposure. Furthermore, exposure effects on the body may be different, more in regard to where the devices may be used on the body, such as in smart glasses/watches that then could directly couple or radiate to the skin and eyes. Therefore, it is extremely important to be able to understand the health effects by appropriate modeling at a molecular level to understand the biologica to enable guidelines to be written indicating exposure limits for safe use of $\mathrm{THz}$ radiation. 


\section{B. Future Research Directions}

1) UM-MIMO Channel Modeling: As the wavelength is extremely small at $\mathrm{THz}$ frequencies, antenna arrays with a large number of elements can be placed compactly in a small amount of space to form Ultra-Massive Multiple-Input Multiple-Output (UM-MIMO) that could potentially tackle the distance problem [272]. The spectral efficiency of cellular networks can be improved by the unprecedented array gain and spatial multiplexing offered by UM-MIMO which can offer the capability of increased data rates and capacity [273]. The concept of UM-MIMO with 1024 antennas placed on the Tx and Rx side respectively was introduced in [274] and could be achieved by the use of plasmonic meta-materials [275], [276] that could form nano-antenna Array of Sub-Arrays (AoSA) architectures [277] which will be used to tackle the distance problem and allow high data rate links for communication distances of several tens of meters, while the first efforts in developing a model as such were presented in [278], [279]. Existing models are not adequate to characterize the UMMIMO channel efficiently and accurately and therefore there is a large gap in literature when is comes to modeling these sorts of channels. Models developed would have to consider the isolation between the antenna elements and antenna array configuration. As the amount of antennas increases the effects of diffuse scattering will have a big impact on the spatial and temporal dispersion at $\mathrm{THz}$ [115]. The huge amount of data that would have to be processed resulting from the large number of specular and diffuse reflections and the resulting clustering behavior that are inevitable at $\mathrm{THz}$ would lead to massive computational resource requirements that may require utilization of AI methods to enable precise and efficient characterization of the propagation channel. Furthermore, as the array dimensions grow larger, the plane wave assumption may no longer be valid for rough surface scattering characterization, and estimating the AoA of different MPCs would become extremely difficult. Alternatively, the spherical wave model [280], [281] may be applied instead to improve the accuracy of the estimated results [282], [283].

2) Material Characterization: The ITU-R P.1238-10 recommendation [284] models the effects of building materials on radio wave propagation for indoor systems and WLANs up to $450 \mathrm{GHz}$; Though these models are not validated by measurement, something that has only been done up to 100 $\mathrm{GHz}$ based on the ITU-R P.1238-7 recommendation [285]. Nevertheless, in [286] simulations for in-office and in-street scenarios were conducted for the $90-200 \mathrm{GHz}$ frequency range that consider material properties such as permittivity and conductivity and aim to extend the applicability of the ITU model for characterization of these materials up to $200 \mathrm{GHz}$. Determining material parameters can assist in characterizing the propagation channel in terms of absorption losses and multipath scattering behaviour. Parameter extraction methods used in the lower microwave bands, such as waveguides [287] and resonant cavities [288] are no longer a viable option due to problems related to the much smaller dimensions at THz. One interesting method that can be used to obtain these parameters is by using calibrated RT simulators fed by experimental data where extensive simulations could be used along with different algorithms [289], [290] to extract material parameters such as the dielectric constant. The experimental data can be collected through simple free space reflection and transmission as well as only transmission measurements using a VNA, though each method has its own related uncertainty to be accounted for when it comes to parameter extraction [291].

3) AI enabled Channel Modeling: The overwhelming growth of wireless data is pushing towards the limits of communications system performance. This exponential growth in wireless data poses as a challenge and future wireless communications systems will require powerful algorithms for different services. The applications of Artificial Intelligence (AI) in the field of wireless communications is receiving a lot of attention and the use of Machine Learning (ML) as an application of AI are currently being explored for radio propagation prediction [292], [293], [294]. As such, An AI driven approach by reinforcement learning was presented in [295] for periodic AoA probability estimation by feeding estimates from previous states to the algorithm for indoor scenarios, while RT and human movement modeling are used to validate the accuracy of the algorithm. Moreover, a possible data mining technique that can be employed for radio channel characterization involves the use of Support Vector Machines (SVMs). SVM is a supervised learning model that is based on finding the optimal position of hyper-planes in an $N$ dimensional space that separates the data points into different classes and has been employed in antenna design parameter prediction [296] as well as for clustering analysis [297].

The existing channel modeling techniques, either deterministic or measurement based have certain challenges and difficulties that make it hard for us to make an accurate characterization of the propagation channel. When it comes to deterministic models, long simulation times and high number of computational resources especially with larger bandwidths and increasing number of antennas, make the modeling of the channel at $\mathrm{THz}$ very difficult. Measurement based techniques on the other hand, such as GBSM and NGBSM would require a great amount of empirical data that would take too long to obtain as it is very difficult to characterize the channel at every point in space at these larger bandwidths using the current channel sounding techniques; hence, these models use limited sets of data leading to less accurate characterization. New ways need to be explored that would allow much more efficient and detailed analysis of the data so that the accuracy can be improved and at the same time simulation times can decrease.

AI enabled channel modeling requires a large amount of data as input to the algorithms. This data is used to train these algorithms and make predictions. In this way the computational complexity can be greatly reduced as long as the predictions made are accurate and the algorithms are robust for modeling different scenarios. The disadvantages posed by hybrid deterministic-stochastic models in regards to simulation time and limited channel sounding prohibit large scale characterization. At THz particularly, small-scale interactions due to the short wavelength $(<1 \mathrm{~mm})$ above $300 \mathrm{GHz}$ are relevant and cannot be modeled efficiently over large bandwidths with 
the existing techniques.

4) Hybrid Channel Modeling: It is apparent that the hybrid modeling approach where empirical data are fed to RT simulators to extend the limited data sets is very popular. Similarly, we believe that channel characterization should be expanded and complemented by the use of various stochastic and deterministic models to describe each part of signal propagation from one end of the communications system to the other. As an example, many empirical data sets can be collected and an SV type model can be used for grouping MPCs together and a second order GMM can be used to describe MPCs within arriving clusters. Furthermore, FDTD/MOM modeling can be employed to model a portion of the propagating environment when RT simulations do not provide enough information about the channel. It provides much higher accuracy and manages computational complexity since it can be applied to model areas of complex discontinuities [298]. These methods can be then tuned within a unified model, in parallel with great knowledge of the weather conditions and scatterer properties, and employed along with ML techniques that could perhaps allow for this characterization to be expanded over larger bandwidths due to technological limitations of real-time channel sounders which are preferred for collecting data, but are limited by their short bandwidths.

5) Improved Channel Sounding: Channel sounding at $\mathrm{THz}$ within a 3D environment allows for a spatial and temporal analysis that can characterize and predict the behaviour of wave propagation in terms of ToA, AoA and Doppler shifts. Good knowledge of these parameters enables prediction of a communications systems performance and highly influences standardisation processes. The impact directive antennas have in dynamic scenarios have a major impact on system performance, and the lack of experimental knowledge at $\mathrm{THz}$ frequencies draws the need for new measurement procedures for correct system evaluation and design. Different sounder architectures will have to be developed and compared to improve the estimation accuracy and enhance the bandwidth. A proper model has to be then developed that is calibrated with measurement data which will allow for estimates independent of channel sounding device. Phased arrays and their behaviour when sounding the channel with wideband signals in terms of mutual coupling between antenna elements, near field effects, the effects of non-linear distortions as well as uncertainties in the measurement procedures, are big challenges that need to be correctly investigated to achieve a high degree of accuracy in parameter estimation.

Moreover, channel characteristics that are vital in system design such as delay and Doppler spread, coherence time and bandwidth are heavily dependent on the spatial characteristic of the antenna, which acts as a spatial filter due to its high directivity. This means that channel characteristics and system design including modulator design, symbol structure and RF front end design become largely dependent on one another. Therefore, to perform channel measurements at $\mathrm{THz}$, all parts of communication system design need to be characterized and evaluated to optimize the performance and enable accurate high data rate measurements in order to realize $\mathrm{THz}$ communications, and that can be done by tweaking the performance of each component individually and measuring their effects on the system's BER [299]. Future 6G systems will need these capabilities that are still in their early stages at $\mathrm{THz}$.

\section{CONCLUSIONS}

This paper provided an overview of measurements and modeling of the propagation channel at THz. It begins with an introduction to the $\mathrm{THz}$ band stating what applications could be realized and discusses the $\mathrm{THz}$ integrated systems evolution and provides information of the standards being developed. THz propagation characteristics such as FSPL, meteorological attenuation and diffuse scattering were further introduced. Measurement requirements along with $\mathrm{THz}$ channel sounding methods were then described based on the applications identified. Afterward, an extensive review of the state of the art measurements and modeling in the $\mathrm{THz}$ bands was presented along with useful information about the requirements for each application scenario. Finally, an insight was given to the issues and challenges faced at modeling in the THz spectrum as well as the ongoing research opportunities that are being realized to tackle the challenges faced for communication systems modeling at THz. This paper provides a solid understanding of channel propagation phenomena, measurement techniques and modeling at $\mathrm{THz}$ and gives a good knowledge base for future work to be conducted to realize various applications targeted for operation at this band.

\section{REFERENCES}

[1] Cherry, S. (2004). Edholm's law of bandwidth. IEEE Spectrum, 41(7), pp.58-60.

[2] R. Li, "Towards a new Internet for the year 2030 and beyond," in Proc. 3rd Annu. ITU IMT-2020/5G Workshop Demo Day, Geneva,Switzerland, 2018, pp. 1-21.

[3] C. Systems. (2019). Cisco Visual Networking Index: Global Mobile Data Traffic Forecast Update, White Paper 2017-2022. Accessed: Jan. 5, 2020. [Online]. Available: https://www.cisco.com/c/en/us/solutions/collateral/ serviceprovider/visual-networking-index-vni/white-paper-c11- 738429.html

[4] 2021. [Online]. Available: https://www.ericsson.com/en/mobilityreport/dataforecasts/mobile-traffic-forecast.

[5] "Radio Spectrum Allocation", Federal Communications Commission, 2020. [Online]. Available: http://www.fcc.gov/oet/spectrum/.

[6] S. Dang, O. Amin, B. Shihada, and M.-S. Alouini. What should $6 \mathrm{~g}$ be? Nature Electronics, 3(1):2520-1131, 2020

[7] Ian F. Akyildiz, Josep Miquel Jornet, Chong Han, Terahertz band: Next frontier for wireless communications, Physical Communication, Volume 12, 2014, Pages 16-32, ISSN 1874-4907, $\quad$ https://doi.org/10.1016/j.phycom.2014.01.006. (https://www.sciencedirect.com/science/article/pii/S1874490714000238)

[8] H. Song and T. Nagatsuma, "Present and Future of Terahertz Communications", IEEE Transactions on Terahertz Science and Technology, vol. 1, no. 1, pp. 256-263, 2011. Available: 10.1109/tthz.2011.2159552.

[9] M. Latva-aho and K. Leppanen, "Key drivers and research challenges for " 6G ubiquitous wireless intelligence (white paper)," 6G Flagship research program, University of Oulu, Finland, 2019.

[10] C. Castro, "6G Gains Momentum With Initiatives Launched Across the World", 6GWorld, 2021. [Online]. Available: https://www.6gworld.com/exclusives/6g-gains-momentum-withinitiatives-launched-across-the-world/.

[11] "Unlocking the potential of Terahertz radio spectrum", Ofcom.org.uk, 2021. [Online]. Available: https://www.ofcom.org.uk/_data/assets/pdf_file/0032/228929/terahertzspectrum-paper.pdf. [Accessed: 26- Dec- 2021].

[12] S. Chinchali, A. Sharma, J. Harrison, A. Elhafsi, D. Kang, E. Pergament, E. Cidon, S. Katti, M. Pavone, "Network offloading policies for cloud robotics: A learning-based approach," Feb. 2019, arXiv:1902.05703. [Online]. Available: https://arxiv.org/abs/1902.05703 
[13] ] S. Garg et al., "Enabling the next generation of mobile robotics using 5G wireless," IEEE Access, submitted for publication.

[14] M. Chen, Y. Tian, G. Fortino, J. Zhang, and I. Humar, "Cognitive Internet of vehicles," Comput. Commun., vol. 120, pp. 58-70, May 2018.

[15] M. Tonouchi, "Cutting-edge terahertz technology," Nature Photon., vol. 1, no. 2, pp. 97-105, Feb. 2007.

[16] D. M. Mittleman, R. H. Jacobsen, R. Neelamani, R. G. Baraniuk, and M. C. Nuss, "Gas sensing using terahertz time-domain spectroscopy," Appl. Phys. B, vol. 67, no. 3, pp. 379-390, Sep. 1998.

[17] M. Aladsani, A. Alkhateeb, and G. C. Trichopoulos, "Leveraging mmWave imaging and communications for simultaneous localization and mapping," in Proc. IEEE Int. Conf. Acoust., Speech Signal Process. (ICASSP), May 2019, pp. 4539-4543.

[18] M. J. W. Rodwell, Y. Fang, J. Rode, J. Wu, B. Markman, S. T. Š. Brunelli, J. Klamkin, and M. Urteaga, "100-340 GHz systems: Transistors and applications," in IEDM Tech. Dig., Dec. 2018, pp. 14.3.1-14.3.4.

[19] S. Koenig, D. Lopez-Diaz, J. Antes, F. Boes, R. Henneberger, A. Leuther, A. Tessmann, R. Schmogrow, D. Hillerkuss, R. Palmer, T. Zwick, C. Koos, W. Freude, O. Ambacher, J. Leuthold, and I. Kallfass, "Wireless sub-THz communication system with high data rate," Nature Photon., vol. 7, pp. 977-981, Oct. 2013.

[20] T. S. Rappaport, "6G and beyond: Terahertz communications and sensing," 2019 Brooklyn 5G Summit Keynote, Apr. 2019. [Online]. Available: https://ieeetv.ieee.org/conference-highlights/keynotetedrappaport-terahertz-communication-b5gs-2019?

[21] M. Aladsani, A. Alkhateeb, and G. C. Trichopoulos, "Leveraging mmWave imaging and communications for simultaneous localization and mapping," in Proc. IEEE Int. Conf. Acoust., Speech Signal Process. (ICASSP), May 2019, pp. 4539-4543.

[22] O. Kanhere and T. S. Rappaport, "Position locationing for millimeter wave systems," in Proc. IEEE Global Commun. Conf., Dec. 2018, pp. 206-212.

[23] J. Federici and L. Moeller, "Review of terahertz and subterahertz wireless communications", Journal of Applied Physics, vol. 107, no. 11, p. 111101, 2010. Available: 10.1063/1.3386413.

[24] I. Akyildiz, J. Jornet and C. Han, "TeraNets: ultra-broadband communication networks in the terahertz band", IEEE Wireless Communications, vol. 21, no. 4, pp. 130-135, 2014. Available: 10.1109/mwc.2014.6882305

[25] Z. Chen, et al., "A survey on terahertz communications," China Communications, vol. 16, no. 2, pp. 1-35, Feb. 2019.

[26] M. Kemp, P. Taday, B. Cole, J. Cluff, A. Fitzgerald and W. Tribe, "Security applications of terahertz technology", Terahertz for Military and Security Applications, 2003. Available: 10.1117/12.500491.

[27] T. Schneider, A. Wiatrek, S. Preussler, M. Grigat and R. Braun, "Link Budget Analysis for Terahertz Fixed Wireless Links," in IEEE Transactions on Terahertz Science and Technology, vol. 2, no. 2, pp. 250-256, March 2012, doi: 10.1109/TTHZ.2011.2182118

[28] I. Malhotra, K. Jha and G. Singh, "Terahertz antenna technology for imaging applications: a technical review", International Journal of Microwave and Wireless Technologies, vol. 10, no. 3, pp. 271-290, 2018. Available: $10.1017 / \mathrm{s} 175907871800003 x$.

[29] He, D., Guan, K., Fricke, A., Ai, B., He, R., Zhong, Z., Kasamatsu, A., Hosako, I. and Kurner, T. (2017). Stochastic Channel Modeling for Kiosk Applications in the Terahertz Band. IEEE Transactions on Terahertz Science and Technology, 7(5), pp.502-513.

[30] C. Cheng and A. Zajić, "Characterization of Propagation Phenomena Relevant for $300 \mathrm{GHz}$ Wireless Data Center Links," in IEEE Transactions on Antennas and Propagation, vol. 68, no. 2, pp. 1074-1087, Feb. 2020, doi: 10.1109/TAP.2019.2949135.

[31] J. Fu, P. Juyal and A. Zajić, "THz Channel Characterization of Chipto-Chip Communication in Desktop Size Metal Enclosure," in IEEE Transactions on Antennas and Propagation, vol. 67, no. 12, pp. 75507560, Dec. 2019, doi: 10.1109/TAP.2019.2934908.

[32] B. K. Jung, N. Dreyer, J. M. Eckhard and T. Kürner, "Simulation and Automatic Planning of $300 \mathrm{GHz}$ Backhaul Links," 2019 44th International Conference on Infrared, Millimeter, and Terahertz Waves (IRMMW-THz), Paris, France, 2019, pp. 1-3, doi: 10.1109/IRMMWTHz.2019.8873734.

[33] K. Guan et al., "On Millimeter Wave and THz Mobile Radio Channel for Smart Rail Mobility," in IEEE Transactions on Vehicular Technology, vol. 66, no. 7, pp. 5658-5674, July 2017, doi: 10.1109/TVT.2016.2624504.
[34] I. Mehdi, J. Siles, C. P. Chen, and J. M. Jornet, "Thz technology for space communications," in 2018 Asia-Pacific Microwave Conference (APMC), Nov 2018, pp. 76-78.

[35] "Terahertz Communication for Vehicular Networks," in IEEE Transactions on Vehicular Technology, vol. 66, no. 7, pp. 5617-5625, July 2017, doi: 10.1109/TVT.2017.2712878.

[36] F. Jameel, Z. Hamid, F. Jabeen, S. Zeadally and M. A. Javed, "A Survey of Device-to-Device Communications: Research Issues and Challenges," in IEEE Communications Surveys \& Tutorials, vol. 20, no. 3 , pp. 2133-2168, thirdquarter 2018, doi: 10.1109/COMST.2018.2828120.

[37] J. O'Hara, S. Ekin, W. Choi and I. Song, "A Perspective on Terahertz Next-Generation Wireless Communications", Technologies, vol. 7, no. 2, p. 43, 2019. Available: 10.3390/technologies7020043.

[38] K. Humphreys et al., "Medical applications of terahertz imaging: a review of current technology and potential applications in biomedical engineering", The 26th Annual International Conference of the IEEE Engineering in Medicine and Biology Society. Available: 10.1109/iembs.2004.1403410.

[39] S. Ghafoor, N. Boujnah, M. H. Rehmani and A. Davy, "MAC Protocols for Terahertz Communication: A Comprehensive Survey," in IEEE Communications Surveys \& Tutorials, vol. 22, no. 4, pp. 2236-2282, Fourthquarter 2020, doi: 10.1109/COMST.2020.3017393.

[40] E. Nichols, "A Study of the Transmission Spectra of Certain Substances in the Infra-Red", Physical Review (Series I), vol. 1, no. 1, pp. 1-18, 1893. Available: $10.1103 /$ physrevseriesi.1.1.

[41] R. Piesiewicz, C. Jansen, D. Mittleman, T. Kleine-Ostmann, M. Koch and T. Kurner, "Scattering Analysis for the Modeling of $\mathrm{THz}$ Communication Systems," in IEEE Transactions on Antennas and Propagation, vol. 55, no. 11, pp. 3002-3009, Nov. 2007, doi: 10.1109/TAP.2007.908559.

[42] J. Kokkoniemi, V. Petrov, D. Moltchanov, J. Lehtomaeki, Y. Koucheryavy and M. Juntti, "Wideband Terahertz Band Reflection and Diffuse Scattering Measurements for Beyond 5G Indoor Wireless Networks," European Wireless 2016; 22th European Wireless Conference, Oulu, Finland, 2016, pp. 1-6.

[43] J. Kokkoniemi, J. Lehtomäki and M. Juntti, "Measurements on rough surface scattering in terahertz band," 2016 10th European Conference on Antennas and Propagation (EuCAP), Davos, 2016, pp. 1-5, doi: 10.1109/EuCAP.2016.7481175.

[44] Sengupta, K., Nagatsuma, T. \& Mittleman, D.M. Terahertz integrated electronic and hybrid electronic-photonic systems. Nat Electron 1, 622-635 (2018). https://doi.org/10.1038/s41928-018-0173-2

[45] R. Lewis, "A review of terahertz detectors", Journal of Physics D: Applied Physics, vol. 52, no. 43, p. 433001, 2019. Available: 10.1088/1361-6463/ab31d5.

[46] R. Lewis, "A review of terahertz sources", Journal of Physics D: Applied Physics, vol. 47, no. 37, p. 374001, 2014. Available: 10.1088/0022-3727/47/37/374001

[47] R. Bogue, "Sensing with terahertz radiation: a review of recent progress", Sensor Review, vol. 38, no. 2, pp. 216-222, 2018. Available: 10.1108/sr-10-2017-0221.

[48] A. Maestrini et al., "Frequency tunable electronic sources working at room temperature in the 1 to $3 \mathrm{THz}$ band", SPIE Proceedings, 2012. Available: $10.1117 / 12.929654$

[49] I. Mehdi, J. V. Siles, C. Lee and E. Schlecht, "THz Diode Technology: Status, Prospects, and Applications," in Proceedings of the IEEE, vol. 105, no. 6, pp. 990-1007, June 2017, doi: 10.1109/JPROC.2017.2650235.

[50] K. Leong et al., "A $0.85 \mathrm{THz}$ Low Noise Amplifier Using InP HEMT Transistors", IEEE Microwave and Wireless Components Letters, vol. 25, no. 6, pp. 397-399, 2015. Available: 10.1109/lmwc.2015.2421336.

[51] M. Asada and S. Suzuki, "Terahertz Emitter Using Resonant-Tunneling Diode and Applications," Sensors, vol. 21, no. 4, p. 1384, Feb. 2021

[52] S. Yang and M. Jarrahi, "High-power continuous-wave terahertz generation through plasmonic photomixers," 2016 IEEE MTT-S International Microwave Symposium (IMS), 2016, pp. 1-4, doi: 10.1109/MWSYM.2016.7540333

[53] S. Yang et al., "A compact and broadly tunable terahertz source based on a bimodal laser and plasmonic photomixer," 2016 41st International Conference on Infrared, Millimeter, and Terahertz waves (IRMMWTHz), 2016, pp. 1-2, doi: 10.1109/IRMMW-THz.2016.7758608.

[54] C. Walther, M. Fischer, G. Scalari, R. Terazzi, N. Hoyler and J. Faist, "Quantum cascade lasers operating from 1.2to1.6THz", Applied Physics Letters, vol. 91, no. 13, p. 131122, 2007. Available: 10.1063/1.2793177. 
[55] Lu, Q., Wu, D., Sengupta, S. et al. Room temperature continuous wave, monolithic tunable $\mathrm{THz}$ sources based on highly efficient mid-infrared quantum cascade lasers. Sci Rep 6, 23595 (2016). https://doi.org/10.1038/srep23595

[56] S. Hayashi, K. Nawata, Y. Takida, Y. Tokizane, K. Kawase and H. Minamide, "High-Brightness Continuously Tunable Narrowband Subterahertz Wave Generation," in IEEE Transactions on Terahertz Science and Technology, vol. 6, no. 6, pp. 858-861, Nov. 2016, doi: 10.1109/TTHZ.2016.2611939.

[57] Yasuaki Monnai, Kristian Altmann, Christian Jansen, Hartmut Hillmer, Martin Koch, and Hiroyuki Shinoda, "Terahertz beam steering and variable focusing using programmable diffraction gratings," Opt. Express 21, 2347-2354 (2013)

[58] Scherger, B., Reuter, M., Scheller, M. et al. Discrete Terahertz Beam Steering with an Electrically Controlled Liquid Crystal Device. J Infrared Milli Terahz Waves 33, 1117-1122 (2012). https://doi.org/10.1007/s10762-012-9927-5

[59] X. Deng, Y. Li, J. Li, C. Liu, W. Wu and Y. Xiong, "A 320$\mathrm{GHz} 1 \times 4$ Fully Integrated Phased Array Transmitter Using 0.13$\mu \mathrm{m}$ SiGe BiCMOS Technology," in IEEE Transactions on Terahertz Science and Technology, vol. 5, no. 6, pp. 930-940, Nov. 2015, doi: 10.1109/TTHZ.2015.2477604.

[60] Y. Yang, O. D. Gurbuz and G. M. Rebeiz, "An Eight-Element 370-410$\mathrm{GHz}$ Phased-Array Transmitter in 45-nm CMOS SOI With Peak EIRP of 8-8.5 dBm," in IEEE Transactions on Microwave Theory and Techniques, vol. 64 , no. 12, pp. 4241-4249, Dec. 2016, doi 10.1109/TMTT.2016.2613850

[61] H. Jalili and O. Momeni, "A 0.34-THz Wideband Wide-Angle 2-D Steering Phased Array in 0.13- $\mu$ m SiGe BiCMOS," in IEEE Journal of Solid-State Circuits, vol. 54, no. 9, pp. 2449-2461, Sept. 2019, doi: 10.1109/JSSC.2019.2925523.

[62] S. Rey, D. Ulm, T. Kleine-Ostmann and T. Kiirner, "Performance evaluation of a first phased array operating at $300 \mathrm{GHz}$ with horn elements," 2017 11th European Conference on Antennas and Propagation (EUCAP), Paris, 2017, pp. 1629-1633, doi: 10.23919/EuCAP.2017.7928868.

[63] B. Peng, S. Priebe, and T. Kürner, "Fast Beam Searching Concept for Indoor Terahertz Communications," in Proc. 8th European Conference on Antennas and Propagation (EUCAP), pp. 483-487, IEEE, 2014.

[64] B. Peng, K. Guan, S. Rey, T. Kürner, Two-Step Angle-of-Arrival Estimation for Terahertz Communications Based on Correlation of Power-Angular Spectra in Frequency, Proc. European Conference on Antennas and Propagation EuCAP 2018, London, March 2018, electronic publication, 5 pages

[65] C. Lin and G. Y. Li, "Adaptive Beamforming With Resource Allocation for Distance-Aware Multi-User Indoor Terahertz Communications," in IEEE Transactions on Communications, vol. 63, no. 8, pp. 2985-2995, Aug. 2015, doi: 10.1109/TCOMM.2015.2440356.

[66] "IEEE Standard for High Data Rate Wireless Multi-Media NetworksAmendment 2: $100 \mathrm{~Gb} / \mathrm{s}$ Wireless Switched Point-to-Point Physical Layer," in IEEE Std 802.15.3d-2017 (Amendment to IEEE Std 802.15.3-2016 as amended by IEEE Std 802.15.3e-2017), vol., no., pp.1-55, 18 Oct. 2017, doi: 10.1109/IEEESTD.2017.8066476.

[67] "IEEE Standard for High Data Rate Wireless Multi-Media Networks," in IEEE Std 802.15.3-2016 (Revision of IEEE Std 802.15.3-2003, vol., no., pp.1-510, 25 July 2016, doi: 10.1109/IEEESTD.2016.7524656.

[68] V. Petrov, T. Kurner and I. Hosako, "IEEE 802.15.3d: First Standardization Efforts for Sub-Terahertz Band Communications toward 6G," in IEEE Communications Magazine, vol. 58, no. 11, pp. 28-33, November 2020, doi: 10.1109/MCOM.001.2000273.

[69] T. Kürner and A. Hirata, "On the Impact of the Results of WRC 2019 on THz Communications," 2020 Third International Workshop on Mobile Terahertz Systems (IWMTS), Essen, Germany, 2020, pp. 1-3, doi: 10.1109/IWMTS49292.2020.9166206.

[70] FINAL ACTS WRC-12. ITU, 2012, p. 43.

[71] "Sharing and compatibility studies between land-mobile, fixed and passive services in the frequency range 275-450 GHz", Itu.int, 2019. [Online]. Available: https://www.itu.int/dms_pub/itu-r/opb/rep/R-REPSM.2450-2019-PDF-E.pdf.

[72] ] S. Rey, Initial results on sharing studies, Thor Deliverable D5.1; available at https://thorproject.eu/results/

[73] "World Radiocommunication Conference 2019 (WRC-19)", Itu.int, 2019. [Online]. Available: https://www.itu.int/en/ITUR/conferences/wrc/2019/Documents/PFA-WRC19-E.pdf.

[74] C. Wang, J. Bian, J. Sun, W. Zhang and M. Zhang, "A Survey of 5G Channel Measurements and Models," in IEEE Communications
Surveys \& Tutorials, vol. 20, no. 4, pp. 3142-3168, Fourthquarter 2018 , doi: 10.1109/COMST.2018.2862141.

[75] I. A. Hemadeh, K. Satyanarayana, M. El-Hajjar and L. Hanzo, "Millimeter-Wave Communications: Physical Channel Models, Design Considerations, Antenna Constructions, and Link-Budget," in IEEE Communications Surveys \& Tutorials, vol. 20, no. 2, pp. 870-913, Secondquarter 2018, doi: 10.1109/COMST.2017.2783541.

[76] T. S. Rappaport, G. R. MacCartney, M. K. Samimi and S. Sun, "Wideband Millimeter-Wave Propagation Measurements and Channel Models for Future Wireless Communication System Design," in IEEE Transactions on Communications, vol. 63, no. 9, pp. 3029-3056, Sept. 2015, doi: 10.1109/TCOMM.2015.2434384.

[77] Z. Chen et al., "A survey on terahertz communications," in China Communications, vol. 16, no. 2, pp. 1-35, Feb. 2019, doi: 10.12676/j.cc.2019.02.001

[78] H. Elayan, O. Amin, B. Shihada, R. M. Shubair and M. -S. Alouini, "Terahertz Band: The Last Piece of RF Spectrum Puzzle for Communication Systems," in IEEE Open Journal of the Communications Society, vol. 1, pp. 1-32, 2020, doi: 10.1109/OJCOMS.2019.2953633.

[79] C. -X. Wang, J. Huang, H. Wang, X. Gao, X. You and Y. Hao, "6G Wireless Channel Measurements and Models: Trends and Challenges," in IEEE Vehicular Technology Magazine, vol. 15, no. 4, pp. 22-32, Dec. 2020, doi: 10.1109/MVT.2020.3018436.

[80] Zhang, Jh., Tang, P., Yu, L. et al. Channel measurements and models for 6G: current status and future outlook. Front Inform Technol Electron Eng 21, 39-61 (2020). https://doi.org/10.1631/FITEE.1900450

[81] B. Peng, K. Guan, A. Kuter, S. Rey, M. Patzold and T. Kuerner, "Channel Modeling and System Concepts for Future Terahertz Communications: Getting Ready for Advances Beyond 5G," in IEEE Vehicular Technology Magazine, vol. 15, no. 2, pp. 136-143, June 2020 , doi: 10.1109/MVT.2020.2977014.

[82] C. Han and Y. Chen, "Propagation Modeling for Wireless Communications in the Terahertz Band," in IEEE Communications Magazine, vol. 56, no. 6, pp. 96-101, June 2018, doi: 10.1109/MCOM.2018.1700898.

[83] F. Lemic et al. (2019). Survey on Terahertz Nanocommunication and Networking: A Top-Down Perspective. [Online]. Available: https://arxiv.org/abs/1909.05703.

[84] Su, K., Moeller, L., Barat, R. B. \& Federici, J. F. Experimental comparison of terahertz and infrared data signal attenuation in dust clouds. J. Opt. Soc. Am. A 29, 2360-2366 (2012).

[85] L. Cang, H. Zhao and G. Zheng, "The Impact of Atmospheric Turbulence on Terahertz Communication," in IEEE Access, vol. 7, pp. 88685-88692, 2019, doi: 10.1109/ACCESS.2019.2925815.

[86] Internation Telecommunication Union. P.525: Calculation of free-space attenuation [Internet]. 2016. Available from: https://www.itu.int/rec/RREC-P.525/en

[87] T. Kleine-Ostmann and T. Nagatsuma, "A review on terahertz communications research," J. Infrared, Millimeter, Terahertz Waves, vol. 32, no. 2, pp. 143-171, 2011. doi: 10.1007/s10762-010-9758-1.

[88] Itu.int. 2021. [online] Available at: ¡https://www.itu.int/dms_pubrec/itur/rec/p/R-REC-P.676-10-201309-S!!PDF-E.pdf;

[89] Nagatsuma, T., Ducournau, G. \& Renaud, C. Advances in terahertz communications accelerated by photonics. Nature Photon 10, 371-379 (2016). https://doi.org/10.1038/nphoton.2016.65

[90] J. F. O'Hara and D. R. Grischkowsky, "Comment on the Veracity of the ITU-R Recommendation for Atmospheric Attenuation at Terahertz Frequencies," in IEEE Transactions on Terahertz Science and Technology, vol. 8, no. 3, pp. 372-375, May 2018, doi: 10.1109/TTHZ.2018.2814343.

[91] Y. Yang, M. Mandehgar and D. Grischkowsky, "Determination of water vapor continuum absorption, using long-path THz-TDS without the cross-term," 2014 39th International Conference on Infrared, Millimeter, and Terahertz waves (IRMMW-THz), Tucson, AZ, USA, 2014, pp. 1-2, doi: 10.1109/IRMMW-THz.2014.6956074.

[92] Gordon, I.E.; Rothman, L.S.; Hill, C.; Kochanov, R.V.; Tan, Y.; Bernath, P.F.; Birk, M.; Boudon, V.; Campargue, A.; Chance, K.; et al. The HITRAN2016 molecular spectroscopic database. J. Quant. Spectrosc. Radiat. Transf. 2017, 203, 3-69.

[93] Joonas Kokkoniemi, Janne Lehtomäki, Markku Juntti, A discussion on molecular absorption noise in the terahertz band, Nano Communication Networks, Volume 8, 2016, Pages 35-45, ISSN 1878-7789, https://doi.org/10.1016/j.nancom.2015.11.001. (https://www.sciencedirect.com/science/article/pii/S1878778915000472)

[94] Ma, J., Adelberg, J., Shrestha, R. et al. The Effect of Snow on a Terahertz Wireless Data Link. J Infrared Milli Terahz Waves 39, 505-508 (2018). https://doi.org/10.1007/s10762-018-0486-2 
[95] Itu.int. 2021. [online] Available at: ¡https://www.itu.int/dms_pubrec/itur/rec/p/R-REC-P.838-3-200503-I!!PDF-E.pdf $i$

[96] Rogalski, A., Sizov, F. Terahertz detectors and focal plane arrays. OptoElectron. Rev. 19, 346-404 (2011). https://doi.org/10.2478/s11772-0110033-3

[97] S. Saunders, Antennas and propagation for wireless communication systems. Chichester [etc.]: J. Wiley, 2005.

[98] Itu.int. 2021. [online] Available at: ;https://www.itu.int/dms_pubrec/itur/rec/p/R-REC-P.1817-1-201202-I!!PDF-E.pdf;

[99] E. Moon, T. Jeon and D. R. Grischkowsky, "Long-Path THz-TDS Atmospheric Measurements Between Buildings," in IEEE Transactions on Terahertz Science and Technology, vol. 5, no. 5, pp. 742-750, Sept. 2015, doi: 10.1109/TTHZ.2015.2443491.

[100] Itu.int. 2021. [online] Available at: ¡https://www.itu.int/dms_pubrec/itur/rec/p/R-REC-P.840-8-201908-I!!PDF-E.pdf

[101] J. Ma, R. Shrestha, L. Moeller, and D. M. Mittleman, "Invited article: Channel performance for indoor and outdoor terahertz wireless links," APL Photonics, vol. 3, no. 5, p. 051601, 2018.

[102] Itu.int. 2021. [online] Available at: ¡https://www.itu.int/dms_pubrec/itur/rec/p/R-REC-P.833-7-201202-S!!PDF-E.pdf;

[103] Gente, R., Rehn, A., Probst, T. et al. Outdoor Measurements of Leaf Water Content Using THz Quasi Time-Domain Spectroscopy. J Infrared Milli Terahz Waves 39, 943-948 (2018). https://doi.org/10.1007/s10762-018-0520-4.

[104] J. Kokkoniemi, J. Lehtomäki and M. Juntti, "Measurements on penetration loss in terahertz band," 2016 10th European Conference on Antennas and Propagation (EuCAP), Davos, Switzerland, 2016, pp. 1-5, doi: 10.1109/EuCAP.2016.7481176.

[105] H. Sawada et al., "Penetration loss measurement at $300 \mathrm{GHz}$ for building entry loss estimation," 12th European Conference on Antennas and Propagation (EuCAP 2018), London, UK, 2018, pp. 1-4, doi 10.1049/cp.2018.1158.

[106] Y. Xing and T. S. Rappaport, "Propagation Measurement System and Approach at $140 \mathrm{GHz}-$ Moving to 6G and Above $100 \mathrm{GHz}$,' 2018 IEEE Global Communications Conference (GLOBECOM), Abu Dhabi, United Arab Emirates, 2018, pp. 1-6, doi: 10.1109/GLOCOM.2018.8647921.

[107] T. S. Rappaport et al., "Wireless Communications and Applications Above $100 \mathrm{GHz}$ : Opportunities and Challenges for 6G and Beyond," in IEEE Access, vol. 7, pp. 78729-78757, 2019, doi: 10.1109/ACCESS.2019.2921522.

[108] V. Petrov, J. M. Eckhardt, D. Moltchanov, Y. Koucheryavy and T. Kurner, "Measurements of Reflection and Penetration Losses in Low Terahertz Band Vehicular Communications," 2020 14th European Conference on Antennas and Propagation (EuCAP), Copenhagen, Denmark, 2020, pp. 1-5, doi: 10.23919/EuCAP48036.2020.9135389.

[109] J. Kokkoniemi, P. Rintanen, J. Lehtomaki and M. Juntti, "Diffraction Effects in Terahertz Band - Measurements and Analysis," 2016 IEEE Global Communications Conference (GLOBECOM), Washington, DC, 2016, pp. 1-6, doi: 10.1109/GLOCOM.2016.7841734.

[110] D. Serghiou et al., "Comparison of Diffuse Roughness Scattering from Material Reflections at 500-750 GHz," 2021 15th European Conference on Antennas and Propagation (EuCAP), 2021, pp. 1-5, doi: 10.23919/EuCAP51087.2021.9411422.

[111] Alan H. Lettington, Isaiah M. Blankson, Magdy F. Attia, and Dennis Dunn "Review of imaging architecture", Proc. SPIE 4719, Infrared and Passive Millimeter-wave Imaging Systems: Design, Analysis, Modeling, and Testing, (29 July 2002); https://doi.org/10.1117/12.477457

[112] J. Ma, R. Shrestha, W. Zhang, L. Moeller and D. M. Mittleman, "Terahertz Wireless Links Using Diffuse Scattering From Rough Surfaces," in IEEE Transactions on Terahertz Science and Technology, vol. 9, no. 5, pp. 463-470, Sept. 2019, doi: 10.1109/TTHZ.2019.2933166.

[113] C. Jansen et al., "Diffuse Scattering From Rough Surfaces in $\mathrm{THz}$ Communication Channels," in IEEE Transactions on Terahertz Science and Technology, vol. 1, no. 2, pp. 462-472, Nov. 2011, doi: 10.1109/TTHZ.2011.2153610.

[114] G. R. Valenzuela, "Scattering of electromagnetic waves from a slightly rough surface moving with uniform velocity," in Radio Science, vol 3, no. 12, pp. 1154-1157, Dec. 1968, doi: 10.1002/rds19683121154.

[115] F. Sheikh, Y. Gao and T. Kaiser, "A Study of Diffuse Scattering in Massive MIMO Channels at Terahertz Frequencies", IEEE Transactions on Antennas and Propagation, vol. 68, no. 2, pp. 997-1008, 2020. Available: 10.1109/tap.2019.2944536.

[116] N. A. Abbasi et al., "Double Directional Channel Measurements for $\mathrm{THz}$ Communications in an Urban Environment," ICC 2020 - 2020 IEEE International Conference on Communications (ICC), Dublin, Ireland, 2020, pp. 1-6, doi: 10.1109/ICC40277.2020.9148631.
[117] P. Bello, "Characterization of Randomly Time-Variant Linear Channels," in IEEE Transactions on Communications Systems, vol. 11, no. 4, pp. 360-393, December 1963, doi: 10.1109/TCOM.1963.1088793.

[118] Andreas Molisch, Wireless communications: John Wiley \& Sons Ltd., 2011

[119] D. Serghiou et al., "Ultra-Wideband Terahertz Channel Propagation Measurements from 500 to $750 \mathrm{GHz}, 2020$ International Conference on UK-China Emerging Technologies (UCET), Glasgow, United Kingdom, 2020, pp. 1-4, doi: 10.1109/UCET51115.2020.9205476.

[120] Thorlabs, "High-Precision Rotation Stage", https://www.thorlabs.com/newgrouppage9. cfm?objectgroupid $=2464$.

[121] Thorlabs, " $25 \quad \mathrm{~mm}$ Translation Stages", https://www.thorlabs.com/newgrouppage9. cfm?objectgroupid $=10211$

[122] N M Ridler, R G Clarke, C Li and M J Salter, "Strategies for Traceable Submillimeter-wave Vector Network Analyzer Measurements", IEEE Trans Terahertz Science \& Technology, Vol 9, No 4, pp 392-398, July 2019

[123] J. Neu and C. Schmuttenmaer, "Tutorial: An introduction to terahertz time domain spectroscopy (THz-TDS)", Journal of Applied Physics, vol. 124 , no. 23 , p. 231101,2018 . Available: $10.1063 / 1.5047659$.

[124] Michael Hiebel, Fundamentals of Vector Network Analysis. München: Rohde \& Schwarz, 2011.

[125] M. Schmieder et al., "THz Channel Sounding: Design and Validation of a High Performance Channel Sounder at $300 \mathrm{GHz}$," 2020 IEEE Wireless Communications and Networking Conference Workshops (WCNCW), Seoul, Korea (South), 2020, pp. 1-6, doi: 10.1109/WCNCW48565.2020.9124887.

[126] Naftaly, M., Vieweg, N., \& Deninger, A. (2019). Industrial Application of Terahertz Sensing: State of Play. Sensors (Basel, Switzerland), 19(19), 4203. https://doi.org/10.3390/s19194203

[127] W. L. Chan, J. Deibel, and D. M. Mittleman, "Imaging with terahertz radiation," Reports on Progress in Physics, vol. 70, no. 8, pp. 1325-1379, 2007.

[128] M. Klos, R. Bartholdt, J. Klier, J. -. Lampin and R. Beigang, "Photoconductive antennas based on low temperature grown GaAs on silicon substrates for broadband terahertz generation and detection,' 2015 40th International Conference on Infrared, Millimeter, and Terahertz waves (IRMMW-THz), 2015, pp. 1-1, doi: 10.1109/IRMMWTHz.2015.7327851.

[129] Redo-Sanchez, A., Heshmat, B., Aghasi, A. et al. Terahertz time-gated spectral imaging for content extraction through layered structures. Nat Commun 7, 12665 (2016). https://doi.org/10.1038/ncomms12665

[130] G. R. MacCartney, Jr. and T. S. Rappaport, "A flexible millimeter-wave channel sounder with absolute timing," IEEE Journal on Selected Areas in Communications, vol. 35, no. 6, pp. 1402-1418, June 2017.

[131] Zaidi, A., Athley, F., Medbo, J., Gustavsson, U., Durisi, G. and Chen, X., 2018. 5G Physical Layer. Elsevier.

[132] T. W. Crowe, B. Foley, S. Durant, K. Hui, Y. Duan and J. L. Hesler, "VNA frequency extenders to $1.1 \mathrm{THz}, " 2011$ International Conference on Infrared, Millimeter, and Terahertz Waves, Houston, TX, 2011, pp. 1-1, doi: 10.1109/irmmw-THz.2011.6105028

[133] Y. Cui and G. C. Trichopoulos, "A Quasi-Optical Testbed for Wideband THz On-Wafer Measurements," in IEEE Transactions on Terahertz Science and Technology, vol. 9, no. 2, pp. 126-135, March 2019, doi: 10.1109/TTHZ.2019.2894505.

[134] Vadiodes, "THz Vector Network Analyzer Development \& Measurements", https://www.vadiodes.com/VDI/pdf/2010MarNews.pdf

[135] IEEE Std 1785.1-2012, "IEEE Standard for Rectangular Metallic Waveguides and Their Interfaces for Frequencies of $110 \mathrm{GHz}$ and Above- Part 1: Frequency Bands and Waveguide Dimensions".

[136] Vadiodes.com. 2021. Vector Network Analyzer. [online] Available at: ¡https://www.vadiodes.com/en/products/vector-network-analyzer; [Accessed 29 September 2021]

[137] D. Cox, "Delay Doppler characteristics of multipath propagation at 910 $\mathrm{MHz}$ in a suburban mobile radio environment," in IEEE Transactions on Antennas and Propagation, vol. 20, no. 5, pp. 625-635, September 1972, doi: 10.1109/TAP.1972.1140277.

[138] J. D. Parsons, D. A. Demery, and A. M. D. Turkmani, "Sounding techniques for wideband mobile radio channels: a review," IEE Proceedings I - Communications, Speech and Vision, vol. 138, no. 5, pp. 437-446, Oct. 1991.

[139] S. Rey, J. M. Eckhardt, B. Peng, K. Guan and T. Kürner, "Channel sounding techniques for applications in $\mathrm{THz}$ communications: A first correlation based channel sounder for ultra-wideband dynamic channel measurements at $300 \mathrm{GHz}, " 2017$ 9th International 
Congress on Ultra Modern Telecommunications and Control Systems and Workshops (ICUMT), Munich, 2017, pp. 449-453, doi: 10.1109/ICUMT.2017.8255203.

[140] C. U. Bas et al., "Real-Time Millimeter-Wave MIMO Channel Sounder for Dynamic Directional Measurements," in IEEE Transactions on Vehicular Technology, vol. 68, no. 9, pp. 8775-8789, Sept. 2019, doi: 10.1109/TVT.2019.2928341

[141] P. B. Papazian, C. Gentile, K. A. Remley, J. Senic and N. Golmie, "A Radio Channel Sounder for Mobile Millimeter-Wave Communications: System Implementation and Measurement Assessment," in IEEE Transactions on Microwave Theory and Techniques, vol. 64, no. 9, pp. 2924-2932, Sept. 2016, doi: 10.1109/TMTT.2016.2592530.

[142] R. Sun et al., "Design and calibration of a double-directional 60 $\mathrm{GHz}$ channel sounder for multipath component tracking," 2017 11th European Conference on Antennas and Propagation (EUCAP), Paris, France, 2017, pp. 3336-3340, doi: 10.23919/EuCAP.2017.7928270.

[143] A. Glassner, An Introduction to ray tracing. London: Academic Press, 1991.

[144] . He, B. Ai, K. Guan, L. Wang, Z. Zhong and T. Kürner, "The Design and Applications of High-Performance Ray-Tracing Simulation Platform for 5G and Beyond Wireless Communications: A Tutorial," in IEEE Communications Surveys \& Tutorials, vol. 21, no. 1, pp. 10-27, Firstquarter 2019, doi: 10.1109/COMST.2018.2865724.

[145] W. YAMADA, N. KITA, T. SUGIYAMA and T. NOJIMA, "PlaneWave and Vector-Rotation Approximation Technique for Reducing Computational Complexity to Simulate MIMO Propagation Channel Using Ray-Tracing", IEICE Transactions on Communications, vol. 92-, no. 12 , pp. 3850-3860, 2009. Available: 10.1587/transcom.e92.b.3850

[146] Y. Zhao, Y. Hao and C. Parini, "FDTD Characterisation of UWB Indoor Radio Channel Including Frequency Dependent Antenna Directivities," 2006 European Conference on Wireless Technology, Manchester, 2006, pp. 298-301.

[147] N. Cardona, Cooperative radio communications for green smart environments. Gistrup: River Publishers, 2016.

[148] Hao Xu, V. Kukshya and T. S. Rappaport, "Spatial and temporal characteristics of $60-\mathrm{GHz}$ indoor channels," in IEEE Journal on Selected Areas in Communications, vol. 20, no. 3, pp. 620-630, April 2002, doi: 10.1109/49.995521.

[149] Priebe, S., M. Kannicht, M. Jacob, and T. Kürner (2013), Ultra broadband indoor channel measurements and calibrated ray tracing propagation modeling at $\mathrm{THz}$ frequencies, J. Commun. Networking, 15(6), 547-558.

[150] A. A. Saleh and R. A. Valenzuela, "A statistical model for indoor multipath propagation," IEEE Journal on Selected Areas in Communications, vol. 5, no. 2, pp. 128-137, 1987

[151] Q. H. Spencer, B. D. Jeffs, M. A. Jensen and A. L. Swindlehurst, "Modeling the statistical time and angle of arrival characteristics of an indoor multipath channel," in IEEE Journal on Selected Areas in Communications, vol. 18, no. 3, pp. 347-360, March 2000.

[152] Q. Spencer, M. Rice, B. Jeffs and M. Jensen, "A statistical model for angle of arrival in indoor multipath propagation," 1997 IEEE 47th Vehicular Technology Conference. Technology in Motion, Phoenix, AZ, USA, 1997, pp. 1415-1419 vol.3.

[153] J. W. Wallace and M. A. Jensen, "Modeling the indoor MIMO wireless channel," in IEEE Transactions on Antennas and Propagation, vol. 50, no. 5, pp. 591-599, May 2002.

[154] A. Meijerink and A. F. Molisch, "On the Physical Interpretation of the Saleh-Valenzuela Model and the Definition of Its Power Delay Profiles," in IEEE Transactions on Antennas and Propagation, vol. 62, no. 9, pp. 4780-4793, Sept. 2014, doi: 10.1109/TAP.2014.2335812.

[155] Zhao, H., Wei, L., Jarrahi, M. and Pottie, G. (2019). Extending Spatia and Temporal Characterization of Indoor Wireless Channels From 350 to $650 \mathrm{GHz}$. IEEE Transactions on Terahertz Science and Technology, 9(3), pp.243-252.

[156] C. Cheng, S. Sangodoyin and A. Zajić, "THz Cluster-Based Modeling and Propagation Characterization in a Data Center Environment," in IEEE Access, vol. 8, pp. 56544-56558, 2020, doi: 10.1109/ACCESS.2020.2981293

[157] Y. Li, J. Zhang, Z. Ma and Y. Zhang, "Clustering Analysis in the Wireless Propagation Channel with a Variational Gaussian Mixture Model," in IEEE Transactions on Big Data, vol. 6, no. 2, pp. 223232, 1 June 2020, doi: 10.1109/TBDATA.2018.2840696.

[158] S. Priebe, M. Jacob and T. Kuerner, "AoA, AoD and ToA Characteristics of Scattered Multipath Clusters for THz Indoor Channel Modeling," 17th European Wireless 2011 - Sustainable Wireless Technologies, Vienna, Austria, 2011, pp. 1-9.
[159] A. Boulogeorgos, E. Yaqub, M. di Renzo, A. Alexiou, R. Desai and R. Klinkenberg, "Machine Learning: A Catalyst for $\mathrm{THz}$ Wireless Networks", Frontiers in Communications and Networks, vol. 2, 2021. Available: $10.3389 /$ frcmn.2021.704546.

[160] V. Petrov, D. Moltchanov and Y. Koucheryavy, "Applicability assessment of terahertz information showers for next-generation wireless networks," 2016 IEEE International Conference on Communications (ICC), Kuala Lumpur, 2016, pp. 1-7, doi: 10.1109/ICC.2016.7511129.

[161] H. Song et al., "Demonstration of KIOSK data downloading system at $300 \mathrm{GHz}$ based on InP MMICs," 2015 IEEE International Symposium on Radio-Frequency Integration Technology (RFIT), Sendai, 2015, pp. 52-54, doi: 10.1109/RFIT.2015.7377884.

[162] H. Song, H. Hamada and M. Yaita, "Prototype of KIOSK Data Downloading System at $300 \mathrm{GHz}$ : Design, Technical Feasibility, and Results," in IEEE Communications Magazine, vol. 56, no. 6, pp. 130136, June 2018, doi: 10.1109/MCOM.2018.1000939.

[163] D. He et al., "Channel modeling for Kiosk downloading communication system at $300 \mathrm{GHz}$," 2017 11th European Conference on Antennas and Propagation (EUCAP), Paris, 2017, pp. 1331-1335, doi: 10.23919/EuCAP.2017.7928447.

[164] Salhi, M., Kleine-Ostmann, T., Kannicht, M., Priebe, S., Kurner, T. and Schrader, T. (2013). Broadband channel propagation measurements on millimeter and sub-millimeter waves in a desktop download scenario. 2013 Asia-Pacific Microwave Conference Proceedings (APMC).

[165] A. S. Hamza, J. S. Deogun and D. R. Alexander, "Wireless Communication in Data Centers: A Survey," in IEEE Communications Surveys \& Tutorials, vol. 18, no. 3, pp. 1572-1595, thirdquarter 2016, doi: 10.1109/COMST.2016.2521678.

[166] C. Cheng, S. Sangodoyin and A. Zajić, "THz MIMO Channel Characterization for Wireless Data Center-Like Environment," 2019 IEEE International Symposium on Antennas and Propagation and USNCURSI Radio Science Meeting, Atlanta, GA, USA, 2019, pp. 2145-2146, doi: 10.1109/APUSNCURSINRSM.2019.8889030.

[167] C. Cheng, S. Sangodoyin and A. Zajić, "Terahertz MIMO Fading Analysis and Doppler Modeling in a Data Center Environment," 2020 14th European Conference on Antennas and Propagation (EuCAP), Copenhagen, Denmark, 2020, pp. 1-5, doi: 10.23919/EuCAP48036.2020.9135695.

[168] J. M. Eckhardt, T. Doeker, S. Rey and T. Kürner, "Measurements in a Real Data Centre at $300 \mathrm{GHz}$ and Recent Results," 2019 13th European Conference on Antennas and Propagation (EuCAP), Krakow, Poland, 2019, pp. 1-5

[169] B. Peng and T. Kürner, "A stochastic channel model for future wireless THz data centers," 2015 International Symposium on Wireless Communication Systems (ISWCS), Brussels, 2015, pp. 741-745.

[170] S. Mollahasani and E. Onur, "Evaluation of terahertz channel in data centers," NOMS 2016 - 2016 IEEE/IFIP Network Operations and Management Symposium, Istanbul, 2016, pp. 727-730, doi: 10.1109/NOMS.2016.7502886.

[171] S. Abadal, M. Iannazzo, M. Nemirovsky, A. Cabellos-Aparicio, H. Lee and E. Alarcón, "On the Area and Energy Scalability of Wireless Network-on-Chip: A Model-Based Benchmarked Design Space Exploration," in IEEE/ACM Transactions on Networking, vol. 23, no. 5, pp. 1501-1513, Oct. 2015, doi: 10.1109/TNET.2014.2332271.

[172] S. Kim and A. Zajić, "Characterization of 300-GHz Wireless Channel on a Computer Motherboard," in IEEE Transactions on Antennas and Propagation, vol. 64, no. 12, pp. 5411-5423, Dec. 2016, doi: 10.1109/TAP.2016.2620598

[173] S. Abadal, C. Han and J. M. Jornet, "Wave Propagation and Channel Modeling in Chip-Scale Wireless Communications: A Survey From Millimeter-Wave to Terahertz and Optics," in IEEE Access, vol. 8, pp. 278-293, 2020, doi: 10.1109/ACCESS.2019.2961849.

[174] X. Timoneda et al., "Engineer the Channel and Adapt to it: Enabling Wireless Intra-Chip Communication," in IEEE Transactions on Communications, vol. 68, no. 5, pp. 3247-3258, May 2020, doi: 10.1109/TCOMM.2020.2973988.

[175] D. W. Matolak, S. Kaya, and A. Kodi, "Channel modeling for wirelessnetworks-on-chips," IEEE Commun. Mag., vol. 51, no. 6, pp. 180-186,Jun. 2013.

[176] Fricke, A., Achir, M., Le Bars, P. and Kurner, T. (2015). Characterization of transmission scenarios for terahertz intra-device communications. 2015 IEEE-APS Topical Conference on Antennas and Propagation in Wireless Communications (APWC).

[177] Y. Chen and C. Han, "Channel Modeling and Characterization for Wireless Networks-on-Chip Communications in the Millimeter Wave and Terahertz Bands," in IEEE Transactions on Molecular, Biological 
and Multi-Scale Communications, vol. 5, no. 1, pp. 30-43, Oct. 2019, doi: 10.1109/TMBMC.2019.2952863.

[178] J. Nanni et al., "Multi-Path Propagation in On-Chip Optical Wireless Links," in IEEE Photonics Technology Letters, vol. 32, no. 17, pp. 1101-1104, 1 Sept.1, 2020, doi: 10.1109/LPT.2020.3012877.

[179] A.-A. A. Boulogeorgos et al., "Wireless terahertz system architectures for networks beyond 5G," TERRANOVA CONSORTIUM, White Paper 1.0, Jul. 2018.

[180] B. K. Jung, C. Herold, J. M. Eckhardt and T. Kürner, "Link-Level and System-Level Simulation of $300 \mathrm{GHz}$ wireless Backhaul Links," 2020 International Symposium on Antennas and Propagation (ISAP), 2021, pp. 619-620, doi: 10.23919/ISAP47053.2021.9391508.

[181] Kallfass, I., Boes, F., Messinger, T. et al. 64 Gbit/s Transmission over $850 \mathrm{~m}$ Fixed Wireless Link at $240 \mathrm{GHz}$ Carrier Frequency. J Infrared Milli Terahz Waves 36, 221-233 (2015). https://doi.org/10.1007/s10762-014-0140-6

[182] T. Urushihara et al., "60GHz wireless technologies for WiGig/IEEE 802.11ad multi-gigabit systems," 2014 Asia-Pacific Microwave Conference, 2014, pp. 628-630.

[183] S. Ju, Y. Xing, O. Kanhere and T. S. Rappaport, "Millimeter Wave and Sub-Terahertz Spatial Statistical Channel Model for an Indoor Office Building," in IEEE Journal on Selected Areas in Communications, vol. 39, no. 6, pp. 1561-1575, June 2021, doi: 10.1109/JSAC.2021.3071844.

[184] S. Priebe, M. Jacob and T. Kürner, "Calibrated broadband ray tracing for the simulation of wave propagation in $\mathrm{mm}$ and sub-mm wave indoor communication channels," European Wireless 2012; 18th European Wireless Conference 2012, Poznan, Poland, 2012, pp. 1-10.

[185] S. Priebe, M. Jacob, C. Jastrow, T. Kleine-Ostmann, T. Schrader, and T. K" urner, "A Comparison of Indoor Channel Measurements and Ray Tracing Simulations at $300 \mathrm{GHz}$," in 35th International Conference on Infrared, Millimeter and THz Waves (IRMMW-THz), 2 pages (electronic), Rome, September 2010.

[186] A. Glassner, An Introduction to Ray Tracing. Morgan Kaufmann, 1989.

[187] S. Priebe, M. Jacob, C. Jansen, and T. K" urner, "Non-Specular Scattering Modeling for THz Propagation Simulations," in 5th European Conference on Antennas and Propagation (EuCAP), 5 pages (electronic), Rome, April 2011.

[188] Y. Choi, J.-W. Choi, and J. M. Cioffi, "A geometric-statistic channel model for THz indoor communications," J. Infrared Millim. THz. Waves, vol. 34, no. 7, pp. 456-467, 2013.

[189] D. Cahyono, F. Sheikh and T. K. ser, "Deterministic Approach of Indoor Room THz Multipath Channel Model," 2020 8th International Conference on Information and Communication Technology (ICoICT), Yogyakarta, Indonesia, 2020, pp. 1-5, doi: 10.1109/ICoICT49345.2020.9166161.

[190] F. Sheikh, N. Zarifeh and T. Kaiser, "Terahertz band: Channel modelling for short-range wireless communications in the spectral windows," in IET Microwaves, Antennas \& Propagation, vol. 10, no. 13, pp. 1435-1444, 22102016.

[191] S. Priebe, M. Jacob and T. Kürner, "Affection of THz indoor communication links by antenna misalignment," 2012 6th European Conference on Antennas and Propagation (EUCAP), Prague, 2012, pp. 483-487, doi: 10.1109/EuCAP.2012.6206026.

[192] S. Priebe, M. Jacob and T. Kürner, "The impact of antenna directivities on THz indoor channel characteristics," 2012 6th European Conference on Antennas and Propagation (EUCAP), Prague, Czech Republic, 2012, pp. 478-482, doi: 10.1109/EuCAP.2012.6205849.

[193] Priebe, S., Kannicht, M., Jacob, M. and Kurner, T. (2013). Ultra broadband indoor channel measurements and calibrated ray tracing propagation modeling at $\mathrm{THz}$ frequencies. Journal of Communications and Networks, 15(6), pp.547-558.

[194] C. Lin and G. Y. Li, "Indoor Terahertz Communications: How Many Antenna Arrays Are Needed?," in IEEE Transactions on Wireless Communications, vol. 14, no. 6, pp. 3097-3107, June 2015, doi: 10.1109/TWC.2015.2401560.

[195] S. Priebe and T. Kurner, "Stochastic Modeling of $\mathrm{THz}$ Indoor Radio Channels," in IEEE Transactions on Wireless Communications, vol. 12, no. 9, pp. 4445-4455, September 2013, doi: 10.1109/TWC.2013.072313.121581.

[196] Priebe, S., Jastrow, C., Jacob, M., Kleine-Ostmann, T., Schrader, T. and Kurner, T. (2011). Channel and Propagation Measurements at $300 \mathrm{GHz}$. IEEE Transactions on Antennas and Propagation, 59(5), pp.1688-1698.

[197] M. Hall, L. Barclay, and M. Hewitt, "Propagation of Radiowaves,"in Propagation of Radiowaves. Inst. Elect. Eng.: London, U.K.,1996.

[198] R. Piesiewicz et al., "Short-Range Ultra-Broadband Terahertz Communications: Concepts and Perspectives," in IEEE Antennas and
Propagation Magazine, vol. 49, no. 6, pp. 24-39, Dec. 2007, doi: 10.1109/MAP.2007.4455844.

[199] A. Moldovan, P. Karunakaran, I. F. Akyildiz and W. H. Gerstacker, "Coverage and achievable rate analysis for indoor terahertz wireless networks," 2017 IEEE International Conference on Communications (ICC), 2017, pp. 1-7, doi: 10.1109/ICC.2017.7996402.

[200] A. Shafie, N. Yang, Z. Sun and S. Durrani, "Coverage Analysis for 3D Terahertz Communication Systems with Blockage and Directional Antennas," 2020 IEEE International Conference on Communications Workshops (ICC Workshops), Dublin, Ireland, 2020, pp. 1-7, doi: 10.1109/ICCWorkshops49005.2020.9145081.

[201] S. Nie and I. F. Akyildiz, "Three-dimensional dynamic channel modeling and tracking for terahertz band indoor communications," 2017 IEEE 28th Annual International Symposium on Personal, Indoor, and Mobile Radio Communications (PIMRC), Montreal, QC, Canada, 2017, pp. 15, doi: 10.1109/PIMRC.2017.8292607.

[202] J. Wang, C. Wang, J. Huang and H. Wang, "A Novel 3D SpaceTime-Frequency Non-Stationary Channel Model for 6G THz Indoor Communication Systems," 2020 IEEE Wireless Communications and Networking Conference (WCNC), Seoul, Korea (South), 2020, pp. 1-7, doi: 10.1109/WCNC45663.2020.9120570.

[203] S. Ju, Y. Xing, O. Kanhere and T. S. Rappaport, "3-D Statistical Indoor Channel Model for Millimeter-Wave and Sub-Terahertz Bands," GLOBECOM 2020 - 2020 IEEE Global Communications Conference, Taipei, Taiwan, 2020, pp. 1-7, doi: 10.1109/GLOBECOM42002.2020.9322429.

[204] V. Petrov, D. Moltchanov, Y. Koucheryavy and J. M. Jornet, "Capacity and Outage of Terahertz Communications With User MicroMobility and Beam Misalignment," in IEEE Transactions on Vehicular Technology, vol. 69, no. 6, pp. 6822-6827, June 2020, doi: 10.1109/TVT.2020.2988600.

[205] B. Peng and T. Kuerner, "Three-Dimensional Angle of Arrival Estimation in Dynamic Indoor Terahertz Channels Using Forward-Backward Algorithm", IEEE Transactions on Vehicular Technology, pp. 1-1, 2016. Available: $10.1109 /$ tvt.2016.2599488.

[206] B. Peng, K. Guan and T. Kürner, "Cooperative Dynamic Angle of Arrival Estimation Considering Space-Time Correlations for Terahertz Communications," in IEEE Transactions on Wireless Communications, vol. 17, no. 9, pp. 6029-6041, Sept. 2018, doi: 10.1109/TWC.2018.2854565

[207] B. Peng, K. Guan, S. Rey and T. Kürner, "Power-Angular Spectra Correlation Based Two Step Angle of Arrival Estimation for Future Indoor Terahertz Communications," in IEEE Transactions on Antennas and Propagation, vol. 67, no. 11, pp. 7097-7105, Nov. 2019, doi: 10.1109/TAP.2019.2927892.

[208] R. Singh and D. Sicker, "An Analytical Model for Efficient Indoor THz Access Point Deployment," 2020 IEEE Wireless Communications and Networking Conference (WCNC), Seoul, Korea (South), 2020, pp. 1-8, doi: 10.1109/WCNC45663.2020.9120566.

[209] A. Shafie, N. Yang and C. Han, "Multi-Connectivity for Indoor Terahertz Communication with Self and Dynamic Blockage," ICC 2020 - 2020 IEEE International Conference on Communications (ICC), Dublin, Ireland, 2020, pp. 1-7, doi: 10.1109/ICC40277.2020.9148716.

[210] K. Guan et al., "Measurement, Simulation, and Characterization of Train-to-Infrastructure Inside-Station Channel at the Terahertz Band", IEEE Transactions on Terahertz Science and Technology, vol. 9, no. 3, pp. 291-306, 2019. Available: 10.1109/tthz.2019.2909975.

[211] K. Guan et al., "Channel Characterization for Intra-Wagon Communication at 60 and $300 \mathrm{GHz}$ Bands," in IEEE Transactions on Vehicular Technology, vol. 68, no. 6, pp. 5193-5207, June 2019, doi: 10.1109/TVT.2019.2907606.

[212] X. Yuan, Y. -J. A. Zhang, Y. Shi, W. Yan and H. Liu, "ReconfigurableIntelligent-Surface Empowered Wireless Communications: Challenges and Opportunities," in IEEE Wireless Communications, vol. 28, no. 2, pp. 136-143, April 2021, doi: 10.1109/MWC.001.2000256.

[213] K. Guan et al., "Channel Sounding and Ray Tracing for Intrawagon Scenario at mmWave and Sub-mmWave Bands," in IEEE Transactions on Antennas and Propagation, vol. 69, no. 2, pp. 1007-1019, Feb. 2021, doi: 10.1109/TAP.2020.3016399.

[214] K. Guan et al., "Channel Characterization and Capacity Analysis for THz Communication Enabled Smart Rail Mobility," in IEEE Transactions on Vehicular Technology, vol. 70, no. 5, pp. 4065-4080, May 2021, doi: 10.1109/TVT.2021.3071242.

[215] N. Saeed, A. Elzanaty, H. Almorad, H. Dahrouj, T. Y. Al-Naffouri and M. -S. Alouini, "CubeSat Communications: Recent Advances and Future Challenges," in IEEE Communications Surveys \& Tu- 
torials, vol. 22, no. 3, pp. 1839-1862, thirdquarter 2020, doi: 10.1109/COMST.2020.2990499.

[216] R. Radhakrishnan, W. W. Edmonson, F. Afghah, R. M. RodriguezOsorio, F. Pinto and S. C. Burleigh, "Survey of Inter-Satellite Communication for Small Satellite Systems: Physical Layer to Network Layer View," in IEEE Communications Surveys \& Tutorials, vol. 18, no. 4, pp. 2442-2473, Fourthquarter 2016, doi: 10.1109/COMST.2016.2564990.

[217] A. H. Hashim, F. D. Mahad, S. M. Idrus and A. S. M. Supa'at, "Modeling and performance study of inter-satellite optical wireless communication system," International Conference On Photonics 2010, 2010, pp. 1-4, doi: 10.1109/ICP.2010.5604379.

[218] Y. Li and Y. Chen, "Propagation Modeling and Analysis for Terahertz Inter-satellite Communications Using FDTD Methods," 2021 IEEE International Conference on Communications Workshops (ICC Workshops), 2021, pp. 1-6, doi: 10.1109/ICCWorkshops50388.2021.9473712.

[219] S.-W. Dong, Z.-b. Zhu, and Y. Wang, "Advances of terahertz research and terahertz satellite communications," in 2011 IEEE Int. Conf. Electron. Commun. Control, 2011, pp. 4122-4125.

[220] T. Nagatsuma and A. Kasamatsu, "Terahertz communications for space applications," in 2018 Asia-Pacific Microwave Conference (APMC). IEEE, 2018, pp. 73-75.

[221] Y.-M. Ding, S. Gao, X. Shi, and H. Wu, "Analysis of inter-satellite terahertz communication link," in 3rd Int. Conf. Wireless Commun. Sensor Netw., 2016, pp. 180-183.

[222] S. Nie and I. F. Akyildiz, "Channel Modeling and Analysis of Inter-Small-Satellite Links in Terahertz Band Space Networks," in IEEE Transactions on Communications, doi: 10.1109/TCOMM.2021.3113942.

[223] Y. Liu, L. Tan and J. Ma, ’Doppler effect performance for multiple hops optical connections in LEO satellite constellations", Optik International Journal for Light and Electron Optics, vol. 124, no. 18, pp. 3453-3456, 2013. Available: 10.1016/j.ijleo.2012.10.022.

[224] Min Wang, Jinqing Li, Y. Jiang and Xiaoqiang Di, "Stochastic performance analysis for LEO inter-satellite link based on finite-state Markov chain modeling," 2015 4th International Conference on Computer Science and Network Technology (ICCSNT), 2015, pp. 1230-1235, doi: 10.1109/ICCSNT.2015.7490954.

[225] S. Pradhan, P. K. Sahu, R. K. Giri and B. Patnaik, "Inter-satellite optical wireless communication system design using diversity techniques,' 2015 International Conference on Microwave, Optical and Communication Engineering (ICMOCE), 2015, pp. 250-253, doi: 10.1109/ICMOCE.2015.7489738.

[226] "Terahertz Communication for Vehicular Networks," in IEEE Transactions on Vehicular Technology, vol. 66, no. 7, pp. 5617-5625, July 2017, doi: 10.1109/TVT.2017.2712878.

[227]@articleNoorARahim20206GFV, title=6G for Vehicle-to-Everything (V2X) Communications: Enabling Technologies, Challenges, and Opportunities, author=Md. Noor-A.-Rahim and Zilong Liu and Haeyoung Lee and M. O. Khyam and Jianhua $\mathrm{He}$ and D. Pesch and K. Moessner and W. Saad and H. Poor, journal=ArXiv, year=2020, volume $=\mathrm{abs} / 2012.07753$

[228] J. Choi, V. Va, N. Gonzalez-Prelcic, R. Daniels, C. R. Bhat and R. W. Heath, "Millimeter-Wave Vehicular Communication to Support Massive Automotive Sensing," in IEEE Communications Magazine, vol. 54, no. 12, pp. 160-167, December 2016, doi: 10.1109/MCOM.2016.1600071CM.

[229] V. Petrov, D. Moltchanov, S. Andreev and R. W. Heath, "Analysis of Intelligent Vehicular Relaying in Urban 5G+ MillimeterWave Cellular Deployments," 2019 IEEE Global Communications Conference (GLOBECOM), 2019, pp. 1-6, doi: 10.1109/GLOBECOM38437.2019.9013636

[230] V. Petrov, J. Kokkoniemi, D. Moltchanov, J. Lehtomäki, M. Juntti and Y. Koucheryavy, "The Impact of Interference From the Side Lanes on mmWave/THz Band V2V Communication Systems With Directional Antennas," in IEEE Transactions on Vehicular Technology, vol. 67, no. 6, pp. 5028-5041, June 2018, doi: 10.1109/TVT.2018.2799564.

[231] J. M. Eckhardt, V. Petrov, D. Moltchanov, Y. Koucheryavy and T. Kürner, "Channel Measurements and Modeling for Low-Terahertz Band Vehicular Communications," in IEEE Journal on Selected Areas in Communications, vol. 39, no. 6, pp. 1590-1603, June 2021, doi 10.1109/JSAC.2021.3071843.

[232] V. Petrov, J. M. Eckhardt, D. Moltchanov, Y. Koucheryavy and T. Kurner, "Measurements of Reflection and Penetration Losses in Low Terahertz Band Vehicular Communications," 2020 14th European Con- ference on Antennas and Propagation (EuCAP), 2020, pp. 1-5, doi: 10.23919/EuCAP48036.2020.9135389.

[233] S. A. Busari et al., "Generalized Hybrid Beamforming for Vehicular Connectivity Using THz Massive MIMO," in IEEE Transactions on Vehicular Technology, vol. 68, no. 9, pp. 8372-8383, Sept. 2019, doi: 10.1109/TVT.2019.2921563.

[234] Yi, H., Guan, K., He, D., Ai, B., Dou, J. and Kim, J. (2019) Characterization for the Vehicle-to-Infrastructure Channel in Urban and Highway Scenarios at the Terahertz Band. IEEE Access, 7, pp.166984166996.

[235] Y. Chen and C. Han, "Time-Varying Channel Modeling for Low-Terahertz Urban Vehicle-to-Infrastructure Communications," 2019 IEEE Global Communications Conference (GLOBECOM), Waikoloa, HI, USA, 2019, pp. 1-6, doi: 10.1109/GLOBECOM38437.2019.9013865.

[236] C. Han and I. F. Akyildiz, "Three-Dimensional End-to-End Modeling and Analysis for Graphene-Enabled Terahertz Band Communications," in IEEE Transactions on Vehicular Technology, vol. 66, no. 7, pp. 5626-5634, July 2017, doi: 10.1109/TVT.2016.2614335.

[237] M. Haus, M. Waqas, A. Y. Ding, Y. Li, S. Tarkoma and J. Ott, "Security and Privacy in Device-to-Device (D2D) Communication: A Review," in IEEE Communications Surveys \& Tutorials, vol. 19, no. 2, pp. 10541079, Secondquarter 2017, doi: 10.1109/COMST.2017.2649687.

[238] P. Gandotra and R. Jha, "Device-to-Device Communication in Cellular Networks: A Survey", Journal of Network and Computer Applications, vol. 71, pp. 99-117, 2016. Available: 10.1016/j.jnca.2016.06.004.

[239] N. A. Abbasi et al., "Double-Directional Channel Measurements for Urban THz Communications on a Linear Route," 2021 IEEE International Conference on Communications Workshops (ICC Workshops), 2021, pp. 1-6, doi: 10.1109/ICCWorkshops50388.2021.9473566.

[240] S. Kim and A. Zajić, "Statistical Modeling and Simulation of ShortRange Device-to-Device Communication Channels at Sub-THz Frequencies," in IEEE Transactions on Wireless Communications, vol. 15, no. 9, pp. 6423-6433, Sept. 2016, doi: 10.1109/TWC.2016.2585103.

[241] J. Jornet and I. Akyildiz, "Channel Modeling and Capacity Analysis for Electromagnetic Wireless Nanonetworks in the Terahertz Band", IEEE Transactions on Wireless Communications, vol. 10, no. 10, pp. 3211-3221, 2011. Available: 10.1109/twc.2011.081011.100545.

[242] R. M. Goody and Y. L. Yung, Atmospheric Radiation: Theoretical Basis, 2nd edition. Oxford University Press, 1989.

[243] H. Elayan, R. Shubair, J. Jornet and P. Johari, "Terahertz Channel Model and Link Budget Analysis for Intrabody Nanoscale Communication", IEEE Transactions on NanoBioscience, vol. 16, no. 6, pp. 491-503, 2017. Available: 10.1109/tnb.2017.2718967.

[244] K. Yang, A. Pellegrini, M. Munoz, A. Brizzi, A. Alomainy and Y. Hao, "Numerical Analysis and Characterization of $\mathrm{THz}$ Propagation Channel for Body-Centric Nano-Communications", IEEE Transactions on Terahertz Science and Technology, vol. 5, no. 3, pp. 419-426, 2015. Available: 10.1109/tthz.2015.2419823.

[245] Q. Abbasi, H. El Sallabi, N. Chopra, K. Yang, K. Qaraqe and A. Alomainy, "Terahertz Channel Characterization Inside the Human Skin for Nano-Scale Body-Centric Networks", IEEE Transactions on Terahertz Science and Technology, vol. 6, no. 3, pp. 427-434, 2016 Available: 10.1109/tthz.2016.2542213 [Accessed 8 April 2020].

[246] H. Elayan, C. Stefanini, R. M. Shubair and J. M. Jornet, "End-to-End Noise Model for Intra-Body Terahertz Nanoscale Communication," in IEEE Transactions on NanoBioscience, vol. 17, no. 4, pp. 464-473, Oct. 2018, doi: 10.1109/TNB.2018.2869124.

[247] A. M. Vegni and V. Loscrí, "Analysis of the Chirality Effects on the Capacity of Wireless Communication Systems in the THz Band," in IEEE Transactions on Wireless Communications, vol. 16, no. 12, pp. 7848-7858, Dec. 2017, doi: 10.1109/TWC.2017.2754259.

[248] E. Plum, M. Ren, V. Fedotov, J. Xu, and N. Zheludev. (Apr. 2012). Giant Linear and Nonlinear Optical Activity in Metamaterials. [Online]. Available: http://eprints.soton.ac.uk/379293/

[249] C. Han, A. Bicen and I. Akyildiz, "Multi-Ray Channel Modeling and Wideband Characterization for Wireless Communications in the Terahertz Band", IEEE Transactions on Wireless Communications, vol. 14, no. 5, pp. 2402-2412, 2015. Available: 10.1109/twc.2014.2386335.

[250] A. Moldovan, M. A. Ruder, I. F. Akyildiz and W. H. Gerstacker, 'LOS and NLOS channel modeling for terahertz wireless communication with scattered rays," 2014 IEEE Globecom Workshops (GC Wkshps), Austin, TX, 2014, pp. 388-392.

[251] 65. K. Tsujimura, K. Umebayashi, J. Kokkoniemi, J. Lehtomäki and Y. Suzuki, "A Causal Channel Model for the Terahertz Band," in IEEE Transactions on Terahertz Science and Technology, vol. 8, no. 1, pp. 52-62, Jan. 2018. 
[252] J. Wang, C. -X. Wang, J. Huang, H. Wang and X. Gao, "A General 3D Space-Time-Frequency Non-Stationary $\mathrm{THz}$ Channel Model for 6G Ultra-Massive MIMO Wireless Communication Systems," in IEEE Journal on Selected Areas in Communications, vol. 39, no. 6, pp. 15761589, June 2021, doi: 10.1109/JSAC.2021.3071850.

[253] S.K. Choudhury, S. Chinchanikar, 1.3 Finish Machining of Hardened Steel, Editor(s): MSJ Hashmi, Comprehensive Materials Finishing, Elsevier, 2017, Pages 47-92, ISBN 9780128032497, https://doi.org/10.1016/B978-0-12-803581-8.09149-9. (http://www.sciencedirect.com/science/article/pii/B9780128035818091499)

[254] A. Bystrov, E. Hoare, M. Gashinova, T. Tran and M. Cherniakov, "Low terahertz signal backscattering from rough surfaces", Proceedings of the 16th European Radar Conference, 2019.

[255] A. Bystrov, E. Hoare, M. Gashinova, M. Cherniakov and T. Tran, "Experimental Study of Rough Surface Backscattering for Low Terahertz Automotive Radar", 2019 20th International Radar Symposium (IRS), 2019. Available: 10.23919/irs.2019.8768126.

[256] A. Jagannathan, A. Gatesman and R. Giles, "Characterization of roughness parameters of metallic surfaces using terahertz reflection spectra", Optics Letters, vol. 34, no. 13, p. 1927, 2009. Available: 10.1364/ol.34.001927.

[257] B. Yang, M. Kirley and J. Booske, ”Theoretical and Empirical Evaluation of Surface Roughness Effects on Conductivity in the Terahertz Regime", IEEE Transactions on Terahertz Science and Technology, vol. 4, no. 3, pp. 368-375, 2014. Available: 10.1109/tthz.2014.2310121.

[258] M. Herrmann, C. Wiegand, J. Jonuscheit and R. Beigang, "The influence of surface roughness on THz reflection measurements," 2009 34th International Conference on Infrared, Millimeter, and Terahertz Waves, Busan, 2009, pp. 1-2.

[259] Kurner, T., Piesiewicz, R., Koch, M. and Schoebel, J. (2007). Propagation Models, Measurements and Simulations for Wireless Communication Systems beyond $100 \mathrm{GHz} .2007$ International Conference on Electromagnetics in Advanced Applications.

[260] Alicona Imaging InfiniteFocus, AliconaImaging $\mathrm{GmbH}$ (www.alicona.com)

[261] J. Ma, R. Shrestha, L. Moeller and D. Mittleman, "Channel Characteristics for Terahertz Wireless Communications", 2018 43rd International Conference on Infrared, Millimeter, and Terahertz Waves (IRMMWTHz), 2018. Available: 10.1109/irmmw-thz.2018.8509931 [Accessed 22 March 2020].

[262] F. Sheikh, Q. H. Abbasi and T. Kaiser, "On Channels with Composite Rough Surfaces at Terahertz Frequencies," 2019 13th European Conference on Antennas and Propagation (EuCAP), Krakow, Poland, 2019, pp. 1-5.

[263] Mandehgar, M., Yang, Y. \& Grischkowsky, D. Experimental confirmation and physical understanding of ultra-high bit rate impulse radio in the $\mathrm{THz}$ digital communication channels of the atmosphere. Journal of Optics16,094004, https://doi.org/10.1088/20408978/16/9/094004 (2014).

[264] McMillan R.W. (2006) TERAHERTZ IMAGING, MILLIMETERWAVE RADAR. In: Byrnes J., Ostheimer G. (eds) Advances in Sensing with Security Applications. NATO Security Through Science Series, vol 2. Springer, Dordrecht. https://doi.org/10.1007/1-4020-4295-7_11

[265] K. Strecker, S. Ekin and J. O'Hara, "Compensating Atmospheric Channel Dispersion for Terahertz Wireless Communication", Scientific Reports, vol. 10, no. 1, 2020. Available: 10.1038/s41598-020-62692-7 [Accessed 16 April 2020].

[266] Guidelines for Limiting Exposure to Electromagnetic Fields (100 $\mathrm{kHz}$ to $300 \mathrm{GHz}$ ). Health Phys. 2020 May;118(5):483-524. doi: 10.1097/HP.000000000001210. PMID: 32167495

[267] T. Wu, T. S. Rappaport, and C. M. Collins, "Safe for generations to come: Considerations of safety for millimeter waves in wireless communications," IEEE Microwave Magazine, vol. 16, no. 2, pp. 65-84, Mar. 2015.

[268] C. Cho et al., "Characterizing Ionizing Radiation Exposure after T-Cell Depleted Allogeneic Hematopoietic Cell Transplantation," Biology of Blood and Marrow Transplantation, vol. 24, issue 3, Supplement, pp. S252-S253, Mar. 2018.

[269] T. Wu, T. S. Rappaport, and C. M. Collins, "The human body and millimeter-wave wireless communication systems: Interactions and implications," in 2015 IEEE International Conference on Communications (ICC), June 2015, pp. 2423-2429.

[270] I. Echchgadda et al., "Terahertz Radiation: A Non-contact Tool for the Selective Stimulation of Biological Responses in Human Cells," in IEEE Transactions on Terahertz Science and Technology, vol. 6, no. 1, pp. 54-68, Jan. 2016, doi: 10.1109/TTHZ.2015.2504782.
[271] Cherkasova, O.P., Serdyukov, D.S., Ratushnyak, A.S. et al. Effects of Terahertz Radiation on Living Cells: a Review. Opt. Spectrosc. 128 855-866 (2020). https://doi.org/10.1134/S0030400X20060041

[272] I. F. Akyildiz, C. Han and S. Nie, "Combating the Distance Problem in the Millimeter Wave and Terahertz Frequency Bands," in IEEE Communications Magazine, vol. 56, no. 6, pp. 102-108, June 2018 , doi: 10.1109/MCOM.2018.1700928.

[273] E. Björnson, J. Hoydis and L. Sanguinetti, "Massive MIMO Has Unlimited Capacity," in IEEE Transactions on Wireless Communications, vol. 17, no. 1, pp. 574-590, Jan. 2018, doi: 10.1109/TWC.2017.2768423.

[274] Ian F. Akyildiz, Josep Miquel Jornet, Realizing Ultra-Massive MIMO $(1024 \times 1024)$ communication in the $(0.06-10)$ Terahertz band, Nano Communication Networks, Volume 8, 2016, Pages 4654, ISSN 1878-7789, https://doi.org/10.1016/j.nancom.2016.02.001. (http://www.sciencedirect.com/science/article/pii/S1878778916000107)

[275] K. Yao, Y. Liu, Plasmonic metamaterials, Nanotechnol. Rev. 3 (2) (2014) 177-210

[276] A. Boltasseva, H.A. Atwater, Low-loss plasmonic metamaterials, Science 331 (6015) (2011) 290-291.

[277] A. Faisal, H. Sarieddeen, H. Dahrouj, T. Y. Al-Naffouri and M. -S. Alouini, "Ultramassive MIMO Systems at Terahertz Bands: Prospects and Challenges," in IEEE Vehicular Technology Magazine, vol. 15, no. 4, pp. 33-42, Dec. 2020, doi: 10.1109/MVT.2020.3022998.

[278] C. Han, J. M. Jornet and I. Akyildiz, "Ultra-Massive MIMO Channel Modeling for Graphene-Enabled Terahertz-Band Communications," 2018 IEEE 87th Vehicular Technology Conference (VTC Spring), Porto, 2018, pp. 1-5, doi: 10.1109/VTCSpring.2018.8417893.

[279] S. Nie, J. M. Jornet and I. F. Akyildiz, 'Intelligent Environments Based on Ultra-massive Mimo Platforms for Wireless Communication in Millimeter Wave and Terahertz Bands," ICASSP 2019 - 2019 IEEE International Conference on Acoustics, Speech and Signal Processing (ICASSP), Brighton, United Kingdom, 2019, pp. 7849-7853, doi: 10.1109/ICASSP.2019.8683394

[280] P. F. Driessen and G. Foschini, "On the capacity formula for multiple input-multiple output wireless channels: A geometric interpretation," IEEE Trans. Commun., vol. 47, pp. 173-176, Feb. 1999.

[281] Y. Chen and C. Han, "Deep CNN-Based Spherical-Wave Channel Estimation for Terahertz Ultra-Massive MIMO Systems," GLOBECOM 2020 - 2020 IEEE Global Communications Conference, Taipei, Taiwan, 2020, pp. 1-6, doi: 10.1109/GLOBECOM42002.2020.9322174.

[282] Y. Miao, J. Takada, K. Saito, K. Haneda, A. A. Glazunov and Y. Gong, "Comparison of Plane Wave and Spherical Vector Wave Channel Modeling for Characterizing Non-Specular Rough-Surface Wave Scattering," in IEEE Antennas and Wireless Propagation Letters, vol. 17 no. 10, pp. 1847-1851, Oct. 2018, doi: 10.1109/LAWP.2018.2868108,

[283] Y. Chen, L. Yan and C. Han, "Millidegree-Level Direction-of-Arrival (DoA) Estimation and Tracking for Terahertz Wireless Communications," 2020 17th Annual IEEE International Conference on Sensing, Communication, and Networking (SECON), Como, Italy, 2020, pp. 1-9, doi: 10.1109/SECON48991.2020.9158439.

[284] ITU-R Rec. P.1238-10. Propagation Data and Prediction Methods for the Planning of Indoor Radiocommunication Systems and Radio Local Area Networks in the Frequency Range $300 \mathrm{MHz}$ to $450 \mathrm{GHz}$; ITU Radiocommunication Bureau: Geneva, Switzerland, 2019

[285] ITU-R Recommendations, ITU-R P. 1238-7 "Propagation data and prediction methods for the planning of indoor radiocommunication systems and radio local area networks in the frequency range $900 \mathrm{MHz}$ to $100 \mathrm{GHz} " 2012$.

[286] G. Gougeon, Y. Corre and M. Z. Aslam, "Ray-based Deterministic Channel Modelling for sub-THz Band," 2019 IEEE 30th International Symposium on Personal, Indoor and Mobile Radio Communications (PIMRC Workshops), Istanbul, Turkey, 2019, pp. 1-6.

[287] S.K. Yee, A. Sayegh, A. Kazemipour, and M.Z.M. Jenu. Design and calibration of a wideband TEM-cell for material characterization, in 2012 Asia-Pacific Symposium on Electromagnetic Compatibility, Singapore, 2012, pp. 749-752.

[288] Y. Kato, M. Horibe, M. Ameya, S. Kurokawa, and Y. Shimada, New uncertainty analysis for permittivity measurements using the transmission/reflection method, IEEE Trans. Instrum. Meas. 64 (2015), no. $6,1748-1753$.

[289] B. Komiyama, M. Kiyokawa, and T. Matsui, Open resonator for precision dielectric measurements in the $100 \mathrm{GHz}$ band, IEEE Trans. Microwave Theory Tech. 39 (1991), no. 10, 1792-1796.

[290] B. Ai et al., "On Indoor Millimeter Wave Massive MIMO Channels: Measurement and Simulation," in IEEE Journal on Selected Areas 
in Communications, vol. 35, no. 7, pp. 1678-1690, July 2017, doi: 10.1109/JSAC.2017.2698780.

[291] Kazemipour, A., Wollensack, M., Hoffmann, J. et al. Analytical Uncertainty Evaluation of Material Parameter Measurements at $\mathrm{THz}$ Frequencies. J Infrared Milli Terahz Waves 41, 1199-1217 (2020). https://doi.org/10.1007/s10762-020-00723-0

[292] . Jiao, J. J. Hall, and Y. T. Morton, "Automatic equatorial gpsamplitude scintillation detection using a machine learning algorithm,'IEEE Transactions on Aerospace and Electronic Systems, vol. 53, no. 1,pp. 405-418, 2017.

[293] T. Imai, K. Kitao and M. Inomata, "Radio Propagation Prediction Model Using Convolutional Neural Networks by Deep Learning," 2019 13th European Conference on Antennas and Propagation (EuCAP), Krakow, Poland, 2019, pp. 1-5.

[294] T. Hayashi, T. Nagao and S. Ito, "A study on the variety and size of input data for radio propagation prediction using a deep neural network," 2020 14th European Conference on Antennas and Propagation (EuCAP), Copenhagen, Denmark, 2020, pp. 1-5, doi: 10.23919/EuCAP48036.2020.9135876.

[295] B. Peng, Q. Jiao and T. Kürner, "Angle of arrival estimation in dynamic indoor $\mathrm{THz}$ channels with Bayesian filter and reinforcement learning," 2016 24th European Signal Processing Conference (EUSIPCO), Budapest, Hungary, 2016, pp. 1975-1979, doi: 10.1109/EUSIPCO.2016.7760594.

[296] J. Tak, A. Kantemur, Y. Sharma, and H. Xin, "A 3-d-printedwbandslotted waveguide array antenna optimized using machine learning,'IEEE Antennas and Wireless Propagation Letters, vol. 17, no. 11, pp.2008-2012, 2018.

[297] Jaewook Lee and Daewon Lee, "An improved cluster labeling method for support vector clustering," in IEEE Transactions on Pattern Analysis and Machine Intelligence, vol. 27, no. 3, pp. 461-464, March 2005, doi: 10.1109/TPAMI.2005.47.

[298] Ying Wang, S. Safavi-Naeini and S. K. Chaudhuri, "A hybrid technique based on combining ray tracing and FDTD methods for site-specific modeling of indoor radio wave propagation," in IEEE Transactions on Antennas and Propagation, vol. 48, no. 5, pp. 743-754, May 2000.

[299] K. Nallappan, H. Guerboukha, C. Nerguizian and M. Skorobogatiy, "Live Streaming of Uncompressed HD and 4K Videos Using Terahertz Wireless Links," in IEEE Access, vol. 6, pp. 58030-58042, 2018, doi: 10.1109/ACCESS.2018.2873986. 